\title{
Equilibrium configurations of epitaxially strained crystalline films: existence and regularity results
}

\author{
I. Fonseca* N. Fusco, G. Leoni
}

January 3, 2007

\begin{abstract}
Strained epitaxial films grown on a relatively thick substrate are considered in the context of plane linear elasticity. The total free energy of the system is assumed to be the sum of the energy of the free surface of the film and the strain energy. Because of the lattice mismatch between film and substrate, flat configurations are in general energetically unfavourable and a corrugated or islanded morphology is the preferred growth mode of the strained film. After specifying the functional setup where the existence problem can be properly framed, a study of the qualitative properties of the solutions is undertaken. New regularity results for volume constrained local minimizers of the total free energy are established, leading, as a byproduct, to a rigorous proof of the zero contact-angle condition between islands and wetting layers.
\end{abstract}

\section{Introduction}

In this paper we study from the variational point of view a mathematical model for the epitaxial deposition of a film onto a relatively thick substrate in the case where there is a mismatch between the lattice parameters of the two crystalline solids.

At the interface between the film and the substrate two opposing mechanisms compete to determine the resulting structure. Ideally the minimum energy configuration of the bulk material occurs at the stress-free state for each solid, however when the lattice parameters of the two materials differ, complete relaxation to bulk equilibrium would result in a crystalline structure that would be discontinuous at the interface. As this is forbidden due to the constraint of epitaxy, a mismatch strain in the film arises during deposition.

The presence of such a strain renders a flat layer of the film morphologically unstable or metastable, after a critical value of the thickness is reached. This is explained as the effect of the competition between the surface energy and the bulk energy: To release some of the elastic energy due to the strain, the atoms on the free surface of the film tend to rearrange into a more favorable configuration. In turn, such a migration of atoms has an energetic prize in terms of surface tension and the resulting configuration has lower total energy only if the thickness of the film is large enough. We refer to [16] for a detailed mathematical discussion of this threshold effect.

Typically, after entering the instability regime, the film surface becomes wavy or the material agglomerates into clusters or isolated islands on the substrate surface. Island formation in systems such as In-GaAs/GaAs or SiGe/Si turns out to be useful in the fabrication of modern semiconductor

\footnotetext{
*Department of Mathematical Sciences, Carnegie-Mellon University, Pittsburgh, PA, U.S.A., fonseca@andrew.cmu.edu

†Dipartimento di Matematica e Applicazioni "R. Caccioppoli", Università degli Studi di Napoli "Federico II" , Napoli, Italy, n.fusco@unina.it

$¥$ Department of Mathematical Sciences, Carnegie-Mellon University, Pittsburgh, PA, U.S.A., giovanni@andrew.cmu.edu

$\S$ S.I.S.S.A., Trieste, Italy, morini@sissa.it
} 
electronic and optoelectronic devices such as quantum dots laser. Let us mention here that there are two different modes of island growth: In the first one, known as the Stranski-Krastanow (SK) mode, the islands are separated by a thin wetting layer, while in the second one, the Volmer-Weber (VW) mode, no wetting occurs and the substrate is exposed between islands.

In the literature several atomistic and continuum theories for the growth of epitaxially strained solid films are available. Here we work in the context of continuum mechanics and we essentially follow the variational approach contained in [26] (see also [28], [20], and the references contained therein).

We now describe the model considered in this paper. Both the film and the substrate are modeled as linearly elastic solids. To keep the geometry as simple as possible we restrict attention to an epitaxial layer (with variable thickness $h$ ) grown on a flat semi-infinite substrate. We further restrict attention to two-dimensional morphologies which correspond to three-dimensional configurations with planar symmetry.

We assume that the material occupies the infinite strip

$$
\Omega:=\{\mathbf{x}=(x, y): a<x<b, y<h(x)\}
$$

where $h:[a, b] \rightarrow[0, \infty)$. Thus the graph of $h$ represents the free profile of the film, the open set $\Omega^{+}=\Omega \cap\{y>0\}$ is the reference configuration of the film, and the line $y=0$ corresponds to the film/substrate interface. We work within the theory of small deformations, so that

$$
\mathbf{E}(\mathbf{u}):=\frac{1}{2}\left(\nabla \mathbf{u}+\nabla \mathbf{u}^{T}\right)
$$

represents the strain, with $\mathbf{u}: \Omega \rightarrow \mathbb{R}^{2}$ the planar displacement. The displacement is measured from a configuration of the layer in which the lattices of the film and the layer are perfectly matched; this configuration, in which $\mathbf{E} \equiv \mathbf{0}$, will not correspond to a minimum energy state of the film, which we assume to occur at a strain $\mathbf{E}_{0}=\mathbf{E}_{0}(y)$. We assume that this mismatch strain has the specific form

$$
\mathbf{E}_{0}(y)= \begin{cases}e_{0} \mathbf{i} \otimes \mathbf{i} & \text { if } y \geq 0 \\ 0 & \text { if } y<0\end{cases}
$$

with $e_{0}>0$ and $\mathbf{i}$ the unit vector along the $x$ direction (throughout all the paper $\{\mathbf{i}, \mathbf{j}\}$ will denote the canonical basis of $\mathbb{R}^{2}$ ).

In our setting the film and the substrate have similar material properties, and so they share the same homogeneous elasticity tensor $C$. Hence, bearing in mind the mismatch, the elastic energy per unit area is given by $W\left(\mathbf{E}-\mathbf{E}_{0}(y)\right)$, where

$$
W(\mathbf{E}):=\frac{1}{2} \mathbf{E} \cdot C[\mathbf{E}]
$$

with $C$ a positive definite fourth-order tensor, that is,

$$
\frac{1}{2} \mathbf{E} \cdot C[\mathbf{E}]>0
$$

for all symmetric matrices $\mathbf{E} \neq 0$.

In the sharp interface model the interfacial energy density $\varphi_{0}$ has a step discontinuity at $y=0$ : It is $\gamma_{\text {film }}$ if the film has positive thickness and $\gamma_{\text {sub }}$ if the substrate is exposed, precisely

$$
\varphi_{0}(y):= \begin{cases}\gamma_{\text {film }} & \text { if } y>0 \\ \gamma_{\text {sub }} & \text { if } y=0\end{cases}
$$

Hence the total energy of the system is given by

$$
F(\mathbf{u}, \Omega):=\int_{\Omega} W\left(\mathbf{E}(\mathbf{u})(\mathbf{x})-\mathbf{E}_{0}(y)\right) d \mathbf{x}+\int_{\Gamma} \varphi_{0}(y) d \mathcal{H}^{1}(\mathbf{x}),
$$


where $\Gamma$ represents the free surface of the film, that is,

$$
\Gamma:=\partial \Omega \cap((a, b) \times \mathbb{R}) .
$$

As we already mentioned above the sharp interface model is difficult to be implemented numerically. Thus in the literature it is customary to replace it with a boundary-layer model, where the discontinuous transition is regularized over a thin transition region of width $\delta$ ("smearing parameter").

In this paper, following the work of Spencer [26] (see also the references therein), for $\delta>0$ we consider the regularized mismatch strain

$$
\mathbf{E}_{\delta}(y):=\frac{1}{2} e_{0}\left(1+f\left(\frac{y}{\delta}\right)\right) \mathbf{i} \otimes \mathbf{i}, \quad y \in \mathbb{R},
$$

and the regularized surface energy density

$$
\varphi_{\delta}(y):=\gamma_{\mathrm{sub}}+\left(\gamma_{\mathrm{film}}-\gamma_{\mathrm{sub}}\right) f\left(\frac{y}{\delta}\right), \quad y \geq 0,
$$

where $f$ is a smooth increasing function such that

$$
f(0)=0, \quad \lim _{y \rightarrow-\infty} f(y)=-1, \quad \lim _{y \rightarrow \infty} f(y)=1 .
$$

Thus the regularized total energy of the system becomes

$$
F_{\delta}(\mathbf{u}, \Omega):=\int_{\Omega} W\left(\mathbf{E}(\mathbf{u})(\mathbf{x})-\mathbf{E}_{\delta}(y)\right) d \mathbf{x}+\int_{\Gamma} \varphi_{\delta}(y) d \mathcal{H}^{1}(\mathbf{x}) .
$$

The paper is divided into two parts. In the first part we study the asymptotics as $\delta \rightarrow 0^{+}$of the regularized problem and we show that the limiting functional is given by a suitable relaxed version of the sharp interface model energy (1.6) (see Theorem 2.9). We consider here both regimes $\gamma_{\text {film }} \geq \gamma_{\text {sub }}$ and $\gamma_{\text {film }}<\gamma_{\text {sub }}$. It is interesting to note that in the latter regime the relaxed surface energy density is no longer discontinuous and in fact it is constantly equal to $\gamma_{\text {film. }}$. From the physical point of view this may be seen as evidence of wetting: when $\gamma_{\text {film }}<\gamma_{\text {sub }}$ it is energetically more favorable to cover the substrate with an infinitesimal layer of film atoms (and pay surface energy with density $\gamma_{\text {film }}$ ) rather than to leave any part of the substrate exposed (and pay surface energy with density $\gamma_{\text {sub }}$ ).

The asymptotic analysis, which relies on the notion of $\Gamma$-convergence introduced by De Giorgi, is very close in spirit to recent work of Bonnetier and Chambolle [4]. However it does not follow directly from their analysis. Here we have chosen to present a self-contained proof based on somewhat different arguments. We should mention that the results contained in [4] have been extended and generalized in a higher dimensional setting in the two recent papers [6] and [8].

In the second part of this work we restrict ourselves to the wetting regime $\gamma_{\text {film }}<\gamma_{\text {sub }}$ and to homogeneous anisotropic elastic materials, and we study the regularity of local minimizers $(\mathbf{u}, \Omega)$ of the limiting functional $F_{\infty}$ (see (3.1)), under a volume constraint.

Roughly speaking our main regularity results show that the profile $h$ of the film for a locally minimizing configuration is regular except for at most a finite number of cusps and "vertical cuts" which correspond to vertical cracks in the film. Numerical results obtained by Spencer and Meiron [27] confirm that steady state solutions exhibit cusp singularities, and also time-dependent evolution of small disturbances of the flat interface result in the formation of deep grooved cusps. Additional analytical evidence of the onset of cusps is provided in the work of Chiu and Gao [10] (see also [14]), where it was shown that the cycloid which minimizes the total energy (among a one-parameter family of cycloids) has a cusp singularity pointing toward the solid. Experimental validation of sharp cusplike features in $\mathrm{SI}_{0.6} \mathrm{Ge}_{0.4}$ and the discussion of possible mechanisms that may explain this phenomenon can be found in [9]. 
As a consequence we give a proof of the zero contact-angle condition between the wetting layer and islands, thus providing a rigorous mathematical justification to the formal argument used in [26] and based on matched asymptotic expansions. To the best of our knowledge these results are completely new in this context. The extension of some of these results to the three-dimensional case is the subject of a future work.

Let us now briefly describe the main steps in the regularity proof. As a starting point we observe that volume constrained minimizers of the limiting energy $F_{\infty}$ are also unconstrained local minimizers if we add to $F_{\infty}$ a suitable volume penalization. This allows us to consider arbitrary variations of $h$ and to prove, adapting an argument introduced in [7], a uniform interior sphere condition for the domain $\Omega$. This yields the conclusion that the graph of $h$ is a Lipschitz continuous curve away from a finite number of singular points. Having the Lipschitz continuity of $h$ in hand, a blow up argument, combined with classical results on corner domains for solutions of Lamé systems, leads to a precise decay estimate for the gradient of the displacement $\mathbf{u}$ near the boundary, which in turn implies the $C^{1, \alpha}$ regularity of $h$ and $\nabla \mathbf{u}$. At this point a bootstrap argument together with a theorem proved in [19] gives the final higher regularity result.

\section{Relaxation and $\Gamma$-convergence}

Throughout the paper we denote by $\mathbf{x}=(x, y)$ the generic point of $\mathbb{R}^{2}$ and by $B(\mathbf{x}, r)$ the open disc centered at $\mathbf{x}$ with radius $r$. Given two sets $A, B \subset \mathbb{R}^{2}$ their Hausdorff distance dist $\mathcal{H}(A, B)$ is defined as

$$
\operatorname{dist}_{\mathcal{H}}(A, B):=\inf \left\{r>0: A \subset N_{r}(B) \text { and } B \subset N_{r}(A)\right\} \text {, }
$$

where

$$
N_{r}(A):=\left\{\mathbf{x} \in \mathbb{R}^{2}:|\mathbf{x}-\mathbf{y}|<r \text { for some } \mathbf{y} \in A\right\}
$$

and $N_{r}(B)$ is defined similarly.

In the sequel $h$ is a lower semicontinuous function from $[a, b]$ with values in $[0,+\infty)$. Given $h$ let

$$
\begin{aligned}
& \Omega_{h}:=\{(x, y): a<x<b, y<h(x)\}, \\
& \Omega_{h}^{+}:=\{(x, y): a<x<b, 0<y<h(x)\}
\end{aligned}
$$

be the reference configuration of the substrate/film system and the reference configuration of the film, respectively. The set

$$
\Gamma_{h}:=\partial \Omega_{h} \cap((a, b) \times \mathbb{R}),
$$

represents the free profile of the film. We also consider the set

$$
\widetilde{\Gamma}_{h}:=\partial \bar{\Omega}_{h} \cap((a, b) \times \mathbb{R}) .
$$

When there is no risk of ambiguity we shall omit the subscript $h$ in the above notations.

We denote by $\operatorname{Var} h$ the pointwise variation of $h$, that is

$$
\begin{aligned}
\operatorname{Var} h:=\sup & \left\{\sum_{i=1}^{n}\left|h\left(x_{i}\right)-h\left(x_{i-1}\right)\right|:\right. \\
& \left.P:=\left\{x_{0}, \ldots, x_{n}\right\} \text { is a partition of }[a, b]\right\}<\infty .
\end{aligned}
$$

We recall that if $h$ has finite pointwise variation, then for every $x \in(a, b)$ we may define

$$
\begin{aligned}
& h^{-}(x):=\min \left\{h\left(x^{+}\right), h\left(x^{-}\right)\right\}=\liminf _{z \rightarrow x} h(z), \\
& h^{+}(x):=\max \left\{h\left(x^{+}\right), h\left(x^{-}\right)\right\}=\limsup _{z \rightarrow x} h(z),
\end{aligned}
$$


where

$$
h\left(x^{ \pm}\right):=\lim _{z \rightarrow x^{ \pm}} h(z) .
$$

It may be verified that the functions $h^{ \pm}$coincide with the approximate upper and lower limit of $h$ in the sense of Federer (see [2] for the definition).

In the following lemma we collect some well known facts for later use.

Lemma 2.1 Let $h:[a, b] \rightarrow[0,+\infty)$ be a lower semicontinuous function and let $\Gamma$ and $\widetilde{\Gamma}$ be the sets defined in (2.3) and (2.4), respectively. The function $h$ has finite pointwise variation if and only if $\mathcal{H}^{1}(\Gamma)<+\infty$. Moreover, if $h$ has finite pointwise variation then:

(i) the set $\Omega$ defined in (2.1) has finite perimeter in $(a, b) \times \mathbb{R}$,

(ii) $\Gamma=\left\{(x, y): a<x<b, h(x) \leq y \leq h^{+}(x)\right\}$,

(iii) the function $h^{-}$is lower semicontinuous and

$$
\stackrel{\circ}{\Omega}=\left\{(x, y): a<x<b, y<h^{-}(x)\right\},
$$

(iv) $\widetilde{\Gamma}=\left\{(x, y): a<x<b, h^{-}(x) \leq y \leq h^{+}(x)\right\}$.

Notice that (ii) and (iv) immediately imply that $\Gamma$ and $\widetilde{\Gamma}$ are connected.

We now introduce the space

$$
\begin{aligned}
X_{\text {Lip }}:=\left\{(\mathbf{u}, \Omega): \mathbf{u} \in H_{\mathrm{loc}}^{1}\left(\Omega ; \mathbb{R}^{2}\right), \Omega \text { is as in }(1.1),\right. \\
\quad h \text { is Lipschitz continuous }\},
\end{aligned}
$$

where the unrelaxed energies (1.6) and (1.10) are originally defined. In the next proposition we will show that energy bounded sequences in $X_{\text {Lip }}$ are compact in a larger space $X$ of admissible relaxed configurations defined as

$$
X:=\left\{(\mathbf{u}, \Omega): \mathbf{u} \in H_{\text {loc }}^{1}\left(\Omega ; \mathbb{R}^{2}\right), \Omega\right. \text { is in (1.1), }
$$

$h$ is lower semicontinuous and has finite pointwise variation $\}$.

We recall that an infinitesimal rigid motion is an affine displacement of the form $\mathbf{v}(\mathbf{x})=\mathbf{a}+\mathbf{B x}$, where $\mathbf{B}$ is a skew-symmetric matrix and $\mathbf{a}$ is a constant vector.

Proposition 2.2 (Compactness) Let $\left\{\left(\mathbf{u}_{n}, \Omega_{h_{n}}\right)\right\} \subset X_{\text {Lip }}$ be such that

$$
\sup _{n}\left(\int_{\Omega_{h_{n}}}\left|\mathbf{E}\left(\mathbf{u}_{n}\right)\right|^{2} d \mathbf{x}+\mathcal{H}^{1}\left(\Gamma_{h_{n}}\right)+\left|\Omega_{h_{n}}^{+}\right|\right)<\infty
$$

Then there exist a subsequence $\left\{\left(\mathbf{u}_{n_{k}}, \Omega_{h_{n_{k}}}\right)\right\}$, infinitesimal rigid motions $\mathbf{v}_{k}$, and $(\mathbf{u}, \Omega) \in X$ such that the sets $\mathbb{R}^{2} \backslash \Omega_{h_{n_{k}}}$ converge in the Hausdorff metric to $\mathbb{R}^{2} \backslash \Omega$ and the functions $u_{n_{k}}+\mathbf{v}_{k}$ converge to $u$ weakly in $H^{1}\left(\Omega^{\prime} ; \mathbb{R}^{2}\right)$ for every $\Omega^{\prime} \subset \subset \Omega$.

Proof. For simplicity we write $\Omega_{n}$ and $\Gamma_{n}$ in place of $\Omega_{h_{n}}$ and $\Gamma_{h_{n}}$, respectively. From the assumption it follows that

$$
\sup _{n}\left(\left|\Omega_{n}^{+}\right|+\mathcal{H}^{1}\left(\Gamma_{n}\right)\right)<\infty .
$$

Therefore for all $n$ we have $\Omega_{n} \subset\{(x, y): a<x<b, y<l\}$ for some $l>0$. Hence the compactness of the sets $\mathbb{R}^{2} \backslash \Omega_{n}$ is equivalent to the compactness of the equibounded sets $\{(x, y): a<x<$ $b, y \leq l\} \backslash \Omega_{n}$ which follows from Blaschke Compactness Theorem (see Theorem 6.1 in [2]). Thus 
we may assume that, up to a subsequence (not relabelled), $\mathbb{R}^{2} \backslash \Omega_{n}$ converges in the Hausdorff metric to a set $\mathbb{R}^{2} \backslash \Omega$. It is not difficult to see that $\Omega=\Omega_{h}$ where $h$ is the lower semicontinuous function given by

$$
h(x):=\inf \left\{\liminf _{n \rightarrow \infty} h_{n}\left(x_{n}\right): x_{n} \rightarrow x\right\} .
$$

By the same theorem we may also assume that $\left\{\bar{\Gamma}_{n}\right\}$ converges in the Hausdorff metric to some compact set $K$. It can be easily checked that $\Gamma \subset K$. Therefore by Gołąb's Theorem

$$
\mathcal{H}^{1}(\Gamma) \leq \mathcal{H}^{1}(K) \leq \liminf _{n \rightarrow \infty} \mathcal{H}^{1}\left(\Gamma_{n}\right)
$$

Hence from Lemma 2.1 the function $h$ has finite pointwise variation.

Moreover, by (2.9) we may find a subsequence (not relabelled) and a function $\mathbf{E}_{\infty} \in L^{2}\left(\Omega ; \mathbb{R}_{\text {sym }}^{2 \times 2}\right)$ such that

$$
\mathbf{E}\left(\mathbf{u}_{n}\right) \chi_{\Omega_{n}} \rightarrow \mathbf{E}_{\infty} \chi_{\Omega} \text { in } L^{2}\left(\mathbb{R}^{2} ; \mathbb{R}_{\mathrm{sym}}^{2 \times 2}\right) .
$$

Fix a ball $B \subset\{(x, y): a<x<b, y<0\}$. By adding suitable infinitesimal rigid motions, if needed, without loss of generality we may assume that

$$
\int_{B} \mathbf{u}_{n} d \mathbf{x}=\mathbf{0}, \quad \int_{B}\left(\nabla \mathbf{u}_{n}-\nabla \mathbf{u}_{n}^{T}\right) d \mathbf{x}=\mathbf{0}
$$

for every $n$, in addition to (2.9). Note that here we have used the fact that $B \subset \Omega_{n}$.

Construct a sequence of bounded open sets $\left\{D_{j}\right\}$ with Lipschitz boundary such that

$$
B \subset D_{j} \subset \subset \Omega, \quad D_{j} \nearrow \Omega
$$

as $j \rightarrow \infty$. By (2.11) and Korn's inequality combined with a standard diagonalization argument, there exists $\mathbf{u} \in H_{\text {loc }}^{1}\left(\Omega ; \mathbb{R}^{2}\right)$ with $\mathbf{E}(\mathbf{u})=\mathbf{E}_{\infty}$ such that $\mathbf{u}_{n} \rightarrow \mathbf{u}$ weakly in $H^{1}\left(D_{j} ; \mathbb{R}^{2}\right)$ for every $j$. As the pair $\left(\mathbf{u}, \Omega_{h}\right) \in X$ this concludes the proof.

The previous proposition motivates the following notion of convergence.

Definition 2.3 A sequence $\left\{\left(u_{n}, \Omega_{h_{n}}\right)\right\} \subset X$ is said to converge to $(u, \Omega)$ in $X$ if:

(i) the functions $h_{n}$ have equibounded variations; i.e., $\sup _{n} \operatorname{Var} h_{n}<+\infty$;

(ii) the sets $\mathbb{R}^{2} \backslash \Omega_{h_{n}}$ converge in the Hausdorff metric to $\mathbb{R}^{2} \backslash \Omega$;

(iii) the functions $u_{n}$ converge to $u$ weakly in $H^{1}\left(\Omega^{\prime} ; \mathbb{R}^{2}\right)$ for every $\Omega^{\prime} \subset \subset \Omega$. hold.

We will often write $\left(u_{n}, \Omega_{h_{n}}\right) \rightarrow(u, \Omega)$ to mean that (i), (ii), and (iii) of the previous definition

Note that from the Hausdorff convergence of $\left\{\mathbb{R}^{2} \backslash \Omega_{h_{n}}\right\}$ to $\mathbb{R}^{2} \backslash \Omega$ it follows that $\Omega^{\prime} \subset \Omega_{h_{n}}$ for all $n$ sufficiently large. Hence condition (iii) in the previous definition makes sense.

Remark 2.4 We observe that condition (i) in Definition 2.3 is equivalent to requiring that $\sup _{n} \mathcal{H}^{1}\left(\Gamma_{h_{n}}\right)<+\infty$.

The following lemma shows a useful property of the convergence in $X$.

Lemma 2.5 Assume that (i) and (ii) of Definition 2.3 hold. Then $h_{n}$ converges to $h$ in $L^{1}(a, b)$ and, in particular,

$$
\left|\Omega_{h_{n}} \Delta \Omega\right| \rightarrow 0 .
$$


Proof. For simplicity we write $\Omega_{n}$ and $\Gamma_{n}$ in place of $\Omega_{h_{n}}$ and $\Gamma_{h_{n}}$, respectively. Since the functions $h_{n}$ have equibounded variations, up to extracting a subsequence there exists $\tilde{h}$ with bounded variation in $(a, b)$ such that $h_{n} \rightarrow \tilde{h}$ in $L^{1}(a, b)$ and everywhere in $(a, b) \backslash N_{0}$, with $N_{0}$ at most countable by the Helly theorem (see [22]). Hence the lemma amounts to showing that $\tilde{h}=h$ almost everywhere in $(a, b)$.

By the Blaschke Compactness Theorem (see Theorem 6.1 in [2]) we may also assume that

$$
\bar{\Gamma}_{n} \rightarrow K \quad \text { in the Hausdorff distance }
$$

for some compact connected set $K$. Moreover, by the Gołąb Theorem and by Remark 2.4 it follows that

$$
\mathcal{H}^{1}(K) \leq \liminf _{n \rightarrow \infty} \mathcal{H}^{1}\left(\Gamma_{n}\right)<+\infty
$$

Denote by $K_{x}:=\{(x, y) \in K\}$ the vertical section of $K$ corresponding to the point $x \in(a, b)$. We claim that $\mathcal{H}^{1}\left(K_{x}\right)=0$ for all $x \in(a, b) \backslash N_{1}$, with $N_{1}$ at most countable. To see this it is enough to observe that

$$
\frac{1}{n} \operatorname{card}\left\{x: \mathcal{H}^{1}\left(K_{x}\right) \geq \frac{1}{n}\right\} \leq \sum_{x: \mathcal{H}^{1}\left(K_{x}\right) \geq \frac{1}{n}} \mathcal{H}^{1}\left(K_{x}\right) \leq \mathcal{H}^{1}(K)<\infty
$$

Since $K$ is the Hausdorff limit of graphs, each $K_{x}$ is connected and so $K_{x}$ reduces to one point for all $x \in(a, b) \backslash N_{1}$. Set $N:=N_{0} \cup N_{1}$. In order to conclude the proof it suffices to show that for all $x \in(a, b) \backslash N$ the equality $\tilde{h}(x)=h(x)$ holds. Assume by contradiction that $\tilde{h}(x) \neq h(x)$ for some $x \in(a, b) \backslash N$. Since $\tilde{h}(x)=\lim _{n} h_{n}(x)$, by (2.10) we deduce that $\tilde{h}(x)>h(x)$ and that there exists a (sub)sequence $x_{n} \rightarrow x$ with $h_{n}\left(x_{n}\right) \rightarrow h(x)$. Note that for any $y \in(h(x), \tilde{h}(x))$ we have $h_{n}\left(x_{n}\right)<y<h_{n}(x)$ for $n$ large enough. Hence, using the connectedness of $\Gamma_{n}$, we may find $x_{n}^{\prime}$ between $x$ and $x_{n}$ such that $\left(x_{n}^{\prime}, y\right) \in \Gamma_{n}$. Since clearly $x_{n}^{\prime} \rightarrow x$, we deduce from (2.12) that $(x, y) \in K$, that is, $y \in K_{x}$. Therefore $(h(x), \tilde{h}(x)) \subset K_{x}$, but this is a contradiction since by our choice of $x$ the section $K_{x}$ reduces to one point.

Remark 2.6 Combining Lemma 2.1, Remark 2.4, and Lemma 2.5 we deduce that if $\left(u_{n}, \Omega_{h_{n}}\right) \rightarrow$ $(u, \Omega)$ in $X$, then the sets $\Omega_{h_{n}}$ have equibounded perimeters in $(a, b) \times \mathbb{R}, \chi_{\Omega_{h_{n}}} \rightarrow \chi_{\Omega}$ in $L_{\mathrm{loc}}^{1}((a, b) \times$ $\mathbb{R})$, and $D \chi_{\Omega_{h_{n}}} \stackrel{*}{\rightarrow} D \chi_{\Omega}$ weakly* in the sense of measures.

If $h$ has finite pointwise variation then the upper boundary $\Gamma$ of $\Omega$ defined in (1.7) may be represented as the union of three subsets

$$
\Gamma=\Gamma_{\text {vert }} \cup \Gamma_{\text {cuts }} \cup \Gamma_{\text {graph }},
$$

where:

1. $\Gamma_{\text {vert }}$ is the closure of the (at most) countable collection of vertical segments corresponding to the jumps of $h$, that is

$$
\Gamma_{\text {vert }}:=\overline{\left\{(x, y): x \in(a, b) \cap S(h), h^{-}(x) \leq y \leq h^{+}(x)\right\}},
$$

where, as usual,

$$
S(h):=\left\{x \in(a, b): h^{-}(x) \neq h^{+}(x)\right\}
$$

2. $\Gamma_{\text {cuts }}$ is given by the union of a (at most) countable number of vertical cuts which correspond to the points where $h \neq h^{-}$, precisely

$$
\Gamma_{\text {cuts }}:=\left\{(x, y): x \in(a, b) \cap S, h(x) \leq y \leq h^{-}(x)\right\},
$$

with

$$
S:=\left\{x \in(a, b): h(x)<h^{-}(x)\right\}
$$


3. $\Gamma_{\text {graph }}:=\Gamma \backslash\left(\Gamma_{\text {vert }} \cup \Gamma_{\text {cuts }}\right)$ is the portion of the $\Gamma$ corresponding to regular part of the graph of $h$.

Thus $\widetilde{\Gamma}$ (see (2.4) ) can obtained from $\Gamma$ by eliminating the vertical cuts (i.e. by re-defining $h$ to be $h^{-}$), and we have

$$
\Gamma=\widetilde{\Gamma} \cup \Gamma_{\text {cuts }}
$$

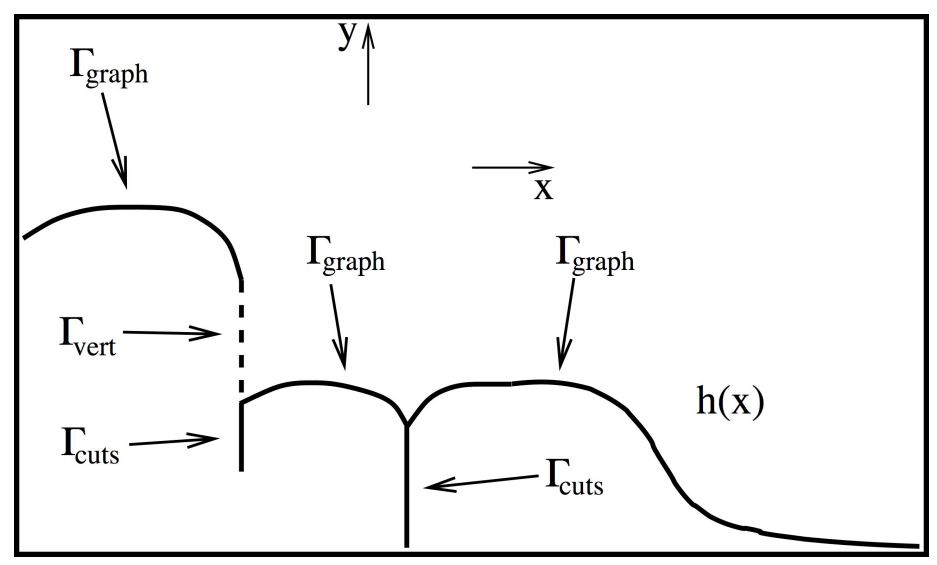

Figure 1: An admissible relaxed configuration.

The compactness and the $\Gamma$-convergence results proved below are very similar to those established by Bonnetier and Chambolle in [4]. However our proofs do not follow directly from the analysis in [4] and make use of different arguments except for the next lemma.

Lemma 2.7 Let $h:[a, b] \rightarrow[0,+\infty)$ be a lower semicontinuous function with finite pointwise variation, such that $h^{-}=h$. Define the Yosida transform

$$
h_{n}(x):=\inf \left\{h\left(x^{\prime}\right)+n\left|x-x^{\prime}\right|: x^{\prime} \in[a, b]\right\}
$$

for $x \in[a, b]$. Then $\mathbb{R}^{2} \backslash \Omega_{h_{n}} \rightarrow \mathbb{R}^{2} \backslash \Omega$ in the Hausdorff metric and

$$
\lim _{n \rightarrow \infty} \mathcal{H}^{1}\left(\Gamma_{h_{n}}\right)=\mathcal{H}^{1}(\Gamma)
$$
$[4])$.

The proof of this lemma is contained in the proof of Lemma 2.1 of [4] (given in Section 5.1 of

Next we give a representation formula of the relaxed functional $\bar{F}_{\delta}(\mathbf{u}, \Omega)$ of $F_{\delta}(\mathbf{u}, \Omega)$.

Theorem 2.8 (Relaxation) For every $\delta>0$ let $\bar{F}_{\delta}$ be the relaxed functional of $F_{\delta}$ under volume constraint, i.e.,

$$
\begin{array}{r}
\bar{F}_{\delta}(\mathbf{u}, \Omega)=\inf \left\{\liminf _{n \rightarrow \infty} F_{\delta}\left(\mathbf{u}_{n}, \Omega_{h_{n}}\right):\left(\mathbf{u}_{n}, \Omega_{h_{n}}\right) \in X_{\mathrm{Lip}},\right. \\
\left.\left(\mathbf{u}_{n}, \Omega_{h_{n}}\right) \rightarrow(\mathbf{u}, \Omega) \text { in } X,\left|\Omega_{h_{n}}^{+}\right|=\left|\Omega^{+}\right|\right\}
\end{array}
$$

for all $(\mathbf{u}, \Omega) \in X$. Then

$$
\bar{F}_{\delta}(\mathbf{u}, \Omega)=\int_{\Omega} W\left(\mathbf{E}(\mathbf{u})(\mathbf{x})-\mathbf{E}_{\delta}(y)\right) d \mathbf{x}+\int_{\widetilde{\Gamma}} \varphi_{\delta}(y) d \mathcal{H}^{1}(\mathbf{x})+2 \sum_{x \in S} \int_{h(x)}^{h^{-}(x)} \varphi_{\delta}(y) d y
$$

where $\widetilde{\Gamma}$ and $S$ are the set defined in (2.4) and (2.17), respectively. 
Proof. For simplicity we write $\Omega_{n}$ and $\Gamma_{n}$ in place of $\Omega_{h_{n}}$ and $\Gamma_{h_{n}}$, respectively. Fix $(\mathbf{u}, \Omega) \in X$. Step 1: Let $\mathcal{F}_{\delta}(\mathbf{u}, \Omega)$ denote the right-hand side of (2.18). We begin by showing that

$$
\bar{F}_{\delta}(\mathbf{u}, \Omega) \geq \mathcal{F}_{\delta}(\mathbf{u}, \Omega) .
$$

Consider a sequence $\left\{\left(\mathbf{u}_{n}, \Omega_{n}\right)\right\} \subset X_{\text {Lip }}$ such that $\left(\mathbf{u}_{n}, \Omega_{n}\right) \rightarrow(\mathbf{u}, \Omega)$ in $X$, with $\left|\Omega_{n}^{+}\right|=\left|\Omega^{+}\right|$, and let $h_{n}$ and $h$ be the functions associated with $\Omega_{n}$ and $\Omega$, respectively. Without loss of generality we may assume that

$$
\liminf _{n \rightarrow \infty} F_{\delta}\left(\mathbf{u}_{n}, \Omega_{n}\right)=\lim _{n \rightarrow \infty} F_{\delta}\left(\mathbf{u}_{n}, \Omega_{n}\right)<\infty
$$

and

$$
\bar{\Gamma}_{n} \rightarrow K \quad \text { in the Hausdorff distance, }
$$

for some compact set $K$ containing $\Gamma$.

Since $h$ is of pointwise bounded variation and $\mathcal{H}^{1}(\Gamma)<\infty$, the set $S$ defined in (2.17) is at most countable. Fix $k \in \mathbb{N}$ (with $k$ the cardinality of $S$ if $S$ is finite) and let $S^{(k)}:=\left\{x_{1}, \cdots, x_{k}\right\}$ be any subset of $k$ elements of $S$. Without loss of generality we may assume that

$$
a<x_{1}<\cdots<x_{k}<b .
$$

Since the Hausdorff convergence of compact sets is equivalent to the Kuratowski convergence (see [2]) for each fixed $j=1, \cdots, k$ we may find a sequence $\left\{x_{n, j}\right\} \subset(a, b)$ such that

$$
x_{n, j} \rightarrow x_{j}, \quad h_{n}\left(x_{n, j}\right) \rightarrow h\left(x_{j}\right)
$$

as $n \rightarrow \infty$. The following construction is borrowed from [21]. Let $x_{n}, x_{0}:(a, b+1) \rightarrow(a, b)$ be the (continuous) piecewise affine functions such that $x_{n}(a)=x_{0}(a)=a$,

$$
\begin{aligned}
& x_{n}^{\prime}(s):= \begin{cases}0 & s \in\left(x_{n, j}+\frac{j-1}{k}, x_{n, j}+\frac{j}{k}\right) \quad j=1, \cdots, k, \\
1 & \text { otherwise, }\end{cases} \\
& x_{0}^{\prime}(s):= \begin{cases}0 & s \in\left(x_{j}+\frac{j-1}{k}, x_{j}+\frac{j}{k}\right) \quad j=1, \cdots, k, \\
1 & \text { otherwise, }\end{cases}
\end{aligned}
$$

and define $\hat{h}_{n}:=h_{n} \circ x_{n}$ and

$$
\hat{h}(s):= \begin{cases}h\left(x_{j}\right) & s \in\left(x_{j}+\frac{j-1}{k}, x_{j}+\frac{j}{k}\right) \quad j=1, \cdots, k, \\ h^{-}\left(x_{0}(s)\right) & \text { otherwise. }\end{cases}
$$

Note that $\hat{h}_{n}(s)=h_{n}\left(x_{n, j}\right)$ for $s \in\left(x_{n, j}+\frac{j-1}{k}, x_{n, j}+\frac{j}{k}\right), j=1, \cdots, k$. Denote by $\widehat{\mathcal{R}}$ the open strip $(a, b+1) \times \mathbb{R}$ and set

$$
\widehat{\Omega}_{n}:=\left\{(s, y): a<s<b+1, y<\hat{h}_{n}(s)\right\}, \quad \widehat{\Omega}:=\{(s, y): a<s<b+1, y<\hat{h}(s)\}
$$

and

$$
\widehat{\Gamma}_{n}:=\partial \widehat{\Omega}_{n} \cap \widehat{\mathcal{R}}, \quad \widehat{\Gamma}:=\partial \widehat{\Omega} \cap \widehat{\mathcal{R}} .
$$

Then by construction and since $\varphi_{\delta}$ is independent of the $x$ variable it can be shown that

$$
\begin{aligned}
\int_{\widehat{\Gamma}_{n}} \varphi_{\delta}(y) d \mathcal{H}^{1}(\mathbf{x}) & =\int_{\Gamma_{n}} \varphi_{\delta}(y) d \mathcal{H}^{1}(\mathbf{x})+\frac{1}{k} \sum_{j=1}^{k} \varphi_{\delta}\left(h_{n}\left(x_{n, j}\right)\right) \\
\int_{\widehat{\Gamma}} \varphi_{\delta}(y) d \mathcal{H}^{1}(\mathbf{x}) & =\int_{\widetilde{\Gamma}} \varphi_{\delta}(y) d \mathcal{H}^{1}(\mathbf{x})+2 \sum_{j=1}^{k} \int_{h\left(x_{j}\right)}^{h^{-}\left(x_{j}\right)} \varphi_{\delta}(y) d y \\
& +\frac{1}{k} \sum_{j=1}^{k} \varphi_{\delta}\left(h\left(x_{j}\right)\right) .
\end{aligned}
$$


By (2.21) we have that

$$
\lim _{n \rightarrow \infty} \sum_{j=1}^{k} \varphi_{\delta}\left(h_{n}\left(x_{n, j}\right)\right)=\sum_{j=1}^{k} \varphi_{\delta}\left(h\left(x_{j}\right)\right) .
$$

It is not difficult to see that $\widehat{\Omega}_{n}$ and $\widehat{\Omega}$ are sets of finite perimeter in the open strip $\widehat{\mathcal{R}}$, that $\chi_{\widehat{\Omega}_{n}} \rightarrow \chi_{\widehat{\Omega}}$ in $L_{\text {loc }}^{1}\left(\mathbb{R}^{2}\right)$, and that

$$
D \chi_{\widehat{\Omega}_{n}} \stackrel{*}{\rightarrow} D \chi_{\widehat{\Omega}}
$$

weakly* in the sense of measures. Applying Reshetnyak's Lower Semicontinuity Theorem (as stated in Theorem 2.38 in [2] with $\mu_{n}:=D \chi_{\widehat{\Omega}_{n}}, \mu:=D \chi_{\widehat{\Omega}}$ and $\left.f(\mathbf{x}, \xi)=\varphi_{\delta}(y)|\xi|\right)$ we obtain

$$
\liminf _{n \rightarrow \infty} \int_{\widehat{\Gamma}_{n}} \varphi_{\delta}(y) d \mathcal{H}^{1}(\mathbf{x}) \geq \int_{\mathcal{F} \widehat{\Omega}} \varphi_{\delta}(y) d \mathcal{H}^{1}(\mathbf{x})
$$

where $\mathcal{F} \widehat{\Omega}$ denotes the reduced boundary of $\widehat{\Omega}$ in $\widehat{R}$ (see Def. 3.54 in [2]). Note that we have used the fact that

$$
\left|D \chi_{\widehat{\Omega}_{n}}\right|(\widehat{R})=\mathcal{H}^{1}\left\lfloor\widehat{\Gamma}_{n}, \quad\left|D \chi_{\widehat{\Omega}}\right|(\widehat{R})=\mathcal{H}^{1}\lfloor\mathcal{F} \widehat{\Omega},\right.
$$

as proved in Proposition 3.62 and Theorem 3.59 in [2]. It is well known that (see [13, Theorem 4.5.9 (5)]), up to a set of $\mathcal{H}^{1}$ measure zero, we have

$$
\mathcal{F} \widehat{\Omega}=\left\{(s, y): a<s<b+1, \hat{h}^{-}(s) \leq y \leq \hat{h}^{+}(s)\right\}=\widehat{\Gamma},
$$

where the functions $\hat{h}^{ \pm}$are defined as in (2.7)-(2.6) with $\hat{h}$ in place of $h$. Hence the inequality (2.24) is equivalent to

$$
\liminf _{n \rightarrow \infty} \int_{\widehat{\Gamma}_{n}} \varphi_{\delta}(y) d \mathcal{H}^{1}(\mathbf{x}) \geq \int_{\widehat{\Gamma}} \varphi_{\delta}(y) d \mathcal{H}^{1}(\mathbf{x}) .
$$

By (2.22) and (2.23) we have that

$$
\liminf _{n \rightarrow \infty} \int_{\Gamma_{n}} \varphi_{\delta}(y) d \mathcal{H}^{1}(\mathbf{x}) \geq \int_{\widetilde{\Gamma}} \varphi_{\delta}(y) d \mathcal{H}^{1}(\mathbf{x})+2 \sum_{x \in S^{(k)}} \int_{h(x)}^{h^{-}(x)} \varphi_{\delta}(y) d y .
$$

If $S$ is infinite we now let $k \rightarrow \infty$ in the previous inequality to conclude that

$$
\liminf _{n \rightarrow \infty} \int_{\Gamma_{n}} \varphi_{\delta}(y) d \mathcal{H}^{1}(\mathbf{x}) \geq \int_{\widetilde{\Gamma}} \varphi_{\delta}(y) d \mathcal{H}^{1}(\mathbf{x})+2 \sum_{x \in S} \int_{h(x)}^{h^{-}(x)} \varphi_{\delta}(y) d y .
$$

Note that in view (2.20), by extracting a subsequence, if necessary, we may assume that the limit inferior in (2.26) is actually a limit.

It remains to study the bulk energy. Fix any $D \subset \subset \Omega$. Since the sequence $\mathbf{u}_{n}$ converges weakly to $\mathbf{u}$ in $H^{1}\left(D ; \mathbb{R}^{2}\right)$ we have that

$$
\mathbf{E}\left(\mathbf{u}_{n}\right) \rightarrow \mathbf{E}(\mathbf{u}) \text { in } L^{2}\left(D ; \mathbb{R}^{2 \times 2}\right) .
$$

Hence

$$
\begin{aligned}
\liminf _{n \rightarrow \infty} \int_{\Omega_{n}} W\left(\mathbf{E}\left(\mathbf{u}_{n}\right)-\mathbf{E}_{\delta}\right) d \mathbf{x} & \geq \liminf _{n \rightarrow \infty} \int_{D} W\left(\mathbf{E}\left(\mathbf{u}_{n}\right)-\mathbf{E}_{\delta}\right) d \mathbf{x} \\
& \geq \int_{D} W\left(\mathbf{E}(\mathbf{u})-\mathbf{E}_{\delta}\right) d \mathbf{x} .
\end{aligned}
$$


By letting $D \nearrow \Omega$ and recalling (2.20) and (2.26) (with the limit inferior replaced by a limit) we conclude that (2.19) holds.

Step 2: To prove the reverse inequality

$$
\mathcal{F}_{\delta}(\mathbf{u}, \Omega) \geq \bar{F}_{\delta}(\mathbf{u}, \Omega),
$$

it is enough to construct a sequence $\left\{h_{n}\right\}$ of Lipschitz continuous functions such that $0 \leq h_{n} \leq h$,

$$
\left(\mathbf{u}, \Omega_{n}\right) \rightarrow(\mathbf{u}, \Omega) \quad \text { and } \quad \lim _{n \rightarrow \infty} F_{\delta}\left(\mathbf{u}, \Omega_{n}\right)=\mathcal{F}_{\delta}(\mathbf{u}, \Omega),
$$

where $\Omega_{n}:=\Omega_{h_{n}}$.

Indeed, assume that (2.29) holds with $h_{n} \leq h$. Then by a standard slicing argument we fix $y_{0}<0$ such that $\mathbf{u}\left(\cdot, y_{0}\right) \in H^{1}\left((a, b) ; \mathbb{R}^{2}\right)$ and define

$$
\mathbf{u}_{n}(\mathbf{x}):= \begin{cases}\mathbf{u}\left(x, y-\varepsilon_{n}\right) & \text { if } y>y_{0}+\varepsilon_{n} \\ \mathbf{u}\left(x, y_{0}\right) & \text { if } y_{0}<y \leq y_{0}+\varepsilon_{n} \\ \mathbf{u}(x, y) & \text { if } y \leq y_{0}\end{cases}
$$

and

$$
\tilde{h}_{n}(x):=h_{n}(x)+\varepsilon_{n},
$$

where

$$
\varepsilon_{n}:=\frac{1}{b-a}\left(\left|\Omega^{+}\right|-\int_{a}^{b} h_{n}(x) d x\right) .
$$

Note that since $\left|\Omega_{n}^{+}\right| \rightarrow\left|\Omega^{+}\right|$we have that $\varepsilon_{n} \rightarrow 0$. Clearly $\left|\Omega_{\tilde{h}_{n}}^{+}\right|=\left|\Omega^{+}\right|,\left(\mathbf{u}_{n}, \Omega_{\tilde{h}_{n}}\right) \rightarrow(\mathbf{u}, \Omega)$ in $X$ and

$$
\lim _{n \rightarrow \infty} F_{\delta}\left(\mathbf{u}, \Omega_{n}\right)=\lim _{n \rightarrow \infty} F_{\delta}\left(\mathbf{u}_{n}, \Omega_{\tilde{h}_{n}}\right) .
$$

Hence (2.28) will follow from (2.29). The remaining of the proof is devoted to the construction of the sequence $\left\{h_{n}\right\}$.

Step 3: Assume first that

$$
h^{-}=h .
$$

We denote by $h_{n}$ the Yosida transform of $h$ defined as in Lemma 2.7. It is easy to see that $0 \leq h_{n} \leq h_{n+1} \leq h$ and that $h_{n}$ is Lipschitz. Let $\Omega_{n}$ be the sets associated with $h_{n}$. We claim that

$$
\lim _{n \rightarrow \infty} F_{\delta}\left(\mathbf{u}, \Omega_{n}\right)=\mathcal{F}_{\delta}(\mathbf{u}, \Omega) .
$$

The convergence of the bulk energies of the approximating sequence follows immediately from Lebesgue Monotone Convergence Theorem. Thus it remains to prove the convergence of the surface energies.

From Lemma 2.7 and by (2.30) we have that

$$
\lim _{n \rightarrow \infty} \mathcal{H}^{1}\left(\Gamma_{n}\right)=\mathcal{H}^{1}(\Gamma)=\mathcal{H}^{1}(\widetilde{\Gamma}) .
$$

Clearly $\Omega_{n}$ and $\Omega$ are sets of finite perimeter in the strip $\mathcal{R}=(a, b) \times \mathbb{R}$ and $\chi_{\Omega_{n}} \rightarrow \chi_{\Omega}$ in $L_{\text {loc }}^{1}\left(\mathbb{R}^{2}\right)$. As in (2.25), up to a set of $\mathcal{H}^{1}$ measure zero, we have

$$
\mathcal{F} \Omega=\left\{(x, y): a<x<b, h^{-}(x) \leq y \leq h^{+}(x)\right\}=\Gamma .
$$

Therefore, by (2.33) and (2.32) we obtain

$$
\left|D \chi_{\Omega_{n}}\right|(\mathcal{R})=\mathcal{H}^{1}\left(\Gamma_{n}\right) \rightarrow\left|D \chi_{\Omega}\right|(\mathcal{R})=\mathcal{H}^{1}(\Gamma) .
$$


Thus, arguing as in (2.24), we may apply Reshetnyak's Continuity Theorem (see Theorem 2.39 in [2]) to conclude that

$$
\lim _{n \rightarrow \infty} \int_{\Gamma_{n}} \varphi_{\delta}(y) d \mathcal{H}^{1}(\mathbf{x})=\int_{\Gamma} \varphi_{\delta}(y) d \mathcal{H}^{1}(\mathbf{x})
$$

Step 4: We consider next the case where the set $S$ defined in $(2.17)$ is finite, say $S=\left\{x_{1}, \cdots, x_{k}\right\}$, where as before

$$
a=: x_{0}<x_{1}<\cdots<x_{k}<b=: x_{k+1} .
$$

We claim that there exists an increasing sequence of lower semicontinuous functions $h_{n}$ satisfying (2.30) such that $\mathbb{R}^{2} \backslash \Omega_{n} \rightarrow \mathbb{R}^{2} \backslash \Omega$ in the Hausdorff metric and

$$
\lim _{n \rightarrow \infty} \mathcal{F}_{\delta}\left(\mathbf{u}, \Omega_{n}\right)=\mathcal{F}_{\delta}(\mathbf{u}, \Omega)
$$

To see this, let

$$
\epsilon_{0}:=\min \left\{\left|x_{j}-x_{j-1}\right|: j=1, \cdots, k+1\right\}>0
$$

and for $n \in \mathbb{N}$ define

$$
h_{n}(x):= \begin{cases}h\left(x_{j}\right) & x \in\left[x_{j}-\frac{\epsilon_{0}}{2 n}, x_{j}+\frac{\epsilon_{0}}{2 n}\right] \quad j=1, \cdots, k \\ h(x) & \text { otherwise. }\end{cases}
$$

Since $h$ is lower semicontinuous, and by the definition of $S$ (see (2.17)), for all $n$ sufficiently large we have that $h_{n}$ is lower semicontinuous and

$$
0 \leq h_{n} \leq h_{n+1} \leq h
$$

$h_{n} \rightarrow h$ pointwise and clearly $h_{n}^{-}=h_{n}$. It is easy to see that

$$
\begin{aligned}
\int_{\Gamma_{n}} \varphi_{\delta}(y) d \mathcal{H}^{1}(\mathbf{x}) & =\int_{\Gamma_{n} \cap \Gamma} \varphi_{\delta}(y) d \mathcal{H}^{1}(\mathbf{x}) \\
& +\sum_{j=1}^{k}\left(\int_{h\left(x_{j}\right)}^{h\left(x_{j}+\frac{\epsilon_{0}}{2 n}\right)} \varphi_{\delta}(y) d y+\int_{h\left(x_{j}\right)}^{h\left(x_{j}-\frac{\epsilon_{0}}{2 n}\right)} \varphi_{\delta}(y) d y\right) \\
& +\frac{\epsilon_{0}}{n} \sum_{j=1}^{k} \varphi_{\delta}\left(h\left(x_{j}\right)\right) .
\end{aligned}
$$

Since $h\left(x_{j}+\frac{\epsilon_{0}}{2 n}\right) \rightarrow h\left(x_{j}^{+}\right), h\left(x_{j}-\frac{\epsilon_{0}}{2 n}\right) \rightarrow h\left(x_{j}^{-}\right)$for each $j=1, \cdots, k$, we obtain that

$$
\int_{h\left(x_{j}\right)}^{h\left(x_{j}+\frac{\epsilon_{0}}{2 n}\right)} \varphi_{\delta}(y) d y+\int_{h\left(x_{j}\right)}^{h\left(x_{j}-\frac{\epsilon_{0}}{2 n}\right)} \varphi_{\delta}(y) d y \rightarrow 2 \int_{h\left(x_{j}\right)}^{h^{-}\left(x_{j}\right)} \varphi_{\delta}(y) d y+\int_{h^{-}\left(x_{j}\right)}^{h^{+}\left(x_{j}\right)} \varphi_{\delta}(y) d y
$$

and thus also by the Lebesgue Monotone Convergence Theorem we obtain (2.34).

Step 5: Finally, if the set $S$ defined in (2.17) is denumerable then there exists an increasing sequence of lower semicontinuous functions $h_{n}$ satisfying the hypotheses of previous step such that $\mathbb{R}^{2} \backslash \Omega_{n} \rightarrow \mathbb{R}^{2} \backslash \Omega$ in the Hausdorff metric and

$$
\lim _{n \rightarrow \infty} \mathcal{F}_{\delta}\left(\mathbf{u}, \Omega_{n}\right)=\mathcal{F}_{\delta}(\mathbf{u}, \Omega) .
$$

Indeed, for $x \in(a, b)$ and $n \in \mathbb{N}$ define

$$
h_{n}(x):=\min \left\{\max \left\{h^{-}(x)-\frac{1}{n}, 0\right\}, h(x)\right\} .
$$

Since $h$ is a function of finite pointwise variation, the set

$$
T_{n}:=\left\{x \in(a, b): h^{-}(x)-h(x) \geq \frac{1}{n}\right\}
$$


is finite. Note that $h_{n}$ is lower semicontinuous. Moreover, as $\left\{x \in(a, b): h_{n}^{-}(x) \neq h_{n}(x)\right\} \subset T_{n}$, the function $h_{n}$ satisfies the conditions of previous step. Clearly $h_{n} \nearrow h$ and

$$
\begin{aligned}
& \int_{\widetilde{\Gamma}_{n}} \varphi_{\delta}(y) d \mathcal{H}^{1}(\mathbf{x})+2 \sum_{h_{n}(x) \neq h_{n}^{-}(x)} \int_{h_{n}(x)}^{h_{n}^{-}(x)} \varphi_{\delta}(y) d y \\
& =\int_{\widetilde{\Gamma}} \varphi_{\delta}\left(\max \left\{y-\frac{1}{n}, 0\right\}\right) d \mathcal{H}^{1}(\mathbf{x})+2 \sum_{x \in T_{n}} \int_{h(x)}^{h^{-}(x)-\frac{1}{n}} \varphi_{\delta}(y) d y
\end{aligned}
$$

and thus by the Lebesgue Dominated Convergence Theorem we easily get (2.35) .

Combining Step 3, Step 4, and Step 5, and using a standard diagonalization argument, we obtain (2.28) . This concludes the proof of the theorem.

We are now ready to study the $\Gamma$-convergence in $X$, under a volume constraint, of the family $\left\{F_{\delta}\right\}_{\delta>0}$ as $\delta \rightarrow 0^{+}$(for the definition and the properties of $\Gamma$-convergence see [5] and [11]). Observe that the pointwise limit of $\varphi_{\delta}$ is the function $\varphi_{0}$ defined in (1.5), which is not lower semicontinuous in the wetting regime $\gamma_{\text {film }}<\gamma_{\text {sub }}$. Hence in the $\Gamma$-limit we expect the surface energy density to be given by its lower semicontinuous envelope

$$
\tilde{\varphi}(y):= \begin{cases}\gamma_{\text {film }} & \text { if } y>0 \\ \min \left\{\gamma_{\text {film }}, \gamma_{\text {sub }}\right\} & \text { if } y=0\end{cases}
$$

Indeed we have the following:

Theorem 2.9 ( $\Gamma$-convergence) For all $(\mathbf{u}, \Omega) \in X$ set

$$
\begin{aligned}
F_{\infty}(\mathbf{u}, \Omega):= & \int_{\Omega} W\left(\mathbf{E}(\mathbf{u})(\mathbf{x})-\mathbf{E}_{0}(y)\right) d \mathbf{x}+\min \left\{\gamma_{\text {film }}, \gamma_{\text {sub }}\right\} \mathcal{H}^{1}(\widetilde{\Gamma} \cap\{y=0\}) \\
& +\gamma_{\text {film }} \mathcal{H}^{1}(\widetilde{\Gamma} \backslash\{y=0\})+2 \gamma_{\text {film }} \sum_{x \in S}\left(h^{-}(x)-h(x)\right)
\end{aligned}
$$

where $\mathbf{E}_{0}$ is defined in (1.2) and the sets $\widetilde{\Gamma}$ and $S$ are defined in (2.4) and (2.17), respectively. Then $F_{\infty}$ is the $\Gamma$-limit in $X$ as $\delta \rightarrow 0^{+}$of the family $\left\{F_{\delta}\right\}_{\delta>0}$, under volume constraint.

Proof. We divide the proof into two steps.

Step 1: We start by showing that for all sequences $\delta_{n} \searrow 0,\left(\mathbf{u}_{n}, \Omega_{n}\right) \rightarrow(\mathbf{u}, \Omega)$ in $X$ with $\left(\mathbf{u}_{n}, \Omega_{n}\right) \in X_{\text {Lip }}$ and $\left|\Omega_{n}^{+}\right|=\left|\Omega^{+}\right|$, we have

$$
\liminf _{n \rightarrow \infty} F_{\delta_{n}}\left(\mathbf{u}_{n}, \Omega_{n}\right) \geq F_{\infty}(\mathbf{u}, \Omega) .
$$

Indeed by (1.8) and (1.9) it is clear that $\mathbf{E}_{\delta_{n}} \rightarrow \mathbf{E}_{0}$ in $L_{\text {loc }}^{2}\left(\mathbb{R}^{2} ; \mathbb{R}^{2 \times 2}\right)$ and thus, as in (2.27),

$$
\liminf _{n \rightarrow \infty} \int_{\Omega_{n}} W\left(\mathbf{E}\left(\mathbf{u}_{n}\right)-\mathbf{E}_{\delta_{n}}\right) d \mathbf{x} \geq \int_{\Omega} W\left(\mathbf{E}(\mathbf{u})-\mathbf{E}_{0}\right) d \mathbf{x} .
$$

To treat the surface energy we distinguish two cases. If $\gamma_{\text {film }}>\gamma_{\text {sub }}$ then we fix $m \in \mathbb{N}$. Since for all $n \geq m$ we have that $\varphi_{\delta_{n}} \geq \varphi_{\delta_{m}}$ for $y \geq 0$, it follows that

$$
\begin{aligned}
& \liminf _{n \rightarrow \infty} \int_{\Gamma_{n}} \varphi_{\delta_{n}}(y) d \mathcal{H}^{1}(\mathbf{x}) \geq \liminf _{n \rightarrow \infty} \int_{\Gamma_{n}} \varphi_{\delta_{m}}(y) d \mathcal{H}^{1}(\mathbf{x}) \\
& \geq \int_{\widetilde{\Gamma}} \varphi_{\delta_{m}}(y) d \mathcal{H}^{1}(\mathbf{x})+2 \sum_{x \in S} \int_{h(x)}^{h^{-}(x)} \varphi_{\delta_{m}}(y) d y,
\end{aligned}
$$


where the last inequality can be proved as in (2.26). As $\varphi_{\delta_{m}} \nearrow \tilde{\varphi}$, by letting $m \rightarrow \infty$ and using the Lebesgue Monotone Convergence Theorem we conclude that

$$
\liminf _{n \rightarrow \infty} \int_{\Gamma_{n}} \varphi_{\delta_{n}}(y) d \mathcal{H}^{1}(\mathbf{x}) \geq \int_{\widetilde{\Gamma}} \tilde{\varphi}(y) d \mathcal{H}^{1}(\mathbf{x})+2 \sum_{x \in S} \int_{h(x)}^{h^{-}(x)} \tilde{\varphi}(y) d y,
$$

which, together with (2.36) and (2.39), yields (2.38).

The case $\gamma_{\text {film }} \leq \gamma_{\text {sub }}$ is simpler, since by definition we have that $\varphi_{\delta_{n}} \geq \gamma_{\text {film }}$.

Step 2: In view of the previous theorem, in order to prove the estimate from above it is enough to show that for all sequences $\delta_{n} \searrow 0$ and for all $(\mathbf{u}, \Omega)$ in $X$ there exists $\left(\mathbf{u}_{n}, \Omega_{n}\right) \rightarrow(\mathbf{u}, \Omega)$ in $X$ such that

$$
\limsup _{n \rightarrow \infty} \bar{F}_{\delta_{n}}\left(\mathbf{u}_{n}, \Omega_{n}\right) \leq F_{\infty}(\mathbf{u}, \Omega)
$$

where the functionals $\bar{F}_{\delta_{n}}$ are the relaxed functionals given by (2.18).

Fix a sequence $\delta_{n} \searrow 0$ and $(\mathbf{u}, \Omega) \in X$. If $\gamma_{\text {film }}<\gamma_{\text {sub }}$ then construct $\varepsilon_{n} \searrow 0$ such that

$$
\varphi_{\delta_{n}}\left(y+\varepsilon_{n}\right) \rightarrow \tilde{\varphi}(y)=\gamma_{\mathrm{film}}
$$

for all $y \geq 0$. It is well-known that $\mathcal{L}^{1}$-a.e. $y<0$ the function $\mathbf{u}(\cdot, y) \in H^{1}\left((a, b) ; \mathbb{R}^{2}\right)$. Let $y_{0}$ be any such $y$ and define

$$
\mathbf{u}_{n}(\mathbf{x}):= \begin{cases}\mathbf{u}\left(x, y-\varepsilon_{n}\right) & \text { if } y>y_{0}+\varepsilon_{n} \\ \mathbf{u}\left(x, y_{0}\right) & \text { if } y_{0}<y \leq y_{0}+\varepsilon_{n} \\ \mathbf{u}(x, y) & \text { if } y \leq y_{0}\end{cases}
$$

and

$$
h_{n}(x):=\min \left\{h(x)+\varepsilon_{n}, t_{n}\right\}
$$

where $t_{n}>0$ is chosen so that $\left|\Omega_{n}^{+}\right|=d$. Since $\varepsilon_{n} \rightarrow 0$ it follows that $t_{n} \rightarrow \sup h$ and, in turn, by $(2.41)$

$$
\varphi_{\delta_{n}}\left(\min \left\{y+\varepsilon_{n}, t_{n}\right\}\right) \rightarrow \gamma_{\text {film }}
$$

for all $0 \leq y \leq \sup h$. Clearly

$$
\int_{\Omega_{n}} W\left(\mathbf{E}\left(\mathbf{u}_{n}\right)-\mathbf{E}_{\delta_{n}}\right) d \mathbf{x} \rightarrow \int_{\Omega} W\left(\mathbf{E}(\mathbf{u})-\mathbf{E}_{0}\right) d \mathbf{x} .
$$

Moreover,

$$
\begin{aligned}
& \int_{\widetilde{\Gamma}_{n}} \varphi_{\delta_{n}}(y) d \mathcal{H}^{1}(\mathbf{x})+2 \sum_{h_{n}(x) \neq h_{n}^{-}(x)} \int_{h_{n}(x)}^{h_{n}^{-}(x)} \varphi_{\delta_{n}}(y) d y \\
& \leq \int_{\widetilde{\Gamma}} \varphi_{\delta_{n}}\left(\min \left\{y+\varepsilon_{n}, t_{n}\right\}\right) d \mathcal{H}^{1}(\mathbf{x})+2 \sum_{x \in S} \int_{h(x)}^{h^{-}(x)} \varphi_{\delta_{n}}\left(\min \left\{y+\varepsilon_{n}, t_{n}\right\}\right) d y=: A_{n} .
\end{aligned}
$$

Note that by $(2.42),(2.36)$, and the Dominated Convergence Theorem we have

$$
A_{n} \rightarrow \gamma_{\text {film }} \mathcal{H}^{1}(\widetilde{\Gamma})+2 \gamma_{\text {film }} \sum_{x \in S}\left(h^{-}(x)-h(x)\right)
$$

Hence (2.40) follows from $(2.43),(2.44)$, and (2.45).

The case $\gamma_{\text {film }} \geq \gamma_{\text {sub }}$ is simpler, since it is enough to take by $\mathbf{u}_{n}:=\mathbf{u}$ and $\Omega_{n}=\Omega$.

The following compactness result is an easy consequence of Proposition 2.2. 
Proposition 2.10 Let $\left\{\left(\mathbf{u}_{n}, \Omega_{n}\right)\right\} \subset X$ and assume that

$$
\sup _{n}\left(F_{\infty}\left(\mathbf{u}_{n}, \Omega_{n}\right)+\left|\Omega_{n}^{+}\right|\right)<\infty .
$$

Then there exist a subsequence $\left\{\left(\mathbf{u}_{n_{k}}, \Omega_{n_{k}}\right)\right\}$, infinitesimal rigid motions $\mathbf{v}_{k}$, and $(\mathbf{u}, \Omega) \in X$ such that

$$
\left(\mathbf{u}_{n_{k}}+\mathbf{v}_{k}, \Omega_{n_{k}}\right) \rightarrow(\mathbf{u}, \Omega) \text { in } X .
$$

Proof. It is enough to observe that (2.46) implies

$$
\sup _{n}\left(\int_{\Omega_{n}}\left|\mathbf{E}\left(\mathbf{u}_{n}\right)\right|^{2} d \mathbf{x}+\mathcal{H}^{1}\left(\Gamma_{n}\right)+\left|\Omega_{n}^{+}\right|\right)<\infty
$$

and so we can proceed as in the proof of Proposition 2.2.

By the previous proposition, Lemma 2.5 , and the sequential lower semicontinuity of $F_{\infty}$ we obtain immediately:

Corollary 2.11 For every $d>0$ the minimization problem

$$
\min \left\{F_{\infty}(\mathbf{u}, \Omega):(\mathbf{u}, \Omega) \in X, \quad\left|\Omega^{+}\right|=d\right\}
$$

admits a solution.

\section{Regularity of local minimizers}

In this section we study the regularity of minimizers of the limiting problem away from cusp points and cuts in the wetting regime $\gamma_{\text {film }}<\gamma_{\text {sub }}$. We recall that in this case the energy is given by

$$
F_{\infty}(\mathbf{u}, \Omega)=\int_{\Omega} W\left(\mathbf{E}(\mathbf{u})-\mathbf{E}_{0}\right) d \mathbf{x}+\gamma_{\text {film }} \mathcal{H}^{1}(\widetilde{\Gamma})+2 \gamma_{\text {film }} \sum_{x \in S}\left(h^{-}(x)-h(x)\right)
$$

for all $(\mathbf{u}, \Omega) \in X$ (see (2.8)), where $\widetilde{\Gamma}$ and $S$ are the sets defined in (2.4) and (2.17), respectively.

We say that $(\mathbf{u}, \Omega) \in X$ is a $\delta$-local minimizer for the functional $F_{\infty}$ if $F_{\infty}(\mathbf{u}, \Omega)<\infty$ and there exists $\delta>0$ such that

$$
F_{\infty}(\mathbf{u}, \Omega) \leq F_{\infty}\left(\mathbf{v}, \Omega_{g}\right)
$$

for all $\left(\mathbf{v}, \Omega_{g}\right) \in X$ satisfying

$$
\left|\Omega_{g}^{+}\right|=\left|\Omega^{+}\right| \quad \text { and } \quad\left|\Omega_{g} \Delta \Omega\right| \leq \delta .
$$

Note that if $h$ and $g$ are the profile functions associated with $\Omega$ and $\Omega_{g}$, respectively, then condition (3.2) is equivalent to

$$
\int_{a}^{b} g(x) d x=\int_{a}^{b} h(x) d x \text { and } \int_{a}^{b}|h(x)-g(x)| d x \leq \delta .
$$

In order to study the regularity of $(\mathbf{u}, \Omega)$ it is convenient to replace the volume constraint with a suitable volume penalization. This is made precise in the following proposition.

Proposition 3.1 Let $(\mathbf{u}, \Omega) \in X$ be a $\delta$-local minimizer for the functional $F_{\infty}$ and let $d:=\left|\Omega^{+}\right|$. Then there exists $\ell_{0}>0$ such that

$$
F_{\infty}(\mathbf{u}, \Omega)=\min \left\{F_{\infty}\left(\mathbf{v}, \Omega_{g}\right)+\ell|d-| \Omega_{g}^{+}||:\left(\mathbf{v}, \Omega_{g}\right) \in X,\left|\Omega_{g} \Delta \Omega\right| \leq \frac{\delta}{2}\right\}
$$

for all $\ell \geq \ell_{0}$. 
In order to prove the proposition we need the following lemma about the structure of superlevel sets of lower semicontinuous functions with finite pointwise variation.

Lemma 3.2 For $d>0$ set $\tau_{0}:=\frac{d}{4(b-a)}$ and fix $M_{1}>0$. Then there exists a constant $r_{0}=$ $r_{0}\left(d, M_{1}\right)>0$ with the following property: For every lower semicontinuous function $g:[a, b] \rightarrow$ $[0,+\infty)$ such that

$$
\frac{d}{2} \leq \int_{a}^{b} g(x) d x \leq \frac{3}{2} d \quad \text { and } \quad \mathcal{H}^{1}\left(\Gamma_{g}\right) \leq M_{1}
$$

the open set $\left\{x \in(a, b): g(x)>\tau_{0}\right\}$ contains at least one connected component, say $\left(a^{\prime}, b^{\prime}\right)$, such that $b^{\prime}-a^{\prime} \geq r_{0}$.

Proof. Assume by contradiction that there exists a sequence of lower semicontinuous functions $g_{n}:[a, b] \rightarrow[0,+\infty)$, with

$$
\frac{d}{2} \leq \int_{a}^{b} g_{n}(x) d x \leq \frac{3}{2} d \quad \text { and } \quad \mathcal{H}^{1}\left(\Gamma_{g_{n}}\right) \leq M_{1}
$$

such that

$$
|I| \leq \frac{1}{n} \quad \text { for every interval } I \subset\left\{x \in(a, b): g_{n}(x)>\tau_{0}\right\} .
$$

Note that (3.4) implies the existence of a constant $M_{2}>0$ such that

$$
\left\|g_{n}\right\|_{\infty} \leq M_{2} \text { for every } n \text {. }
$$

Set $\tau_{1}:=\frac{3}{8} \frac{d}{(b-a)}$ and consider the family $I_{1}^{n}, \ldots, I_{k_{n}}^{n}$ of all connected components of $\{x \in(a, b)$ : $\left.g_{n}(x)>\tau_{0}\right\}$ having nonempty intersection with $\left\{x \in(a, b): g_{n}(x)>\tau_{1}\right\}$. Since, by definition, we have $\mathcal{H}^{1}\left(\Gamma_{g_{n}} \cap\left(\overline{I_{j}^{n}} \times \mathbb{R}\right)\right)>2\left(\tau_{1}-\tau_{0}\right)$, summing over $j=1, \ldots, k_{n}$ we obtain

$$
2 M_{1} \geq 2 \mathcal{H}^{1}\left(\Gamma_{g_{n}}\right) \geq \sum_{j=1}^{k_{n}} \mathcal{H}^{1}\left(\Gamma_{g_{n}} \cap\left(\overline{I_{j}^{n}} \times \mathbb{R}\right)\right) \geq 2 k_{n}\left(\tau_{1}-\tau_{0}\right),
$$

which implies

$$
k_{n} \leq \frac{M_{1}}{\tau_{1}-\tau_{0}} .
$$

By $(3.5),(3.6)$, and (3.7) we deduce that

$$
\int_{\left\{g_{n}>\tau_{1}\right\}} g_{n} d x \leq \sum_{j=1}^{k_{n}} \int_{I_{j}^{n}} g_{n} d x \leq\left\|g_{n}\right\|_{\infty} \sum_{j=1}^{k_{n}}\left|I_{j}^{n}\right| \leq \frac{M_{1} M_{2}}{n\left(\tau_{1}-\tau_{0}\right)} \rightarrow 0 .
$$

Therefore, using also the definition of $\tau_{1}$, we have

$$
\frac{d}{2} \leq \limsup _{n \rightarrow \infty} \int_{a}^{b} g_{n}(x) d x=\limsup _{n \rightarrow \infty} \int_{\left\{g_{n} \leq \tau_{1}\right\}} g_{n} d x \leq \tau_{1}(b-a)=\frac{3}{8} d
$$

which is a contradiction and concludes the proof of the lemma.

Proof of Proposition 3.1. Arguing as in the previous section (see Corollary 2.11), for any $\ell$ the minimization problem defined on the right-hand side of (3.3) admits a solution $\left(\mathbf{v}_{\ell}, \Omega_{g_{\ell}}\right) \in X$ with

$$
|d-| \Omega_{g_{\ell}}^{+}|| \leq \frac{\delta}{2}
$$

From the minimality of $\mathbf{v}_{\ell}$ we have

$$
\ell|d-| \Omega_{g_{\ell}}^{+}|| \leq F_{\infty}\left(\mathbf{v}_{\ell}, \Omega_{g_{\ell}}\right)+\ell|d-| \Omega_{g_{\ell}}^{+}|| \leq F_{\infty}(\mathbf{u}, \Omega) .
$$


Setting

$$
\ell^{\prime}:=\frac{2 F_{\infty}(\mathbf{u}, \Omega)}{\min \{d, \delta\}} \quad \text { and } \quad M_{1}:=\frac{F_{\infty}(\mathbf{u}, \Omega)}{\gamma_{\text {film }}}
$$

(3.9) and (3.10) immediately yield

$$
\frac{d}{2} \leq \int_{a}^{b} g_{\ell}(x) d x \leq \frac{3}{2} d, \quad \text { and } \quad \mathcal{H}^{1}\left(\Gamma_{g_{\ell}}\right) \leq M_{1}
$$

for $\ell \geq \ell^{\prime}$.

Note that (3.3) holds if we show that

$$
\left|\Omega_{g_{\ell}}^{+}\right|=d
$$

for all $\ell$ sufficiently large. We divide the proof of this fact into two steps.

Step 1: We claim that there exists $\ell_{0}>0$ such that

$$
\left|\Omega_{g_{\ell}}^{+}\right| \geq d \quad \text { for } \ell \geq \ell_{0}
$$

We assume that

$$
\left|\Omega_{g_{\ell}}^{+}\right|<d
$$

for some $\ell>\ell^{\prime}$ (see (3.10) ) and we will prove that this inequality leads to a contradiction if $\ell$ is large enough.

Let $\tau_{0}$ and $r_{0}$ be as in Lemma 3.2. Then by (3.11) and Lemma 3.2 there exists a connected component $I_{\ell}$ of the open set $\left\{x \in(a, b): g_{\ell}(x)>\tau_{0}\right\}$ such that

$$
\left|I_{\ell}\right| \geq r_{0}
$$

For every $\tau \in\left[\tau_{0} / 2, \tau_{0}\right]$ let $\left(a_{\ell \tau}, b_{\ell \tau}\right)$ be the connected component of $\left\{x \in(a, b): g_{\ell}(x)>\tau\right\}$ containing the interval $I_{\ell}$ (in particular, $I_{\ell}=\left(a_{\ell \tau_{0}}, b_{\ell \tau_{0}}\right)$ ). Note that for $0 \leq \tau_{1}<\tau_{2} \leq \tau_{0}$ we have $\left(a_{\ell \tau_{2}}, b_{\ell \tau_{2}}\right) \subset\left(a_{\ell \tau_{1}}, b_{\ell \tau_{1}}\right)$. It easily follows that the set

$$
T_{\ell}:=\left\{(x, \tau) \in(a, b) \times \mathbb{R}: \tau \in\left[\frac{\tau_{0}}{2}, \tau_{0}\right), x \in\left(a_{\ell \tau}, b_{\ell \tau}\right)\right\} \cup\left(\left(a_{\ell \frac{\tau_{0}}{2}}, b_{\ell} \frac{\tau_{0}}{2}\right) \times\left(0, \frac{\tau_{0}}{2}\right)\right)
$$

is a generalized trapezoid, as defined in the Appendix, with parallel sides $s_{1 \ell}, s_{2 \ell}$ of length $l_{1 \ell}:=\left|I_{\ell}\right|$, $l_{2 \ell}:=b_{\ell \frac{\tau_{0}}{2}}-a_{\ell \frac{\tau_{0}}{2}}$ respectively, and height $\tau_{0}$. Note that the non-degeneracy condition (4.7) is satisfied with $\alpha=\frac{\pi}{2}$ and $r_{1 \ell}=\min \left\{\frac{\tau_{0}}{2}, l_{2 \ell}\right\}$. Moreover, taking into account (3.14) we have,

$$
m_{1}:=\frac{1}{2(b-a)} \min \left\{\tau_{0}, r_{0}\right\} \leq \min \left\{\frac{l_{1 \ell}}{l_{2 \ell}}, \frac{r_{1 \ell}}{l_{2 \ell}}, \frac{\tau_{0}}{l_{2 \ell}}\right\} \leq \max \left\{\frac{l_{1 \ell}}{l_{2 \ell}}, \frac{r_{1 \ell}}{l_{2 \ell}}, \frac{\tau_{0}}{l_{2 \ell}}\right\} \leq \frac{1}{r_{0}} \max \left\{\tau_{0}, r_{0}\right\}=: m_{2} .
$$

Denoting by $\mathbf{c}_{\ell}$ the center of $T_{\ell}$ (see the Appendix for its the definition) and by $B_{\ell}$ the ball centered at $\mathbf{c}_{\ell}$ with radius $m_{1} l_{2 \ell} / 2$, it follows from Theorem 4.3 (see also Corollary 4.5) and from (1.4) that there exist positive constants $c_{1}, c_{2}$ independent of $\ell \geq \ell^{\prime}$ such that

$$
\int_{T_{\ell}}\left|\nabla \mathbf{z}_{\ell}\right|^{2} d \mathbf{x} \leq c_{1} \int_{T_{\ell}} W\left(\mathbf{E}\left(\mathbf{z}_{\ell}\right)\right) d \mathbf{x} \leq c_{2}\left(\int_{T_{\ell}} W\left(\mathbf{E}\left(\mathbf{z}_{\ell}\right)-\mathbf{E}_{0}\right) d \mathbf{x}+1\right),
$$

where

$$
\mathbf{z}_{\ell}(\mathbf{x}):=\mathbf{v}_{\ell}(\mathbf{x})+\mathbf{A}_{\ell} \mathbf{x}
$$

with

$$
\mathbf{A}_{\ell}:=\frac{1}{\left|B_{\ell}\right|} \int_{B_{\ell}}\left(\left(\nabla \mathbf{v}_{\ell}\right)^{T}-\nabla \mathbf{v}_{\ell}\right) d \mathbf{x}
$$


Using (3.15), (3.9), and the fact that $\mathbf{E}\left(\mathbf{z}_{\ell}\right)=\mathbf{E}\left(\mathbf{v}_{\ell}\right)$ we obtain

$$
\int_{T_{\ell}}\left|\nabla \mathbf{z}_{\ell}\right|^{2} d \mathbf{x} \leq c_{2}\left(F_{\infty}(\mathbf{u}, \Omega)+1\right) \quad \text { for } \ell \geq \ell^{\prime}
$$

Define

$$
\eta_{\ell}:=\frac{d-\left|\Omega_{g_{\ell}}^{+}\right|}{r_{0}}
$$

and divide the interval $\left[\tau_{0} / 2, \tau_{0}\right]$ in $k_{\ell}$ intervals with length between $\eta_{\ell}$ and $2 \eta_{\ell}$, where

$$
k_{\ell}:=\left[\frac{\tau_{0}}{2 \eta_{\ell}}\right] .
$$

From (3.17) it is clear that at least one of these intervals, say $\left(\tau_{\ell}^{\prime}, \tau_{\ell}^{\prime \prime}\right)$, satisfies

$$
\int_{\left(a_{\ell \tau_{\ell}^{\prime \prime}}, b_{\ell \tau_{\ell}^{\prime \prime}}\right) \times\left(\tau_{\ell}^{\prime}, \tau_{\ell}^{\prime \prime}\right)}\left|\nabla \mathbf{z}_{\ell}\right|^{2} d \mathbf{x} \leq \int_{T_{\ell} \cap\left((a, b) \times\left(\tau_{\ell}^{\prime}, \tau_{\ell}^{\prime \prime}\right)\right)}\left|\nabla \mathbf{z}_{\ell}\right|^{2} d \mathbf{x} \leq \frac{c_{2}}{k_{\ell}}\left(F_{\infty}(\mathbf{u}, \Omega)+1\right) .
$$

For simplicity, from now on we write $\left(a_{\ell}, b_{\ell}\right)$ in place of $\left(a_{\ell \tau_{\ell}^{\prime \prime}}, b_{\ell \tau_{\ell}^{\prime \prime}}\right)$. We define

$$
h_{\ell}(x):= \begin{cases}g_{\ell}(x) & \text { if } x \in(a, b) \backslash\left(a_{\ell}, b_{\ell}\right), \\ g_{\ell}(x)+\bar{\eta}_{\ell} & \text { if } x \in\left(a_{\ell}, b_{\ell}\right),\end{cases}
$$

where

$$
\bar{\eta}_{\ell}:=\frac{d-\left|\Omega_{g_{\ell}}^{+}\right|}{b_{\ell}-a_{\ell}} \leq \eta_{\ell}
$$

and

$$
\mathbf{w}_{\ell}(x, y):= \begin{cases}\mathbf{z}_{\ell}(x, y) & \text { if } x \in(a, b) \backslash\left(a_{\ell}, b_{\ell}\right) \text { or } y<\tau_{\ell}^{\prime \prime} \\ \hat{\mathbf{z}}_{\ell}(x, y) & \text { if } x \in\left(a_{\ell}, b_{\ell}\right) \text { and } \tau_{\ell}^{\prime \prime}<y<\tau_{\ell}^{\prime \prime}+\frac{\bar{\eta}_{\ell}}{2} \\ \mathbf{z}_{\ell}\left(x, y-\bar{\eta}_{\ell}\right) & \text { if } x \in\left(a_{\ell}, b_{\ell}\right) \text { and } \tau_{\ell}^{\prime \prime}+\frac{\bar{\eta}_{\ell}}{2}<y<h_{\ell}(x)\end{cases}
$$

where

$$
\hat{\mathbf{z}}_{\ell}(x, y):=\mathbf{z}_{\ell}\left(x, 2 \tau_{\ell}^{\prime \prime}-y\right) .
$$

By construction $\int_{a}^{b} h_{\ell}(x) d x=d$. Moreover, using (3.8) we have

$$
\int_{a}^{b}\left|h(x)-h_{\ell}(x)\right| d x \leq \int_{a}^{b}\left|h(x)-g_{\ell}(x)\right| d x+\bar{\eta}_{\ell}\left(b_{\ell}-a_{\ell}\right) \leq \frac{\delta}{2}+d-\left|\Omega_{g_{\ell}}^{+}\right| \leq \delta
$$

for $\ell \geq \ell^{\prime}$. Since $(\mathbf{u}, \Omega)$ is $\delta$-local minimizer it follows that

$$
F_{\infty}(\mathbf{u}, \Omega) \leq F_{\infty}\left(\mathbf{w}_{\ell}, \Omega_{h_{\ell}}\right)
$$

By (1.3) there exists a constant $c_{3}>0$ independent of $\ell$ such that

$$
W\left(\mathbf{E}\left(\mathbf{z}_{\ell}\right)-\mathbf{E}_{0}\right) \leq c_{3}\left(\left|\nabla \mathbf{z}_{\ell}\right|^{2}+1\right) \quad \text { in }\left(a_{\ell}, b_{\ell}\right) \times\left(\tau_{\ell}^{\prime}, \tau_{\ell}^{\prime \prime}\right)
$$

and

$$
W\left(\mathbf{E}\left(\hat{\mathbf{z}}_{\ell}\right)-\mathbf{E}_{0}\right) \leq c_{3}\left(\left|\nabla \hat{\mathbf{z}}_{\ell}\right|^{2}+1\right) \quad \text { in }\left(a_{\ell}, b_{\ell}\right) \times\left(\tau_{\ell}^{\prime \prime}, \tau_{\ell}^{\prime \prime}+\frac{\bar{\eta}_{\ell}}{2}\right), .
$$

Using $(3.20),(3.23)$, and (3.24) we easily obtain

$$
\int_{\left(a_{\ell}, b_{\ell}\right) \times\left(\tau_{\ell}^{\prime}, \tau_{\ell}^{\prime \prime}\right)} W\left(\mathbf{E}\left(\mathbf{z}_{\ell}\right)-\mathbf{E}_{0}\right) d \mathbf{x} \leq c_{3} \int_{\left(a_{\ell}, b_{\ell}\right) \times\left(\tau_{\ell}^{\prime}, \tau_{\ell}^{\prime \prime}\right)}\left(\left|\nabla \mathbf{z}_{\ell}\right|^{2}+1\right) d \mathbf{x} \leq \frac{c_{4}}{k_{\ell}}\left(F_{\infty}(\mathbf{u}, \Omega)+1\right)
$$


and

$$
\begin{aligned}
\int_{\left(a_{\ell}, b_{\ell}\right) \times\left(\tau_{\ell}^{\prime \prime}, \tau_{\ell}^{\prime \prime}+\frac{\bar{\eta}_{\ell}}{2}\right)} W\left(\mathbf{E}\left(\hat{\mathbf{z}}_{\ell}\right)-\mathbf{E}_{0}\right) d \mathbf{x} & \leq c_{3} \int_{\left(a_{\ell}, b_{\ell}\right) \times\left(\tau_{\ell}^{\prime \prime}, \tau_{\ell}^{\prime \prime}+\overline{\bar{\eta}}_{\ell}\right)}\left(\left|\nabla \hat{\mathbf{z}}_{\ell}\right|^{2}+1\right) d \mathbf{x} \\
& \leq \frac{c_{4}}{k_{\ell}}\left(F_{\infty}(\mathbf{u}, \Omega)+1\right),
\end{aligned}
$$

for a suitable constant $c_{4}>0$ still independent of $\ell \geq \ell^{\prime}$. Note that, setting

$$
\ell^{\prime \prime}:=\max \left\{\frac{4 F_{\infty}(\mathbf{u}, \Omega)}{\tau_{0} r_{0}}, \ell^{\prime}\right\},
$$

by (3.9) and (3.21) we have $2 /\left(\tau_{0}-2 \eta_{\ell}\right) \leq 4 / \tau_{0}$ for $\ell \geq \ell^{\prime \prime}$, which, by (3.19), implies

$$
\frac{1}{k_{\ell}} \leq \frac{2 \eta_{\ell}}{\tau_{0}-2 \eta_{\ell}}=\frac{d-\left|\Omega_{g_{\ell}}^{+}\right|}{r_{0}} \frac{2}{\tau_{0}-2 \eta_{\ell}} \leq \frac{4\left(d-\left|\Omega_{g_{\ell}}^{+}\right|\right)}{r_{0} \tau_{0}} .
$$

Using also (3.18), (3.21), (3.25), (3.26), and the fact that $F_{\infty}\left(\mathbf{v}_{\ell}, \Omega_{g_{\ell}}\right)=F_{\infty}\left(\mathbf{z}_{\ell}, \Omega_{g_{\ell}}\right)$ (see (3.16)), for $\ell \geq \ell^{\prime \prime}$ we deduce that

$$
\begin{aligned}
F_{\infty}\left(\mathbf{w}_{\ell}, \Omega_{h_{\ell}}\right)= & F_{\infty}\left(\mathbf{v}_{\ell}, \Omega_{g_{\ell}}\right)+\int_{\left(a_{\ell}, b_{\ell}\right) \times\left(\tau_{\ell}^{\prime \prime}, \tau_{\ell}^{\prime \prime}+\frac{\bar{\eta}_{\ell}}{2}\right)} W\left(\mathbf{E}\left(\hat{\mathbf{z}}_{\ell}\right)-\mathbf{E}_{0}\right) d \mathbf{x} \\
& +\int_{\left(a_{\ell}, b_{\ell}\right) \times\left(\tau_{\ell}^{\prime}, \tau_{\ell}^{\prime}+\frac{\bar{\eta}_{\ell}}{2}\right)} W\left(\mathbf{E}\left(\mathbf{z}_{\ell}\right)-\mathbf{E}_{0}\right) d \mathbf{x}+2 \gamma_{\mathrm{film}} \bar{\eta}_{\ell} \\
\leq & F_{\infty}\left(\mathbf{v}_{\ell}, \Omega_{g_{\ell}}\right)+\frac{2 c_{4}}{k_{\ell}}\left(F_{\infty}(\mathbf{u}, \Omega)+1\right)+2 \gamma_{\mathrm{film}} \eta_{\ell} \\
\leq & F_{\infty}\left(\mathbf{v}_{\ell}, \Omega_{g_{\ell}}\right)+\frac{d-\left|\Omega_{g_{\ell}}^{+}\right|}{r_{0}}\left(\frac{8 c_{4}}{\tau_{0}}\left(F_{\infty}(\mathbf{u}, \Omega)+1\right)+2 \gamma_{\mathrm{film}}\right) .
\end{aligned}
$$

Thus, if

$$
\ell \geq \ell_{0}:=\max \left\{\frac{1}{r_{0}}\left(\frac{8 c_{4}}{\tau_{0}}\left(F_{\infty}(\mathbf{u}, \Omega)+1\right)+2 \gamma_{\text {film }}\right), \ell^{\prime \prime}\right\}+1,
$$

and recalling (3.9), then it follows that

$$
F_{\infty}\left(\mathbf{w}_{\ell}, \Omega_{h_{\ell}}\right)<F_{\infty}\left(\mathbf{v}_{\ell}, \Omega_{g_{\ell}}\right)+\ell\left(d-\left|\Omega_{g_{\ell}}^{+}\right|\right) \leq F_{\infty}(\mathbf{u}, \Omega),
$$

which contradicts (3.22). This shows that (3.13) holds if $\ell \geq \ell_{0}$.

Step 2: To show (3.12), by the previous step, it suffices to exclude the case

$$
\left|\Omega_{g_{\ell}}^{+}\right|>d
$$

when $\ell>\ell_{0}$ (see (3.27) ). If (3.28) holds then we can find $t_{\ell}>0$ such that $\left|\Omega_{h_{\ell}}^{+}\right|=d$, where

$$
h_{\ell}:=\min \left\{g_{\ell}, t_{\ell}\right\} .
$$

Note that if $\ell>\ell_{0}$, by (3.8) we have

$$
\begin{aligned}
\int_{a}^{b}\left|h(x)-h_{\ell}(x)\right| d x & \leq \int_{a}^{b}\left|h(x)-g_{\ell}(x)\right| d x+\int_{a}^{b}\left(g_{\ell}(x)-h_{\ell}(x)\right) d x \\
& \leq \frac{\delta}{2}+\left|\Omega_{g_{\ell}}^{+}\right|-d \leq \delta .
\end{aligned}
$$


Hence the pair $\left(\mathbf{v}_{\ell}, \Omega_{h_{\ell}}\right)$ is admissible for the volume constrained minimization problem. On the other hand

$$
\begin{aligned}
\gamma_{\text {film }} \mathcal{H}^{1}\left(\widetilde{\Gamma}_{h_{\ell}}\right) & +2 \gamma_{\text {film }} \sum_{h_{\ell}(x) \neq h_{\ell}^{-}(x)}\left(h_{\ell}^{-}(x)-h_{\ell}(x)\right) \\
& <\gamma_{\text {film }} \mathcal{H}^{1}\left(\widetilde{\Gamma}_{g_{\ell}}\right)+2 \gamma_{\text {film }} \sum_{g_{\ell}(x) \neq g_{\ell}^{-}(x)}\left(g_{\ell}^{-}(x)-g_{\ell}(x)\right),
\end{aligned}
$$

which implies, taking into account also (3.9),

$$
F_{\infty}\left(\mathbf{v}_{\ell}, \Omega_{h_{\ell}}\right)<F_{\infty}\left(\mathbf{v}_{\ell}, \Omega_{g_{\ell}}\right)<F_{\infty}\left(\mathbf{v}_{\ell}, \Omega_{g_{\ell}}\right)+\ell\left(\left|\Omega_{g_{\ell}}^{+}\right|-d\right) \leq F_{\infty}(\mathbf{u}, \Omega)
$$

The last chain of inequalities contradicts the local minimality of $(\mathbf{u}, \Omega)$ and concludes the proof of the proposition.

Following an idea of Chambolle and Larsen in [7] we begin by establishing an internal sphere condition.

Proposition 3.3 (Internal sphere) Let $(\mathbf{u}, \Omega) \in X$ be a $\delta$-local minimizer for the functional $F_{\infty}$. Then, for every $\mathbf{z}_{0} \in \bar{\Gamma}$ there exists an open ball $B\left(\mathbf{x}_{0}, \rho_{0}\right)$, with $\rho_{0}$ independent of $\mathbf{z}_{0}$ and with $B\left(\mathbf{x}_{0}, \rho_{0}\right) \cap((a, b) \times \mathbb{R}) \subset \Omega$, such that

$$
\partial B\left(\mathbf{x}_{0}, \rho_{0}\right) \cap \bar{\Gamma}=\left\{\mathbf{z}_{0}\right\} .
$$

Proof. We divide the proof into two steps.

Step 1: We first prove that there exists $\rho_{0}>0$ such that for any (open) ball $B\left(\mathbf{x}_{0}, \rho_{0}\right)$, with

$$
B\left(\mathbf{x}_{0}, \rho_{0}\right) \cap((a, b) \times \mathbb{R}) \subset \Omega,
$$

the set $\partial B\left(\mathbf{x}_{0}, \rho_{0}\right)$ intersects $\bar{\Gamma}$ in at most one point.

By the previous proposition there exists $\ell_{0}>0$ such that

$$
F_{\infty}(\mathbf{u}, \Omega)=\min \left\{H_{\infty}\left(\mathbf{v}, \Omega_{g}\right):\left(\mathbf{v}, \Omega_{g}\right) \in X,\left|\Omega_{g} \Delta \Omega\right| \leq \frac{\delta}{2}\right\}
$$

where

$$
H_{\infty}\left(\mathbf{u}, \Omega_{g}\right):=F_{\infty}\left(\mathbf{u}, \Omega_{g}\right)+\ell_{0}|d-| \Omega_{g}^{+}||
$$

and $d:=\left|\Omega^{+}\right|$.

Fix

$$
0<\varepsilon_{0}<\frac{\delta}{4(b-a)}
$$

and choose $\varepsilon<\frac{\varepsilon_{0}}{2}$ and a finite set $A \subset(a, b)$ such that

$$
\sum_{x \in S(h) \backslash A}\left|h\left(x^{+}\right)-h\left(x^{-}\right)\right|+\sum_{x \in S \backslash A}\left(h^{-}(x)-h(x)\right)<\frac{\varepsilon}{2},
$$

where $S(h)$ and $S$ are the sets defined in (2.15) and (2.17), respectively. We also consider the measure $\mu$ obtained by projecting $\mathcal{H}^{1}\lfloor\Gamma$ on the $x$-axis. Since the functions

$$
g_{n}(x):=\mu([x-1 / n, x+1 / n] \backslash(S(h) \cup S))
$$

are upper semicontinuous and $g_{n} \searrow 0$ by a version of the Dini Theorem (see Theorem 7.3 [12]) it follows that $\left\{g_{n}\right\}$ converges to 0 uniformly. Taking into account $(3.32)$, it is easy to see that there exists $r_{0}>0$ satisfying

$$
r_{0}<\min \left\{\left|x-x^{\prime}\right|: x \neq x^{\prime}, x, x^{\prime} \in A\right\}
$$


such that

$$
\sup \left\{\mu(I \backslash A): I \subset(a, b) \text { interval, }|I| \leq r_{0}\right\}<\frac{\varepsilon}{2} .
$$

Let $B\left(\mathbf{x}_{0}, \rho\right)$ be an open ball such that $B\left(\mathbf{x}_{0}, \rho\right) \cap((a, b) \times \mathbb{R}) \subset \Omega$ and assume that the set $\partial B\left(\mathbf{x}_{0}, \rho\right) \cap \bar{\Gamma}$ contains at least two points $\mathbf{c}$ and $\mathbf{d}$. We can write $\mathbf{c}=\left(x_{1}, y_{1}\right)$, with $h\left(x_{1}\right) \leq y_{1} \leq$ $h^{+}\left(x_{1}\right)$, and analogously $\mathbf{d}=\left(x_{2}, y_{2}\right)$, with $h\left(x_{2}\right) \leq y_{2} \leq h^{+}\left(x_{2}\right)$. We set

$$
\Gamma_{\mathbf{c}, \mathbf{d}}:=\left(\widetilde{\Gamma} \cap\left[x_{1}, x_{2}\right] \times \mathbb{R}\right) \cup \bigcup_{i=1}^{2}\left\{\left(x_{i}, y\right): y_{i} \leq y \leq h^{+}\left(x_{i}\right)\right\}
$$

and denote the chord $\{\mathbf{c}+t(\mathbf{d}-\mathbf{c}): 0 \leq t \leq 1\}$ by $[\mathbf{c}, \mathbf{d}]$. Since we will modify $\Omega$ by removing the bounded component $D$ of $\left(\Gamma_{\mathbf{c}, \mathbf{d}} \cup[\mathbf{c}, \mathbf{d}]\right)^{c}$ we need to estimate how much we gain in terms of surface energy. Setting $l:=\mathcal{H}^{1}([\mathbf{c}, \mathbf{d}]), L:=\mathcal{H}^{1}\left(\Gamma_{\mathbf{c}, \mathbf{d}}\right)$, and

$$
r_{1}:=\min \left\{\frac{\varepsilon_{0}}{2}, r_{0}\right\},
$$

we will prove the following claim:

Claim: If

$$
2 \rho<r_{1}
$$

then

$$
\gamma_{\text {film }}(L-l) \leq \ell_{0}|D| \text {. }
$$

We first estimate $L$. Note that by (3.33) the set $\left[x_{1}, x_{2}\right] \cap A$ contains at most one point. Let us consider the worst case: $\left[x_{1}, x_{2}\right] \cap A=\{\bar{x}\}$ and $\sigma:=(\{\bar{x}\} \times \mathbb{R}) \cap \Gamma_{\mathbf{c}, \mathbf{d}}$ has positive $\mathcal{H}^{1}$-measure. Clearly $\sigma$ is a vertical segment and we denote its end-points by $\mathbf{w}_{1}=\left(\bar{x}, y^{\prime}\right)$ and $\mathbf{w}_{2}=\left(\bar{x}, y^{\prime \prime}\right)$. It is also clear that we can write $\Gamma_{\mathbf{c}, \mathbf{d}}$ as a union of $\Gamma_{1}, \sigma$, and $\Gamma_{2}$, where $\Gamma_{1}$ is the (possibly degenerate) subarc connecting $\mathbf{c}$ with $\mathbf{w}_{1}$ while $\Gamma_{2}$ is the subarc connecting $\mathbf{w}_{2}$ with $\mathbf{d}$. As $\mathcal{H}^{1}\left(\Gamma_{1}\right)+\mathcal{H}^{1}\left(\Gamma_{2}\right) \leq$ $2 \mu\left(\left[x_{1}, x_{2}\right] \backslash A\right) \leq \varepsilon$ by $(3.34),(3.35)$, and the fact that $l \leq 2 \rho<r_{1}$, we have

$$
\mathcal{H}^{1}(\sigma) \leq \mathcal{H}^{1}\left(\Gamma_{1}\right)+\mathcal{H}^{1}\left(\Gamma_{2}\right)+l \leq \varepsilon+r_{1}
$$

and, in turn,

$$
L \leq \mathcal{H}^{1}\left(\Gamma_{1}\right)+\mathcal{H}^{1}\left(\Gamma_{2}\right)+\mathcal{H}^{1}(\sigma) \leq 2 \varepsilon+r_{1}
$$

We now define

$$
\tilde{h}(x):= \begin{cases}\frac{y_{2}-y_{1}}{x_{2}-x_{1}}\left(x-x_{1}\right)+y_{1} & \text { if } x_{1}<x<x_{2}, \\ h(x) & \text { otherwise. }\end{cases}
$$

We claim that the pair $\left(\mathbf{u}, \Omega_{\tilde{h}}\right) \in X$ is admissible for (3.29). Indeed by construction $\tilde{h} \leq h$ and

$$
\Omega \backslash \Omega_{\tilde{h}} \subset\left\{(x, y): x_{1}<x<x_{2}, \tilde{h}(x) \leq y \leq h^{+}(x)\right\} \subset \bar{D} .
$$

Hence if $x_{1}<x<x_{2}$ then

$$
h^{+}(x)-\tilde{h}(x) \leq r_{1}+L \leq 2 \varepsilon+2 r_{1} \leq 2 \varepsilon_{0},
$$

where we used (3.35), (3.37), and the fact that $\varepsilon<\frac{\varepsilon_{0}}{2}$. Recalling (3.31), this shows that $\left|\Omega_{\tilde{h}} \Delta \Omega\right| \leq$ $\delta / 2$ and proves the claim.

By (3.29) and (3.3) we then have

$$
F_{\infty}(\mathbf{u}, \Omega)=H_{\infty}(\mathbf{u}, \Omega) \leq H_{\infty}\left(\mathbf{u}, \Omega_{\tilde{h}}\right)
$$

Moreover, it is easy to check that

$$
H_{\infty}\left(\mathbf{u}, \Omega_{\tilde{h}}\right)+\gamma_{\text {film }}(L-l)+\ell_{0}\left(|| \Omega^{+}|-d|-|| \Omega_{\tilde{h}}^{+}|-d|\right) \leq F_{\infty}(\mathbf{u}, \Omega) .
$$


Combining (3.39) and (3.40) we easily get (3.36).

From now on we can proceeds exactly as in the proof of Lemma 1 in [7]. For the reader's convenience we reproduce here the argument. First note that, setting $\theta:=L / l>1$, by the isoperimetric inequality (see [2]) we have

$$
|D| \leq \frac{(l+L)^{2}}{4 \pi}=\frac{(\theta+1)^{2} l^{2}}{4 \pi} .
$$

On the other hand, (3.36) can be written as

$$
l \leq \frac{\ell_{0}|D|}{\gamma_{\text {film }}(\theta-1)} .
$$

Substituting in (3.41) we obtain

$$
|D| \leq \frac{\ell_{0}^{2}}{4 \pi \gamma_{\text {film }}^{2}} \frac{(\theta+1)^{2}}{(\theta-1)^{2}}|D|^{2}
$$

Finally, since by (3.38)

$$
|D|=\int_{x_{1}}^{x_{2}}\left(h^{+}(x)-\tilde{h}(x)\right) d x \leq 2 \varepsilon_{0}(b-a),
$$

from (3.42) we deduce that if $\varepsilon_{0}$ is small enough then $\theta$ must be close to 1 . As the arc of circle of $\partial B\left(\mathbf{x}_{0}, \rho\right)$ which lies above the chord $[\mathbf{c}, \mathbf{d}]$ has length less than or equal to $L$, we conclude that if $\theta<\pi / 2$ then $[\mathbf{c}, \mathbf{d}]$ lies above $\mathbf{x}_{0}$. We may therefore find a point $\mathbf{z}_{0}$ in the segment $\left\{\mathbf{e}+t\left(\mathbf{x}_{0}-\mathbf{e}\right): 0<t \leq 1\right\}$, with $\mathbf{e}$ the middle point of $[\mathbf{c}, \mathbf{d}]$, such that, setting $\bar{\rho}:=\left|\mathbf{z}_{0}-\mathbf{c}\right|$ $=\left|\mathbf{z}_{0}-\mathbf{d}\right|$, the arc of circle of $\partial B\left(\mathbf{z}_{0}, \bar{\rho}\right)$ which lies above the chord $[\mathbf{c}, \mathbf{d}]$ has length equal to $L$. Denote by $2 \alpha<\pi$ the angle associated with such an arc, so that $L=2 \bar{\rho} \alpha$, and let $\mathcal{D}$ be the area enclosed by the same arc and the chord $[\mathbf{c}, \mathbf{d}]$. Note that $\mathcal{D}$ is the largest area bounded by $[\mathbf{c}, \mathbf{d}]$ and a curve of length $L$. Hence $|D| \leq|\mathcal{D}|$. Elementary computations yield $l=2 \bar{\rho} \sin \alpha$ and $|\mathcal{D}|=\left(\bar{\rho}^{2} / 2\right)(2 \alpha-\sin 2 \alpha)$. We deduce from (3.36) that

$$
2 \gamma_{\text {film }} \bar{\rho}(\alpha-\sin \alpha) \leq \frac{\ell_{0} \bar{\rho}^{2}}{2}(2 \alpha-\sin 2 \alpha)
$$

and, in turn,

$$
\rho_{0} \geq \bar{\rho} \geq \frac{4 \gamma_{\mathrm{film}}}{\ell_{0}}\left(\frac{\alpha-\sin \alpha}{2 \alpha-\sin 2 \alpha}\right) \geq \frac{\gamma_{\mathrm{film}}}{2 \ell_{0}},
$$

where the last inequality holds for all $\alpha$. Hence if $\rho_{0}<\gamma_{\text {film }} / 2 \ell_{0}$ is so small that $\theta<\pi / 2$ we have a contradiction. This concludes Step 1.

Step 2: We now deduce from Step 1 the uniform internal sphere conditions stated in the proposition. In this step we essentially reproduce the argument of Lemma 2 in [7]. Consider the union $U$ of all balls of radius $\rho_{0}$ that are contained in $\widetilde{\Omega}:=\Omega \cup[(\mathbb{R} \backslash(a, b)) \times \mathbb{R}]$. It is easy to see that the thesis of the lemma is equivalent to showing that $\Omega \subset U \cap((a, b) \times \mathbb{R})$. Assume by contradiction that such an inclusion doesn't hold. Then there exist $\mathbf{x}_{\mathbf{0}} \in \Omega \cap \partial U$, a sequence of balls $B\left(\mathbf{y}_{n}, \rho_{0}\right) \subset \widetilde{\Omega}$, and $\mathbf{x}_{n} \in \partial B\left(\mathbf{y}_{n}, \rho_{0}\right)$ such that $\mathbf{x}_{n} \rightarrow \mathbf{x}_{0}$. Up to extracting a subsequence (not relabelled) we may assume that $B\left(\mathbf{y}_{n}, \rho_{0}\right) \rightarrow B\left(\mathbf{y}, \rho_{0}\right)$ in the Hausdorff metric, for some ball $B\left(\mathbf{y}, \rho_{0}\right) \subset \widetilde{\Omega}$ having $\mathbf{x}_{0}$ at its boundary. Note that the intersection of $\partial B\left(\mathbf{y}, \rho_{0}\right)$ with $\bar{\Gamma}$ must be nonempty, since if it were we could translate the ball slightly still remaining in $\widetilde{\Omega}$ and this would violate the fact that $\mathbf{x}_{\mathbf{0}} \in \partial U$. Hence, by the previous step, $\partial B\left(\mathbf{y}, \rho_{0}\right) \cap \bar{\Gamma}=\{\mathbf{z}\}$. If $\mathbf{x}_{0}$ and $\mathbf{z}$ are antipodal, then we can find $\delta>0$ such that $B\left(\mathbf{y}+\delta\left(\mathbf{x}_{0}-\mathbf{z}\right), \rho_{0}\right) \subset \widetilde{\Omega}$, which would imply that $\mathbf{x}_{0} \in U$, a contradiction. If $\mathbf{x}_{0}$ and $\mathbf{z}$ are not antipodal, then we can rotate $B\left(\mathbf{y}, \rho_{0}\right)$ around $\mathbf{x}_{0}$, slightly away from $\mathbf{z}$, to get a ball $B^{\prime}$ of radius $\rho_{0}$ such that $\bar{B}^{\prime} \subset \widetilde{\Omega}$ and $\mathbf{x}_{0} \in \partial B^{\prime}$. Translating now $B^{\prime}$ towards $\mathbf{x}_{0}$ we find a ball of the same radius containing $\mathbf{x}_{0}$ and contained in $\widetilde{\Omega}$, which gives again $\mathbf{x}_{0} \in U$. This concludes the proof of the proposition. 
Remark 3.4 Setting $\widetilde{\Omega}:=\Omega \cup[(\mathbb{R} \backslash(a, b)) \times \mathbb{R}]$ we note that Proposition 3.3 can be restated in the following way: There is $\rho_{0}>0$ such that for every $\mathbf{z}_{0} \in \partial \widetilde{\Omega}$ there exists an open ball B $\left(\mathbf{x}_{0}, \rho_{0}\right)$, with $B\left(\mathbf{x}_{0}, \rho_{0}\right) \subset \widetilde{\Omega}$, such that

$$
\partial B\left(\mathbf{x}_{0}, \rho_{0}\right) \cap \partial \widetilde{\Omega}=\left\{\mathbf{z}_{0}\right\}
$$

The next Lipschitz regularity result is a consequence of the uniform sphere condition just proved. Its proof, which relies upon elementary geometrical arguments, is essentially given in [7, Lemma 3]. In the quoted lemma an external uniform condition is assumed, but it can be checked that exactly the same arguments go through in our situation and lead to the following proposition, which we state without proof.

Proposition 3.5 Let $(\mathbf{u}, \Omega) \in X$ be a local minimizer for the functional $F_{\infty}$. Then for any $\mathbf{z}_{0} \in \bar{\Gamma}$ there exist an orthonormal basis $\mathbf{e}_{1}, \mathbf{e}_{2} \in \mathbb{R}^{2}$, and a rectangle

$$
Q:=\left\{\mathbf{z}_{0}+s \mathbf{e}_{1}+t \mathbf{e}_{2}:-a^{\prime}<s<a^{\prime},-b^{\prime}<t<b^{\prime}\right\}
$$

$a^{\prime}, b^{\prime}>0$, such that $\Omega \cap Q$ has one of the following two representations:

(i) There exists a Lipschitz function $g:\left(-a^{\prime}, a^{\prime}\right) \rightarrow\left(-b^{\prime}, b^{\prime}\right)$ such that $g(0)=0$ and

$$
\Omega \cap Q=\left\{\mathbf{z}_{0}+s \mathbf{e}_{1}+t \mathbf{e}_{2}:-a^{\prime}<s<a^{\prime},-b^{\prime}<t<g(s)\right\} \cap((a, b) \times \mathbb{R}) .
$$

Moreover, the function $g$ admits left and right derivatives at every point that are, respectively, left and right continuous.

(ii) There exist two Lipschitz functions $g_{1}, g_{2}:\left[0, a^{\prime}\right) \rightarrow\left(-b^{\prime}, b^{\prime}\right)$ such that $g_{i}(0)=\left(g_{i}\right)_{+}^{\prime}(0)=0$ for $i=1,2, g_{1} \leq g_{2}$, and

$$
\Omega \cap Q=\left\{\mathbf{z}_{0}+s \mathbf{e}_{1}+t \mathbf{e}_{2}: 0<s<a^{\prime},-b^{\prime}<t<g_{1}(s) \text { or } g_{2}(s)<t<b^{\prime}\right\} .
$$

Moreover, the functions $g_{1}, g_{2}$ admit left and right derivatives at every point that are, respectively, left and right continuous.

Remark 3.6 Note that in case (ii) the point $\mathbf{z}_{0}$ is either a cusp point or the lower end-point of a vertical cut (see (2.16)). Proposition 3.5 combined with a simple compactness argument implies that the set of all such points is finite. In particular the set $\Gamma_{\text {cuts }}$ is given by the union of a finite number of vertical cuts. We also remark that the upper end-point of each vertical cut must be a cusp point. We denote by $\Gamma_{\text {cusps }}$ the set of all cusp points; i.e.,

$$
\Gamma_{\text {cusps }}:=\left\{\left(x, h^{-}(x)\right) \text { : either } x \in S(h) \text { or } x \in(a, b) \backslash S(h) \text { and }\left(h^{-}\right)_{+}^{\prime}(x)=-\left(h^{-}\right)_{-}^{\prime}(x)=+\infty\right\} \text {, }
$$

where $S(h)$ is the jump set of $h$ defined in $(2.15)$ and $\left(h^{-}\right)_{+}^{\prime}(x),\left(h^{-}\right)_{-}^{\prime}(x)$ denote the right derivative and the left derivative of $h^{-}$at $x$. We shall also consider as singular points the lower tips of the cuts. This motivates the following notation:

$$
\Gamma_{\text {sing }}:=\Gamma_{\text {cusps }} \cup\{(x, h(x)): x \in S\},
$$

where $S$ is the set defined in (2.17). From the previous observations we have that $\Gamma_{\text {cusps }}$ and $\Gamma_{\text {sing }}$ are finite.

Hereafter we assume that $W$ is the bulk energy density of a linearly isotropic material, i.e.

$$
W(\mathbf{E})=\frac{1}{2} \lambda[\operatorname{tr}(\mathbf{E})]^{2}+\mu \operatorname{tr}\left(\mathbf{E}^{2}\right)
$$

where $\lambda$ and $\mu$ are the (constant) Lamé moduli with

$$
\mu>0, \quad \mu+\lambda>0 .
$$


Note that in this range, the quadratic form $W$ is coercive. Also, the Euler-Lagrange system of equations associated to $W$ is

$$
\mu \Delta \mathbf{u}+(\lambda+\mu) \nabla(\operatorname{div} \mathbf{u})=\mathbf{0} \text { in } \Omega .
$$

We now show that if the boundary is flat then solutions of the Lamé system with natural Neumann boundary conditions are smooth up to the boundary.

Theorem 3.7 Let $\Omega$ be a half-ball of radius one, let $\Omega^{\prime}$ be the concentric half ball of radius $\frac{1}{2}$, and let $\mathbf{u} \in W^{1,2}\left(\Omega ; \mathbb{R}^{2}\right)$ be a weak solution of the Neumann problem

$$
\begin{aligned}
& \mu \Delta \mathbf{u}+(\lambda+\mu) \nabla(\operatorname{div} \mathbf{u})=\mathbf{0} \quad \text { in } \Omega, \\
& {\left[\mu\left(\nabla \mathbf{u}+\nabla \mathbf{u}^{T}\right)+\lambda(\operatorname{div} \mathbf{u}) I\right] \nu=\mathbf{0} \text { on } \Gamma,}
\end{aligned}
$$

where $\Gamma$ is the flat part of $\partial \Omega$. Then for all integers $k \in \mathbb{N}$ there exists a constant $c_{k}>0$ depending only $\lambda$, $\mu$ such that

$$
\sup _{\Omega^{\prime}}\left|\nabla^{k} \mathbf{u}\right|^{2} \leq c_{k} \int_{\Omega}|\nabla \mathbf{u}|^{2} d \mathbf{x} .
$$

Proof. Let $\mathbf{x}_{0}$ be the center of the ball. By Sobolev Embedding Theorem and an easy iteration argument it is enough to show that for all $\frac{1}{2} \leq r<R<1$ and for all $k \geq 2$

$$
\int_{\Omega \cap B\left(\mathbf{x}_{0}, r\right)}\left|\nabla^{k} \mathbf{u}\right|^{2} d \mathbf{x} \leq \frac{c_{k}^{\prime}}{(R-r)^{2}} \int_{\Omega \cap B\left(\mathbf{x}_{0}, R\right)}\left|\nabla^{k-1} \mathbf{u}\right|^{2} d \mathbf{x} .
$$

We begin by proving this inequality for $k=2$. Let $\tau$ and $\nu$ be the tangent and normal vectors to $\Gamma$ respectively. By a standard difference quotient argument and Korn's inequality, we get that $\frac{\partial \mathbf{u}}{\partial \tau} \in W^{1,2}\left(\Omega \cap B\left(\mathbf{x}_{0}, R\right) ; \mathbb{R}^{2}\right)$ and

$$
\int_{\Omega} \mathbf{E}(\varphi) \cdot C\left[\mathbf{E}\left(\frac{\partial \mathbf{u}}{\partial \tau}\right)\right] d \mathbf{x}=0
$$

for all $\varphi \in W^{1,2}\left(\Omega ; \mathbb{R}^{2}\right)$ vanishing in a neighborhood of $\partial \Omega \backslash \Gamma$. Choosing now $\varphi:=\eta^{2} \frac{\partial \mathbf{u}}{\partial \tau}$, where $\eta$ is a smooth cut-off function such that $\eta=1$ in $B\left(\mathbf{x}_{0}, r\right)$ and $\eta=0$ outside $B\left(\mathbf{x}_{0}, R\right)$ and

$$
\|\nabla \eta\|_{\infty} \leq \frac{c}{R-r}
$$

we easily obtain

$$
\int_{\Omega} \eta^{2}\left|\mathbf{E}\left(\frac{\partial \mathbf{u}}{\partial \tau}\right)\right|^{2} d \mathbf{x} \leq c \int_{\Omega}|\nabla \eta|^{2}|\nabla \mathbf{u}|^{2} d \mathbf{x}
$$

Using Korn inequality once more gives

$$
\begin{aligned}
\int_{\Omega \cap B\left(\mathbf{x}_{0}, r\right)}\left|\nabla\left(\frac{\partial \mathbf{u}}{\partial \tau}\right)\right|^{2} d \mathbf{x} & \leq \int_{\Omega}\left|\nabla\left(\eta \frac{\partial \mathbf{u}}{\partial \tau}\right)\right|^{2} d \mathbf{x} \\
& \leq c \int_{\Omega}\left|\mathbf{E}\left(\eta \frac{\partial \mathbf{u}}{\partial \tau}\right)\right|^{2} d \mathbf{x} \leq c \int_{\Omega}|\nabla \eta|^{2}|\nabla \mathbf{u}|^{2} d \mathbf{x} \\
& \leq \frac{c}{(R-r)^{2}} \int_{\Omega \cap B\left(\mathbf{x}_{0}, R\right)}|\nabla \mathbf{u}|^{2} d \mathbf{x} .
\end{aligned}
$$

This provides $L^{2}$ estimates for $\frac{\partial^{2} \mathbf{u}}{\partial \tau^{2}}$ and $\frac{\partial^{2} \mathbf{u}}{\partial \tau \partial \nu}$. We now use the Lamé system to estimate $\frac{\partial^{2} \mathbf{u}}{\partial \nu^{2}}$ by rewriting it in terms of $\frac{\partial^{2} \mathbf{u}}{\partial \tau^{2}}$ and $\frac{\partial^{2} \mathbf{u}}{\partial \tau \partial \nu}$. Set

$$
(1,0)=\alpha \tau+\beta \nu, \quad(0,1)=\beta \tau-\alpha \nu,
$$


where $\alpha^{2}+\beta^{2}=1$. Since

$$
\frac{\partial \mathbf{u}}{\partial x}=\alpha \frac{\partial \mathbf{u}}{\partial \tau}+\beta \frac{\partial \mathbf{u}}{\partial \nu}, \quad \frac{\partial \mathbf{u}}{\partial y}=\beta \frac{\partial \mathbf{u}}{\partial \tau}-\alpha \frac{\partial \mathbf{u}}{\partial \nu}
$$

the Lamé system now becomes

$$
\begin{aligned}
\frac{\partial^{2} u_{1}}{\partial \nu^{2}}\left[\mu+(\mu+\lambda) \beta^{2}\right]-\frac{\partial^{2} u_{2}}{\partial \nu^{2}}(\mu+\lambda) \alpha \beta & =f_{1}, \\
-\frac{\partial^{2} u_{1}}{\partial \nu^{2}}(\mu+\lambda) \alpha \beta+\frac{\partial^{2} u_{2}}{\partial \nu^{2}}\left[\mu+(\mu+\lambda) \alpha^{2}\right] & =f_{2},
\end{aligned}
$$

where $f_{1}$ and $f_{2}$ are linear combinations of the remaining second order derivatives of $u_{1}$ and $u_{2}$ with coefficients depending only on the Lamé moduli and quadratic expressions of $\alpha$ and $\beta$. Hence

$$
\begin{aligned}
\frac{\partial^{2} u_{1}}{\partial \nu^{2}} & =\frac{f_{1}\left[\mu+(\mu+\lambda) \alpha^{2}\right]+f_{2}(\mu+\lambda) \alpha \beta}{(2 \mu+\lambda) \mu}, \\
\frac{\partial^{2} u_{2}}{\partial \nu^{2}} & =\frac{f_{2}\left[\mu+(\mu+\lambda) \beta^{2}\right]+f_{1}(\mu+\lambda) \alpha \beta}{(2 \mu+\lambda) \mu},
\end{aligned}
$$

which by (3.48) proves (3.47) with $k=2$. Since $\frac{\partial \mathbf{u}}{\partial \tau}$ satisfies (3.45) and (3.46) we can now repeat the same argument to get (3.47) with $k>2$.

Next we prove that for a local minimizer $(\mathbf{u}, \Omega)$ the domain $\Omega$ cannot have corners, i.e. at every point $\mathbf{z}_{0} \in \Gamma \backslash\left(\Gamma_{\text {cusps }} \cup \Gamma_{\text {cuts }}\right)$ the left and right derivatives of the Lipschitz function $g$ given in Proposition 3.5(i) must coincide. We use a blow-up argument which relies on the following result (see Theorem 3.1 and Remarks 1.1 and 5.1 in [18]).

Let $\Omega$ be a bounded open set in $\mathbb{R}^{2}$ whose boundary can be decomposed in three curves

$$
\partial \Omega=\Gamma_{1} \cup \Gamma_{2} \cup \Gamma_{3},
$$

where $\Gamma_{1}$ and $\Gamma_{2}$ are two segments meeting at the origin with an (internal) angle $\omega \in(0,2 \pi)$ and $\Gamma_{3}$ is a regular curve joining the two remaining endpoints of $\Gamma_{1}$ and $\Gamma_{2}$ in a smooth way. Denote by $\omega_{0} \in(\pi, 2 \pi)$ the solution of the equation

$$
\omega_{0}=\tan \omega_{0}
$$

Theorem 3.8 Let $\Omega \subset \mathbb{R}^{2}$ be as above and let $\mathbf{w} \in W^{1,2}\left(\Omega ; \mathbb{R}^{2}\right)$ be a weak solution of the Neumann problem

$$
\begin{aligned}
\mu \Delta \mathbf{w}+(\lambda+\mu) \nabla(\operatorname{div} \mathbf{w}) & =\mathbf{f}+\mu \mathbf{w} \quad \text { in } \Omega, \\
{\left[\mu\left(\nabla \mathbf{w}+\nabla \mathbf{w}^{T}\right)+\lambda(\operatorname{div} \mathbf{w}) I\right] \nu } & =\mathbf{0} \quad \text { on } \partial \Omega,
\end{aligned}
$$

where $\mathbf{f} \in L^{2}\left(\Omega ; \mathbb{R}^{2}\right)$. Then

(i) if $\omega \in(0, \pi]$ then $\mathbf{w} \in W^{2,2}\left(\Omega ; \mathbb{R}^{2}\right)$ and

$$
\|\mathbf{w}\|_{W^{2,2}\left(\Omega ; \mathbb{R}^{2}\right)} \leq c(\Omega)\|\mathbf{f}\|_{L^{2}\left(\Omega ; \mathbb{R}^{2}\right)} ;
$$

(ii) if $\omega \in(\pi, 2 \pi), \omega \neq \omega_{0}$, then $\mathbf{w}$ may be decomposed as

$$
\mathbf{w}=\mathbf{w}_{\mathrm{reg}}+\sum_{\alpha} c_{\alpha} \mathcal{S}_{\alpha}
$$

where $\alpha$ ranges among all complex numbers with $\operatorname{Re} \alpha \in(0,1)$ which are solutions of the equation

$$
\sin ^{2} \alpha \omega=\alpha^{2} \sin ^{2} \omega
$$


the functions $\mathcal{S}_{\alpha}$ are independent of $\mathbf{f}$ and in polar coordinates

$$
\mathcal{S}_{\alpha}(r, \theta)=r^{\alpha} \mathbf{g}_{\alpha}(r, \theta),
$$

with $\mathbf{g}_{\alpha} \in W^{2,2}\left(\Omega ; \mathbb{R}^{2}\right)$. Moreover

$$
\left\|\mathbf{w}_{\text {reg }}\right\|_{W^{2,2}\left(\Omega ; \mathbb{R}^{2}\right)}+\sum_{\alpha}\left|c_{\alpha}\right| \leq c(\Omega)\|\mathbf{f}\|_{L^{2}\left(\Omega ; \mathbb{R}^{2}\right)}
$$

(iii) if $\omega=\omega_{0}$ then $\mathbf{w}$ may be decomposed as in (3.49) with the only difference that $\alpha$ ranges among all complex numbers with $\operatorname{Re} \alpha \in(0,1]$ which are solutions of $(3.50)$ and the estimate (3.51) should be replaced by

$$
\left\|\mathbf{w}_{\text {reg }}\right\|_{W^{s, 2}\left(\Omega ; \mathbb{R}^{2}\right)}+\sum_{\alpha}\left|c_{\alpha}\right| \leq c(s, \Omega)\|\mathbf{f}\|_{L^{2}\left(\Omega ; \mathbb{R}^{2}\right)}
$$

for every $1<s<2$.

In addition it was shown in Theorem 2.2 in [25] that the following holds

Theorem 3.9 If $\omega \in(0,2 \pi)$ then the equation (3.50) has no solutions with $\operatorname{Re} \alpha \in\left(0, \frac{1}{2}\right]$.

From the previous two theorems we derive the following Decay Estimate for solutions of the Lamé system at a corner point.

Theorem 3.10 (Decay Estimate I) Let $\Omega$ be as in Theorem 3.8 with $\Gamma_{3}$ piecewise smooth and $r_{0}$ such that $\bar{B}\left(\mathbf{0}, r_{0}\right) \cap \Gamma_{3}=\emptyset$. Then there exist a constant $c>0$ and an exponent $\beta \in\left(\frac{1}{2}, 1\right)$ such that for every weak solution $\mathbf{u} \in W^{1,2}\left(\Omega ; \mathbb{R}^{2}\right)$ of the Lamé problem

$$
\begin{aligned}
\mu \Delta \mathbf{u}+(\lambda+\mu) \nabla(\operatorname{div} \mathbf{u})=\mathbf{0} & \text { in } \Omega, \\
{\left[\mu\left(\nabla \mathbf{u}+\nabla \mathbf{u}^{T}\right)+\lambda(\operatorname{div} \mathbf{u}) I\right] \nu } & =\mathbf{0} \quad \text { on } \Gamma_{1} \cup \Gamma_{2},
\end{aligned}
$$

we have the following decay estimate

$$
\int_{B(\mathbf{0}, r) \cap \Omega}|\nabla \mathbf{u}|^{2} d \mathbf{x} \leq c r^{2 \beta} \int_{\Omega}\left(|\mathbf{u}|^{2}+|\nabla \mathbf{u}|^{2}\right) d \mathbf{x}
$$

for all $0<r<r_{0}$.

Before proving the theorem we need the following auxiliary lemma.

Lemma 3.11 Let $\Omega^{\prime} \subset \mathbb{R}^{2}$ be as in Theorem 3.8 and let $\mathbf{g} \in W^{\frac{1}{2}, 2}\left(\partial \Omega^{\prime} ; \mathbb{R}^{2}\right)$ be a function vanishing in a neighborhood of the origin. Then there exist a function $\mathbf{v} \in W^{2,2}\left(\Omega^{\prime} ; \mathbb{R}^{2}\right)$ such that

$$
\left[\mu\left(\nabla \mathbf{v}+\nabla \mathbf{v}^{T}\right)+\lambda(\operatorname{div} \mathbf{v}) I\right] \nu=\mathbf{g} \quad \text { on } \partial \Omega^{\prime}
$$

and

$$
\|\mathbf{v}\|_{W^{2,2}\left(\Omega^{\prime} ; \mathbb{R}^{2}\right)} \leq c\left(\Omega^{\prime}\right)\|\mathbf{g}\|_{W^{\frac{1}{2}, 2}\left(\partial \Omega^{\prime} ; \mathbb{R}^{2}\right)}
$$

Proof. Writing $\mathbf{v}=\left(v_{1}, v_{2}\right), \nu=\left(\nu_{1}, \nu_{2}\right)$ and $\mathbf{g}=\left(g_{1}, g_{2}\right)$, a straightforward calculation shows that the equality

$$
\left[\mu\left(\nabla \mathbf{v}+\nabla \mathbf{v}^{T}\right)+\lambda(\operatorname{div} \mathbf{v}) I\right] \nu=\mathbf{g}
$$


is equivalent to

$$
\begin{aligned}
& \frac{\partial v_{1}}{\partial x}(2 \mu+\lambda) \nu_{1}+\frac{\partial v_{1}}{\partial y} \mu \nu_{2}+\frac{\partial v_{2}}{\partial x} \mu \nu_{2}+\frac{\partial v_{2}}{\partial y} \lambda \nu_{1}=g_{1} \\
& \frac{\partial v_{1}}{\partial x} \lambda \nu_{2}+\frac{\partial v_{1}}{\partial y} \mu \nu_{1}+\frac{\partial v_{2}}{\partial x} \mu \nu_{1}+\frac{\partial v_{2}}{\partial y}(2 \mu+\lambda) \nu_{2}=g_{2}
\end{aligned}
$$

Since

$$
\frac{\partial v}{\partial x}=\frac{\partial v}{\partial \nu} \nu_{1}+\frac{\partial v}{\partial \tau} \nu_{2}, \quad \frac{\partial v}{\partial y}=\frac{\partial v}{\partial \nu} \nu_{2}-\frac{\partial v}{\partial \tau} \nu_{1}
$$

the previous equation can be rewritten as

$$
\begin{aligned}
& \frac{\partial v_{1}}{\partial \nu}\left[\mu+(\mu+\lambda) \nu_{1}^{2}\right]+\frac{\partial v_{1}}{\partial \tau}(\mu+\lambda) \nu_{1} \nu_{2}+\frac{\partial v_{2}}{\partial \nu}(\mu+\lambda) \nu_{1} \nu_{2}+\frac{\partial v_{2}}{\partial \tau}\left(\mu \nu_{2}^{2}-\lambda \nu_{1}^{2}\right)=g_{1} \\
& \frac{\partial v_{1}}{\partial \nu}(\mu+\lambda) \nu_{1} \nu_{2}+\frac{\partial v_{1}}{\partial \tau}\left(\lambda \nu_{2}^{2}-\mu \nu_{1}^{2}\right)+\frac{\partial v_{2}}{\partial \nu}\left[\mu+(\mu+\lambda) \nu_{2}^{2}\right]-\frac{\partial v_{2}}{\partial \tau}(\mu+\lambda) \nu_{1} \nu_{2}=g_{2} .
\end{aligned}
$$

Choosing $\frac{\partial v_{1}}{\partial \tau}=\frac{\partial v_{2}}{\partial \tau}=0$ the previous system becomes

$$
\begin{aligned}
& \frac{\partial v_{1}}{\partial \nu}\left[\mu+(\mu+\lambda) \nu_{1}^{2}\right]+\frac{\partial v_{2}}{\partial \nu}(\mu+\lambda) \nu_{1} \nu_{2}=g_{1} \\
& \frac{\partial v_{1}}{\partial \nu}(\mu+\lambda) \nu_{1} \nu_{2}+\frac{\partial v_{2}}{\partial \nu}\left[\mu+(\mu+\lambda) \nu_{2}^{2}\right]=g_{2}
\end{aligned}
$$

which yields

$$
\begin{aligned}
\frac{\partial v_{1}}{\partial \nu} & =\frac{g_{1}\left[\mu+(\mu+\lambda) \nu_{2}^{2}\right]-g_{2}(\mu+\lambda) \nu_{1} \nu_{2}}{(2 \mu+\lambda) \mu}, \\
\frac{\partial v_{2}}{\partial \nu} & =\frac{g_{2}\left[\mu+(\mu+\lambda) \nu_{1}^{2}\right]-g_{1}(\mu+\lambda) \nu_{1} \nu_{2}}{(2 \mu+\lambda) \mu} .
\end{aligned}
$$

Note that even if $\nu$ is discontinuous at the origin by the assumption on $\mathbf{g}$ the right hand sides of the previous equations are zero in a neighborhood of the origin, hence are both in the space $W^{\frac{1}{2}, 2}\left(\partial \Omega^{\prime}\right)$. We can now apply Theorem 1.5.2.8 of [17] to get a function $\mathbf{v} \in W^{2,2}\left(\Omega^{\prime} ; \mathbb{R}^{2}\right)$ such that its trace $\mathbf{v}=\mathbf{0}$ on $\partial \Omega^{\prime}$, the components of its normal derivative satisfy (3.53) and (3.54), and its $W^{2,2}$ norm is bounded by the $W^{\frac{1}{2}, 2}$ norm of the right hand side of (3.53) and (3.54)

$$
\|\mathbf{v}\|_{W^{2,2}\left(\Omega^{\prime} ; \mathbb{R}^{2}\right)} \leq c\left(\Omega^{\prime}\right)\|\mathbf{g}\|_{W^{\frac{1}{2}, 2}\left(\partial \Omega^{\prime} ; \mathbb{R}^{2}\right)} .
$$

which concludes the proof.

We are now ready to prove the theorem.

Proof. Let $\omega$ be the angle of $\Omega$ at the origin. We only give the proof in the case where $\omega$ satisfies condition (ii) of Theorem 3.8, that is $\omega \in(\pi, 2 \pi), \omega \neq \omega_{0}$, since case (i) is significantly simpler, while case (iii) is completely analogous to case (ii).

Let $\Omega^{\prime} \subset \Omega$ be as in Theorem 3.8 and such that $\Omega^{\prime} \supset \Omega \cap B\left(\mathbf{0}, r_{0}\right)$ and the distance between $\Gamma_{3}^{\prime}$ and $\Gamma_{3}$ is strictly positive, where $\Gamma_{3}^{\prime}:=\Omega \cap \partial \Omega^{\prime}$. By Theorem 3.7 we have that $\mathbf{u}$ is $C^{\infty}$ outside a neighborhood $U$ of the origin. Moreover

$$
\int_{\Omega^{\prime} \backslash U}\left|\nabla^{2} \mathbf{u}\right|^{2} d \mathbf{x} \leq c \int_{\Omega}|\nabla \mathbf{u}|^{2} d \mathbf{x} .
$$

Set

$$
\sigma(\mathbf{u}):=\left[\mu\left(\nabla \mathbf{u}+\nabla \mathbf{u}^{T}\right)+\lambda(\operatorname{div} \mathbf{u}) I\right]
$$


Since $\sigma(\mathbf{u}) \nu=0$ on $U \cap \partial \Omega^{\prime}$ and is smooth we are in position to apply the previous lemma with $\mathbf{g}=\sigma(\mathbf{u}) \nu$ to find a function $\mathbf{v} \in W^{2,2}\left(\Omega^{\prime} ; \mathbb{R}^{2}\right)$ such that

$$
\left[\mu\left(\nabla \mathbf{v}+\nabla \mathbf{v}^{T}\right)+\lambda(\operatorname{div} \mathbf{v}) I\right] \nu=\sigma(\mathbf{u}) \nu \quad \text { on } \partial \Omega^{\prime}
$$

and

$$
\begin{aligned}
\|\mathbf{v}\|_{W^{2,2}\left(\Omega^{\prime} ; \mathbb{R}^{2}\right)} & \leq c\left(\Omega^{\prime}\right)\|\sigma(\mathbf{u}) \nu\|_{W^{\frac{1}{2}, 2}\left(\partial \Omega^{\prime} ; \mathbb{R}^{2}\right)} \\
& \leq c\left(\Omega^{\prime}\right)\left(\int_{\Omega^{\prime} \backslash U}\left(|\nabla \mathbf{u}|^{2}+\left|\nabla^{2} \mathbf{u}\right|^{2}\right) d \mathbf{x}\right)^{\frac{1}{2}} .
\end{aligned}
$$

Therefore from the estimate (3.55) we conclude that

$$
\|\mathbf{v}\|_{W^{2,2}\left(\Omega^{\prime} ; \mathbb{R}^{2}\right)} \leq c(\Omega)\|\nabla \mathbf{u}\|_{L^{2}\left(\Omega ; \mathbb{R}^{2 \times 2}\right)} .
$$

Defining $\mathbf{w}:=\mathbf{u}-\mathbf{v}$ we get that $\mathbf{w}$ is a weak solution of

$$
\begin{aligned}
\mu \Delta \mathbf{w}+(\lambda+\mu) \nabla(\operatorname{div} \mathbf{w}) & =\mathbf{f}+\mu \mathbf{w} \quad \text { in } \Omega^{\prime} \\
{\left[\mu\left(\nabla \mathbf{w}+\nabla \mathbf{w}^{T}\right)+\lambda(\operatorname{div} \mathbf{w}) I\right] \nu } & =\mathbf{0} \quad \text { on } \partial \Omega^{\prime}
\end{aligned}
$$

where

$$
\mathbf{f}:=\mu \Delta \mathbf{v}+(\lambda+\mu) \nabla(\operatorname{div} \mathbf{v})-\mu(\mathbf{u}-\mathbf{v})
$$

By (3.56) we have that

$$
\|\mathbf{f}\|_{L^{2}\left(\Omega^{\prime} ; \mathbb{R}^{2}\right)} \leq c(\Omega)\|\mathbf{u}\|_{W^{1,2}\left(\Omega ; \mathbb{R}^{2}\right)} .
$$

By Theorem 3.8 we may write

$$
\mathbf{w}=\mathbf{w}_{\text {reg }}+\sum_{\alpha} c_{\alpha} \mathcal{S}_{\alpha}
$$

so that

$$
\left\|\mathbf{w}_{\mathrm{reg}}\right\|_{W^{2,2}\left(\Omega^{\prime} ; \mathbb{R}^{2}\right)}+\sum_{\alpha}\left|c_{\alpha}\right| \leq c\left(\Omega^{\prime}\right)\|\mathbf{f}\|_{L^{2}\left(\Omega ; \mathbb{R}^{2}\right)} \leq c(\Omega)\|\mathbf{u}\|_{W^{1,2}\left(\Omega ; \mathbb{R}^{2}\right)} .
$$

Here and in the remaining part of the proof $\alpha$ ranges among all complex numbers with $\operatorname{Re} \alpha \in(0,1)$ which are solutions of the equation (3.50). Using Sobolev Embedding Theorem and the previous estimate we have for any $p>2$ and $0<r \leq r_{0}$

$$
\begin{aligned}
& \int_{B(\mathbf{0}, r) \cap \Omega}|\nabla \mathbf{u}|^{2} d \mathbf{x} \leq 2\left(\int_{B(\mathbf{0}, r) \cap \Omega}|\nabla \mathbf{w}|^{2} d \mathbf{x}+\int_{B(\mathbf{0}, r) \cap \Omega}|\nabla \mathbf{v}|^{2} d \mathbf{x}\right) \\
& \leq c\left(\int_{B(\mathbf{0}, r) \cap \Omega}\left|\nabla \mathbf{w}_{\mathrm{reg}}\right|^{2} d \mathbf{x}+\int_{B(\mathbf{0}, r) \cap \Omega}|\nabla \mathbf{v}|^{2} d \mathbf{x}\right)+c \sum_{\alpha}\left|c_{\alpha}\right|^{2} \int_{B(\mathbf{0}, r) \cap \Omega}\left|\nabla \mathcal{S}_{\alpha}\right|^{2} d \mathbf{x} \\
& \leq c\left(\int_{B(\mathbf{0}, r) \cap \Omega}\left|\nabla \mathbf{w}_{\mathrm{reg}}\right|^{p} d \mathbf{x}+\int_{B(\mathbf{0}, r) \cap \Omega}|\nabla \mathbf{v}|^{p} d \mathbf{x}\right)^{\frac{2}{p}} r^{2-\frac{4}{p}}+c \sum_{\alpha}\left|c_{\alpha}\right|^{2} r^{2 \operatorname{Re} \alpha} \\
& \leq c\left(\left\|\mathbf{w}_{\mathrm{reg}}\right\|_{W^{2,2}\left(\Omega^{\prime} ; \mathbb{R}^{2}\right)}^{2}+\|\mathbf{v}\|_{W^{2,2}\left(\Omega^{\prime} ; \mathbb{R}^{2}\right)}^{2}\right) r^{2-\frac{4}{p}}+c\|\mathbf{f}\|_{L^{2}\left(\Omega^{\prime} ; \mathbb{R}^{2}\right)}^{2} \sum_{\alpha} r^{2 \operatorname{Re} \alpha} \\
& \leq c\|\mathbf{u}\|_{W^{1,2}\left(\Omega ; \mathbb{R}^{2}\right)}^{2}\left(r^{2-\frac{4}{p}}+\sum_{\alpha} r^{2 \operatorname{Re} \alpha}\right) .
\end{aligned}
$$

Choosing $p$ so large that

$$
1-\frac{2}{p} \geq \beta:=\min _{\alpha}\{\operatorname{Re} \alpha\}
$$


and recalling Theorem 3.9, we obtain (3.52) for all $0<r \leq r_{0}$.

We now use a blow-up argument to show that for a local minimizer $(\mathbf{u}, \Omega) \in X$ for the functional $F_{\infty}$ defined in (3.1) the domain $\Omega$ cannot have corners, i.e. that at every point $\mathbf{z}_{0} \in \Gamma \backslash\left(\Gamma_{\text {cusps }} \cup \Gamma_{\text {cuts }}\right)$ the left and right derivatives of the Lipschitz function $g$ given in Proposition 3.5(i) must coincide.

Theorem 3.12 (Blow-Up) Let $(\mathbf{u}, \Omega) \in X$ be a $\delta$-local minimizer for the functional $F_{\infty}$ defined in (3.1). Assume that $\Gamma$ has a corner at some point $\mathbf{z}_{0} \in \Gamma \backslash\left(\Gamma_{\text {cusps }} \cup \Gamma_{\text {cuts }}\right)$. Then there exists a constant $c>0$, a radius $r_{0}$, and an exponent $\frac{1}{2}<\alpha<1$ such that

$$
\int_{B\left(\mathbf{z}_{0}, r\right) \cap \Omega}|\nabla \mathbf{u}|^{2} d \mathbf{x} \leq c r^{2 \alpha}
$$

for all $0<r<r_{0}$

Proof. Step 1: We claim that there exist an orthonormal basis $\left\{\mathbf{e}_{1}, \mathbf{e}_{2}\right\}$ of $\mathbb{R}^{2}$, three constants $c_{1}, L>0, \tau_{0} \in(0,1)$, and an exponent $\frac{1}{2}<\beta<1$ such that for all $\tau \in\left(0, \tau_{0}\right)$ there exists a radius $0<r_{\tau}<1$ such that

$$
\int_{C\left(\mathbf{z}_{0}, \tau r\right)}|\nabla \mathbf{u}|^{2} d \mathbf{x} \leq c_{1} \tau^{2 \beta} \int_{C\left(\mathbf{z}_{0}, r\right)}\left(1+|\nabla \mathbf{u}|^{2}\right) d \mathbf{x}
$$

for all $0<r<r_{\tau}$, where

$$
C\left(\mathbf{z}_{0}, r\right):=\Omega \cap\left\{\mathbf{z}_{0}+s \mathbf{e}_{1}+t \mathbf{e}_{2}:-r<s<r,-4 L r<t<4 L r\right\} .
$$

By Proposition 3.5(i) there exists a Lipschitz function $g:\left(-a^{\prime}, a^{\prime}\right) \rightarrow\left(-b^{\prime}, b^{\prime}\right)$, with $\operatorname{Lip} g \leq L$ for some $L>1$, such that $g(0)=0$ and

$$
\Omega \cap Q=\left\{\mathbf{z}_{0}+s \mathbf{e}_{1}+t \mathbf{e}_{2}:-a^{\prime}<s<a^{\prime},-b^{\prime}<t<g(s)\right\},
$$

for some $a^{\prime}, b^{\prime}$, where

$$
Q:=\left\{\mathbf{z}_{0}+s \mathbf{e}_{1}+t \mathbf{e}_{2}:-a^{\prime}<s<a^{\prime},-b^{\prime}<t<b^{\prime}\right\} .
$$

Moreover the function $g$ admits left and right derivatives at every point, that are respectively left and right continuous. Since $\Gamma$ has a corner at $\mathbf{z}_{0}$ we have $g_{-}^{\prime}(0) \neq g_{+}^{\prime}(0)$. By Korn's Inequality in Lipschitz domains we may assume that $\mathbf{u} \in H^{1}\left(\Omega \cap Q ; \mathbb{R}^{2}\right)$.

Note that for all $0<r \leq \min \left\{a^{\prime}, \frac{b^{\prime}}{4 L}\right\}$

$$
C\left(\mathbf{z}_{0}, r\right)=\left\{\mathbf{z}_{0}+s \mathbf{e}_{1}+t \mathbf{e}_{2}:-r<s<r,-4 L r<t<g(s)\right\} .
$$

Fix $c_{1}>0, \tau_{0} \in(0,1)$ and $\beta>\frac{1}{2}$ to be determined later and assume by contradiction that the corresponding estimate $(3.58)$ is false for some $\tau \in\left(0, \tau_{0}\right)$. Hence we may find a sequence of radii $\left\{r_{n}\right\}$ converging to zero such that

$$
\int_{C\left(\mathbf{z}_{0}, \tau r_{n}\right)}|\nabla \mathbf{u}|^{2} d \mathbf{x}>c_{1} \tau^{2 \beta} \int_{C\left(\mathbf{z}_{0}, r_{n}\right)}\left(1+|\nabla \mathbf{u}|^{2}\right) d \mathbf{x}
$$

Define the sets

$$
\begin{aligned}
C_{n} & :=\frac{1}{r_{n}}\left(-\mathbf{z}_{0}+C\left(\mathbf{z}_{0}, r_{n}\right)\right) \\
& =\left\{s \mathbf{e}_{1}+t \mathbf{e}_{2}:-1<s<1,-4 L<t<\frac{g\left(r_{n} s\right)-g(0)}{r_{n}}\right\} .
\end{aligned}
$$


Then $\chi_{C_{n}}$ converges a.e. to the $\chi_{C_{\infty}}$ where

$$
C_{\infty}:=\left\{s \mathbf{e}_{1}+t \mathbf{e}_{2}:-1<s<1,-4 L<t<g_{\infty}(s)\right\}
$$

with $g_{\infty}(s):=g_{-}^{\prime}(0) s$ for $s<0$ and $g_{\infty}(s):=g_{+}^{\prime}(0) s$ for $s>0$. We rescale accordingly also the function $\mathbf{u}$ by setting

$$
\mathbf{u}_{n}(\mathbf{z}):=\frac{\mathbf{u}\left(\mathbf{z}_{0}+r_{n} \mathbf{z}\right)-\mathbf{a}_{n}}{\lambda_{n} r_{n}},
$$

where $\mathbf{z}:=\frac{\mathbf{x}-\mathbf{z}_{0}}{r_{n}}$,

$$
\mathbf{a}_{n}:=\frac{1}{\left|C\left(\mathbf{z}_{0}, r_{n}\right)\right|} \int_{C\left(\mathbf{z}_{0}, r_{n}\right)} \mathbf{u} d \mathbf{x}, \quad \lambda_{n}^{2}:=\frac{1}{\left|C\left(\mathbf{z}_{0}, r_{n}\right)\right|} \int_{C\left(\mathbf{z}_{0}, r_{n}\right)}|\nabla \mathbf{u}|^{2} d \mathbf{x} .
$$

Note that

$$
\frac{1}{\left|C_{n}\right|} \int_{C_{n}}\left|\nabla \mathbf{u}_{n}\right|^{2} d \mathbf{z}=1
$$

Moreover, since by construction $\int_{C_{n}} \mathbf{u}_{n} d \mathbf{z}=\mathbf{0}$, by Poincaré inequality and a standard extension argument we may extend each function $\mathbf{u}_{n}$ to the rectangle

$$
R:=\left\{s \mathbf{e}_{1}+t \mathbf{e}_{2}:-1<s<1,-4 L<t<4 L\right\},
$$

in such a way that the resulting function (still denoted $\left.\mathbf{u}_{n}\right)$ belongs to $W^{1,2}\left(R ; \mathbb{R}^{2}\right)$ and satisfies

$$
\left\|\mathbf{u}_{n}\right\|_{W^{1,2}\left(R ; \mathbb{R}^{2}\right)} \leq c(L)\left\|\nabla \mathbf{u}_{n}\right\|_{L^{2}\left(C_{n} ; \mathbb{R}^{2 \times 2}\right)} \leq c .
$$

Without loss of generality we may assume that the sequence $\left\{\mathbf{u}_{n}\right\}$ weakly converges to some function $\mathbf{u}_{\infty} \in W^{1,2}\left(R ; \mathbb{R}^{2}\right)$ and that

$$
\lambda_{n} \rightarrow \lambda_{\infty} \in[0, \infty] .
$$

Note that by (3.60) necessarily $\lambda_{\infty}>0$. Moreover, denoting by $x_{0}$ the point in $(a, b)$ such that $\mathbf{z}_{0}=\left(x_{0}, h\left(x_{0}\right)\right)$, it is easy to see that the functions $\mathbf{u}_{n}$ satisfy the equation

$$
\int_{C_{n}} \mathbf{E}(\varphi) \cdot C\left[\mathbf{E}\left(\mathbf{u}_{n}\right)\right] d \mathbf{z}=\frac{1}{\lambda_{n}} \int_{C_{n}} \mathbf{E}(\varphi) \cdot C\left[\mathbf{E}_{0}\left(h\left(x_{0}\right)+r_{n} z_{2}\right)\right] d \mathbf{z}
$$

for every $\varphi \in C_{0}^{1}\left(R ; \mathbb{R}^{2}\right)$. We observe that the sequence $\left\{\mathbf{E}_{0}\left(h\left(x_{0}\right)+r_{n} \cdot\right)\right\}$ converges to $\mathbf{E}_{\infty}$ in $L^{p}\left(R ; \mathbb{R}^{2 \times 2}\right)$ for any $1 \leq p<\infty$, where

$$
\mathbf{E}_{\infty}(\mathbf{z}):=e_{\infty}\left(z_{2}\right) \mathbf{i} \otimes \mathbf{i}, \quad e_{\infty}\left(z_{2}\right):= \begin{cases}e_{0} & \text { if } h\left(x_{0}\right)>0 \\ \chi_{\left\{z_{2}>0\right\}} e_{0} & \text { if } h\left(x_{0}\right)=0 .\end{cases}
$$

Step 2: We now fix a ball $B$ such that

$$
B \subset \subset\left\{s \mathbf{e}_{1}+t \mathbf{e}_{2}:-1<s<1,-4 L<t<-3 L\right\} .
$$

We claim that for all functions $\psi \in C_{0}^{1}(R)$ which vanish in $B$ we have

$$
\lim _{n \rightarrow \infty} \int_{C_{n}} \psi^{2}\left|\nabla \mathbf{u}_{n}-\nabla \mathbf{u}_{\infty}\right|^{2} d \mathbf{z}=0 .
$$

From (3.61), and the fact that $\chi_{C_{n}} \rightarrow \chi_{C_{\infty}}$ in $L^{2}(R), \mathbf{u}_{n} \rightarrow \mathbf{u}_{\infty}$ in $W^{1,2}\left(R ; \mathbb{R}^{2}\right)$, and

$$
\mathbf{E}_{0}\left(h\left(x_{0}\right)+r_{n} \cdot\right) \rightarrow \mathbf{E}_{\infty} \text { in } L^{p}\left(R ; \mathbb{R}^{2 \times 2}\right)
$$


we get that

$$
\int_{C_{\infty}} \mathbf{E}(\varphi) \cdot C\left[\mathbf{E}\left(\mathbf{u}_{\infty}\right)\right] d \mathbf{z}=\frac{1}{\lambda_{\infty}} \int_{C_{\infty}} \mathbf{E}(\varphi) \cdot C\left[\mathbf{E}_{\infty}\right] d \mathbf{z},
$$

where the right-hand side is understood to be zero when $\lambda_{\infty}=\infty$.

Fix $\psi \in C_{0}^{1}(R)$ and choose $\varphi:=\psi^{2} \mathbf{u}_{n}$ in (3.61) $\left(\varphi:=\psi^{2} \mathbf{u}_{\infty}\right.$ in (3.62) respectively) thus getting

$$
\begin{aligned}
\int_{C_{n}} \psi^{2} \mathbf{E}\left(\mathbf{u}_{n}\right) & \cdot C\left[\mathbf{E}\left(\mathbf{u}_{n}\right)\right] d \mathbf{z}=\frac{1}{\lambda_{n}} \int_{C_{n}} \mathbf{E}\left(\psi^{2} \mathbf{u}_{n}\right) \cdot C\left[\mathbf{E}_{0}\left(h\left(x_{0}\right)+r_{n} z_{2}\right)\right] d \mathbf{z} \\
& -\int_{C_{n}} \psi\left(\mathbf{u}_{n} \otimes \nabla \psi+\left(\mathbf{u}_{n} \otimes \nabla \psi\right)^{T}\right) \cdot C\left[\mathbf{E}\left(\mathbf{u}_{n}\right)\right] d \mathbf{z}
\end{aligned}
$$

and

$$
\begin{aligned}
\int_{C_{\infty}} \psi^{2} \mathbf{E}\left(\mathbf{u}_{\infty}\right) & \cdot C\left[\mathbf{E}\left(\mathbf{u}_{\infty}\right)\right] d \mathbf{z}=\frac{1}{\lambda_{\infty}} \int_{C_{\infty}} \mathbf{E}\left(\psi^{2} \mathbf{u}_{\infty}\right) \cdot C\left[\mathbf{E}_{\infty}\right] d \mathbf{z} \\
& -\int_{C_{\infty}} \psi\left(\mathbf{u}_{\infty} \otimes \nabla \psi+\left(\mathbf{u}_{\infty} \otimes \nabla \psi\right)^{T}\right) \cdot C\left[\mathbf{E}\left(\mathbf{u}_{\infty}\right)\right] d \mathbf{z} .
\end{aligned}
$$

Letting $n \rightarrow \infty$ in (3.63), and using the fact that the right-hand side converges to the right-hand side of (3.64), we get that

$$
\lim _{n \rightarrow \infty} \int_{C_{n}} \psi^{2} \mathbf{E}\left(\mathbf{u}_{n}\right) \cdot C\left[\mathbf{E}\left(\mathbf{u}_{n}\right)\right] d \mathbf{z}=\int_{C_{\infty}} \psi^{2} \mathbf{E}\left(\mathbf{u}_{\infty}\right) \cdot C\left[\mathbf{E}\left(\mathbf{u}_{\infty}\right)\right] d \mathbf{z},
$$

or, equivalently,

$$
\lim _{n \rightarrow \infty} \int_{C_{n}} \psi^{2}\left\{\mathbf{E}\left(\mathbf{u}_{n}\right) \cdot C\left[\mathbf{E}\left(\mathbf{u}_{n}\right)\right]-\mathbf{E}\left(\mathbf{u}_{\infty}\right) \cdot C\left[\mathbf{E}\left(\mathbf{u}_{\infty}\right)\right]\right\} d \mathbf{z}=0
$$

from which we easily get

$$
\lim _{n \rightarrow \infty} \int_{C_{n}} \mathbf{E}\left(\psi\left(\mathbf{u}_{n}-\mathbf{u}_{\infty}\right)\right) \cdot C\left[\mathbf{E}\left(\psi\left(\mathbf{u}_{n}-\mathbf{u}_{\infty}\right)\right)\right] d \mathbf{z}=0
$$

Hence the claim follows from Theorem 4.2.

Step 3: We now divide the proof according to the three cases $\lambda_{\infty}=\infty, \lambda_{\infty}<\infty$ and $h\left(x_{0}\right)>0$, $\lambda_{\infty}<\infty$ and $h\left(x_{0}\right)=0$. We begin by assuming that $\lambda_{\infty}=\infty$. In this case it follows from (3.62) that $\mathbf{u}_{\infty}$ is a weak solution of the problem

$$
\begin{aligned}
\mu \Delta \mathbf{u}_{\infty}+(\lambda+\mu) \nabla\left(\operatorname{div} \mathbf{u}_{\infty}\right)=\mathbf{0} & \text { in } C_{\infty} \\
{\left[\mu\left(\nabla \mathbf{u}_{\infty}+\nabla \mathbf{u}_{\infty}^{T}\right)+\lambda\left(\operatorname{div} \mathbf{u}_{\infty}\right) I\right] \nu=\mathbf{0} } & \text { on } \Gamma_{g_{\infty}} .
\end{aligned}
$$

By Theorem 3.10 there exist $c>0$ and $\beta \in\left(\frac{1}{2}, 1\right)$ such that for all $0<r<1$ we have

$$
\begin{aligned}
\int_{B(\mathbf{0}, r) \cap C_{\infty}}\left|\nabla \mathbf{u}_{\infty}\right|^{2} d \mathbf{z} & \leq c r^{2 \beta} \int_{C_{\infty}}\left(\left|\mathbf{u}_{\infty}\right|^{2}+\left|\nabla \mathbf{u}_{\infty}\right|^{2}\right) d \mathbf{z} \\
& \leq c r^{2 \beta} \int_{C_{\infty}}\left|\nabla \mathbf{u}_{\infty}\right|^{2} d \mathbf{z} \leq c r^{2 \beta}
\end{aligned}
$$

where we have used Poincaré inequality, which holds since $\int_{C_{\infty}} \mathbf{u}_{\infty} d \mathbf{z}=0$, and the fact that $\int_{C_{\infty}}\left|\nabla \mathbf{u}_{\infty}\right|^{2} d \mathbf{z} \leq\left|C_{\infty}\right|$. Therefore if $\tau_{0}$ is such that

$$
\tau_{0} C_{\infty} \subset\left(B(\mathbf{0}, 1) \cap C_{\infty}\right) \backslash\left\{s \mathbf{e}_{1}+t \mathbf{e}_{2}:-1<s<1,-4 L<t<-3 L\right\}
$$


we get that for all $0<\tau \leq \tau_{0}$

$$
\int_{\tau C_{\infty}}\left|\nabla \mathbf{u}_{\infty}\right|^{2} d \mathbf{z} \leq \int_{B\left(\mathbf{0}, \frac{\tau}{\tau_{0}}\right) \cap C_{\infty}}\left|\nabla \mathbf{u}_{\infty}\right|^{2} d \mathbf{z} \leq c_{2} \tau^{2 \beta} .
$$

By Step 2 we then have that

$$
\lim _{n \rightarrow \infty} \int_{\tau C_{n}}\left|\nabla \mathbf{u}_{n}\right|^{2} d \mathbf{z}=\int_{\tau C_{\infty}}\left|\nabla \mathbf{u}_{\infty}\right|^{2} d \mathbf{z}
$$

for all $0<\tau \leq \tau_{0}$, and so

$$
\lim _{n \rightarrow \infty} \frac{\int_{C\left(\mathbf{z}_{0}, \tau r_{n}\right)}|\nabla \mathbf{u}|^{2} d \mathbf{x}}{\int_{C\left(\mathbf{z}_{0}, r_{n}\right)}|\nabla \mathbf{u}|^{2} d \mathbf{x}}=\frac{1}{\left|C_{\infty}\right|} \lim _{n \rightarrow \infty} \int_{\tau C_{n}}\left|\nabla \mathbf{u}_{n}\right|^{2} d \mathbf{z} \leq \frac{c_{2}}{\left|C_{\infty}\right|} \tau^{2 \beta}
$$

which contradicts (3.60), provided we take

$$
c_{1} \geq 2 \frac{c_{2}}{\left|C_{\infty}\right|} .
$$

Step 4: Assume next that $\lambda_{\infty}<\infty$ and $h\left(x_{0}\right)>0$. In this case $e_{\infty} \equiv e_{0}$. Define

$$
\mathbf{v}_{\infty}(\mathbf{z}):=\mathbf{u}_{\infty}(\mathbf{z})-\frac{\left(e_{0} z_{1}, 0\right)}{\lambda_{\infty}} .
$$

Then $\mathbf{v}_{\infty}$ is a weak solution of the problem

$$
\begin{aligned}
\mu \Delta \mathbf{v}_{\infty}+(\lambda+\mu) \nabla\left(\operatorname{div} \mathbf{v}_{\infty}\right)=\mathbf{0} & \text { in } C_{\infty} \\
{\left[\mu\left(\nabla \mathbf{v}_{\infty}+\nabla \mathbf{v}_{\infty}^{T}\right)+\lambda\left(\operatorname{div} \mathbf{v}_{\infty}\right) I\right] \nu=\mathbf{0} } & \text { on } \Gamma_{h_{\infty}}
\end{aligned}
$$

and thus, as in the previous step, for all $0<r<1$ we have

$$
\int_{B(\mathbf{0}, r) \cap C_{\infty}}\left|\nabla \mathbf{v}_{\infty}\right|^{2} d \mathbf{z} \leq c r^{2 \beta} \int_{C_{\infty}}\left(\left|\mathbf{v}_{\infty}\right|^{2}+\left|\nabla \mathbf{v}_{\infty}\right|^{2}\right) d \mathbf{z}
$$

from which we easily obtain that for all $0<\tau \leq \tau_{0}$, where $\tau_{0}$ is the same as in the previous step, there holds

$$
\int_{\tau C_{\infty}}\left|\nabla \mathbf{u}_{\infty}\right|^{2} d \mathbf{z} \leq c \tau^{2 \beta} \int_{C_{\infty}}\left(\left|\nabla \mathbf{u}_{\infty}\right|^{2}+\frac{1}{\lambda_{\infty}^{2}}\right) d \mathbf{z} \leq c_{3} \tau^{2 \beta}\left(1+\frac{1}{\lambda_{\infty}^{2}}\right) .
$$

In turn

$$
\begin{aligned}
\lim _{n \rightarrow \infty} \frac{\int_{C\left(\mathbf{z}_{0}, \tau r_{n}\right)}|\nabla \mathbf{u}|^{2} d \mathbf{x}}{\int_{C\left(\mathbf{z}_{0}, r_{n}\right)}\left(|\nabla \mathbf{u}|^{2}+1\right) d \mathbf{x}} & =\lim _{n \rightarrow \infty} \frac{\int_{C\left(\mathbf{z}_{0}, \tau r_{n}\right)}|\nabla \mathbf{u}|^{2} d \mathbf{x}}{\left|C_{\infty}\right|\left(\lambda_{n}^{2} r_{n}^{2}+r_{n}^{2}\right)} \\
& =\lim _{n \rightarrow \infty} \frac{\int_{\tau C_{n}}\left|\nabla \mathbf{u}_{n}\right|^{2} d \mathbf{z}}{\left|C_{\infty}\right|\left(1+\frac{1}{\lambda_{n}^{2}}\right)} \leq \frac{c_{3}}{\left|C_{\infty}\right|} \tau^{2 \beta}
\end{aligned}
$$

which is again a contradiction provided we take

$$
c_{1} \geq 2 \frac{c_{3}}{\left|C_{\infty}\right|} .
$$

Step 5: Finally we consider the case $\lambda_{\infty}<\infty$ and $h\left(x_{0}\right)=0$. Define

$$
\widetilde{e}_{\infty}\left(z_{2}\right):= \begin{cases}e_{0} & \text { if } z_{2}>0, \\ \gamma & \text { if } z_{2}<0,\end{cases}
$$


for some $\gamma$ to be determined later, and observe that for every $\varphi \in C_{0}^{1}\left(R ; \mathbb{R}^{2}\right)$

$$
\begin{aligned}
\int_{C_{\infty}} e_{\infty}\left(z_{2}\right) \frac{\partial \varphi_{1}}{\partial z_{1}} d \mathbf{z} & =\int_{C_{\infty} \cap\left\{z_{2}>0\right\}} e_{0} \frac{\partial \varphi_{1}}{\partial z_{1}} d \mathbf{z}=\int_{C_{\infty} \cap\left\{z_{2}>0\right\}} e_{0} \frac{\partial \varphi_{1}}{\partial z_{1}} d \mathbf{z} \\
+\int_{C_{\infty} \cap\left\{z_{2}<0\right\}} \gamma \frac{\partial \varphi_{1}}{\partial z_{1}} d \mathbf{z} & =\int_{C_{\infty}} \widetilde{e}_{\infty}\left(z_{2}\right) \frac{\partial \varphi_{1}}{\partial z_{1}} d \mathbf{z},
\end{aligned}
$$

where we have used the fact that

$$
\int_{C_{\infty} \cap\left\{z_{2}<0\right\}} \frac{\partial \varphi_{1}}{\partial z_{1}} d \mathbf{z}=\int_{C_{\infty} \cap\left\{z_{2}=0\right\}} \varphi_{1} \nu_{1} d \mathcal{H}^{1}\left(z_{1}, z_{2}\right)=0
$$

since $\nu_{1}=0$. Define

$$
\mathbf{w}_{\infty}(\mathbf{z}):=e_{0}\left(z_{1},-\min \left\{0, \frac{\lambda z_{2}}{2 \mu+\lambda}\right\}\right), \quad \gamma:=\frac{4 \mu(\mu+\lambda)}{(2 \mu+\lambda)^{2}} e_{0} .
$$

A straightforward calculation shows that

$$
\begin{aligned}
(2 \mu+\lambda) \widetilde{e}_{\infty} & =(2 \mu+\lambda) \frac{\partial\left(\mathbf{w}_{\infty}\right)_{1}}{\partial z_{1}}+\lambda \frac{\partial\left(\mathbf{w}_{\infty}\right)_{2}}{\partial z_{2}} \\
\lambda e_{\infty} & =\lambda \frac{\partial\left(\mathbf{w}_{\infty}\right)_{1}}{\partial z_{1}}+(2 \mu+\lambda) \frac{\partial\left(\mathbf{w}_{\infty}\right)_{2}}{\partial z_{2}} .
\end{aligned}
$$

Hence for every $\varphi \in C_{0}^{1}\left(R ; \mathbb{R}^{2}\right)$

$$
\begin{aligned}
& \int_{C_{\infty}} \mathbf{E}(\varphi) \cdot C\left[\mathbf{E}_{\infty}\right] d \mathbf{z}=(2 \mu+\lambda) \int_{C_{\infty}} e_{\infty}\left(z_{2}\right) \frac{\partial \varphi_{1}}{\partial z_{1}} d \mathbf{z} \\
& +\lambda \int_{C_{\infty}} e_{\infty}\left(z_{2}\right) \frac{\partial \varphi_{2}}{\partial z_{2}} d \mathbf{z} \\
& =(2 \mu+\lambda) \int_{C_{\infty}} \widetilde{e}_{\infty}\left(z_{2}\right) \frac{\partial \varphi_{1}}{\partial z_{1}} d \mathbf{z}+\lambda \int_{C_{\infty}} e_{\infty}\left(z_{2}\right) \frac{\partial \varphi_{2}}{\partial z_{2}} d \mathbf{z} \\
& =\int_{C_{\infty}}\left[(2 \mu+\lambda) \frac{\partial\left(\mathbf{w}_{\infty}\right)_{1}}{\partial z_{1}}+\lambda \frac{\partial\left(\mathbf{w}_{\infty}\right)_{2}}{\partial z_{2}}\right] \frac{\partial \varphi_{1}}{\partial z_{1}} d \mathbf{z} \\
& +\int_{C_{\infty}}\left[\lambda \frac{\partial\left(\mathbf{w}_{\infty}\right)_{1}}{\partial z_{1}}+(2 \mu+\lambda) \frac{\partial\left(\mathbf{w}_{\infty}\right)_{2}}{\partial z_{2}}\right] \frac{\partial \varphi_{2}}{\partial z_{2}} d \mathbf{z} \\
& =\int_{C_{\infty}} \mathbf{E}(\varphi) \cdot C\left[\mathbf{E}\left(\mathbf{w}_{\infty}\right)\right] d \mathbf{z} .
\end{aligned}
$$

We can now proceed exactly as in the previous step with the only difference that we now take

$$
\mathbf{v}_{\infty}:=\mathbf{u}_{\infty}-\frac{1}{\lambda_{\infty}} \mathbf{w}_{\infty} .
$$

Step 6: By Steps 2-5 the estimate (3.58) holds, and we are now in position to prove (3.57). By (3.58) for all $\tau \in\left(0, \tau_{0}\right)$ we have

$$
\begin{aligned}
\int_{C\left(\mathbf{z}_{0}, \tau r\right)}\left(1+|\nabla \mathbf{u}|^{2}\right) d \mathbf{x} & \leq \tau^{2} 16 L r^{2}+c_{1} \tau^{2 \beta} \int_{C\left(\mathbf{z}_{0}, r\right)}\left(1+|\nabla \mathbf{u}|^{2}\right) d \mathbf{x} \\
& \leq\left(16 L+c_{1}\right) \tau^{2 \beta} \int_{C\left(\mathbf{z}_{0}, r\right)}\left(1+|\nabla \mathbf{u}|^{2}\right) d \mathbf{x}
\end{aligned}
$$

for all $0<r<r_{\tau}$. Hence for a fixed $\alpha \in(1 / 2, \beta)$

$$
\left(16 L+c_{1}\right) \tau^{2 \beta}=\left(16 L+c_{1}\right) \tau^{2 \beta-2 \alpha} \tau^{2 \alpha} \leq \tau^{2 \alpha}
$$


provided $\tau_{0}$ is sufficiently small.

Fix $0<r<r_{\tau}$ and find $k \in \mathbb{N}$ such that

$$
\tau^{k+1} r_{\tau} \leq r \leq \tau^{k} r_{\tau}
$$

By iterating (3.65) and by (3.66) we have

$$
\begin{aligned}
\int_{B\left(\mathbf{z}_{0}, r\right) \cap \Omega}|\nabla \mathbf{u}|^{2} d \mathbf{x} & \leq \int_{C\left(\mathbf{z}_{0}, r\right)}\left(1+|\nabla \mathbf{u}|^{2}\right) d \mathbf{x} \leq \int_{C\left(\mathbf{z}_{0}, \tau^{k} r_{\tau}\right)}\left(1+|\nabla \mathbf{u}|^{2}\right) d \mathbf{x} \\
& \leq \tau^{2 \alpha k} \int_{C\left(\mathbf{z}_{0}, r_{\tau}\right)}\left(1+|\nabla \mathbf{u}|^{2}\right) d \mathbf{x} \leq \frac{r^{2 \alpha}}{\left(\tau r_{\tau}\right)^{2 \alpha}} \int_{C\left(\mathbf{z}_{0}, r_{\tau}\right)}\left(1+|\nabla \mathbf{u}|^{2}\right) d \mathbf{x},
\end{aligned}
$$

where we have used the fact that $B\left(\mathbf{z}_{0}, r\right) \cap \Omega \subset C\left(\mathbf{z}_{0}, r\right)$ since $L \geq 1$. This yields (3.57) with

$$
c:=\frac{1}{\left(\tau r_{\tau}\right)^{2 \alpha}} \int_{C\left(\mathbf{z}_{0}, r_{\tau}\right)}\left(1+|\nabla \mathbf{u}|^{2}\right) d \mathbf{x}
$$

and $r_{0}:=r_{\tau}$.

Next we prove that for a local minimizer $(\mathbf{u}, \Omega) \in X$ the upper boundary $\Gamma$ is of class $C^{1}$ away from the finite singular set defined in (3.44) .

Theorem $3.13\left(C^{1}\right.$ Regularity of $\left.\Gamma\right)$ Let $(\mathbf{u}, \Omega) \in X$ be a $\delta$-local minimizer for the functional $F_{\infty}$ defined in (3.1). Then $\Gamma \backslash \Gamma_{\text {sing }}$ is of class $C^{1}$.

Proof. Since $\Gamma_{\text {cuts }}$ is made of segments, it is enough to prove the regularity of $\Gamma \backslash\left(\Gamma_{\text {cusps }} \cup \Gamma_{\text {cuts }}\right)$. Assume by contradiction that $\Gamma$ has a corner at some point $\mathbf{z}_{0} \in \Gamma \backslash\left(\Gamma_{\text {cusps }} \cup \Gamma_{\text {cuts }}\right)$.

By Proposition 3.5 and a standard extension argument we may define $\mathbf{u}$ in a fixed neighborhood of $\mathbf{z}_{0}$ in such a way that for all $0<r<r_{1}$

$$
\int_{B\left(\mathbf{z}_{0}, r\right)}|\nabla \mathbf{u}|^{2} d \mathbf{x} \leq c(L) \int_{B\left(\mathbf{z}_{0}, r\right) \cap \Omega}|\nabla \mathbf{u}|^{2} d \mathbf{x}
$$

for some $r_{1}>0$, and where the constant $c(L)$ is independent of $r$ and depends only on the Lipschitz constant $L$ of the function $g$ in Proposition 3.5.

Moreover, by Proposition 3.1 there exists $\ell_{0}>0$ such that

$$
F_{\infty}(\mathbf{u}, \Omega)=\min \left\{F_{\infty}\left(\mathbf{v}, \Omega_{g}\right)+\ell_{0}|d-| \Omega_{g}^{+}||: \quad\left(\mathbf{v}, \Omega_{g}\right) \in X,\left|\Omega_{g} \Delta \Omega\right| \leq \frac{\delta}{2}\right\},
$$

where $d:=\left|\Omega^{+}\right|$. We recall also that by Proposition 3.5(i) $\Gamma$ admits a left and a right tangent line at $x_{0}$. We are assuming by contradiction that the two tangent lines are distinct and form an angle $0<\vartheta<\pi$. Let us also suppose, to fix the ideas, that $\mathbf{z}_{0} \notin \Gamma_{\text {vert }}$, since the case $\mathbf{z}_{0} \in \Gamma_{\text {vert }}$ can be treated similarly. For $r>0$ (sufficiently small) we denote

$$
\begin{aligned}
& x_{r}^{\prime}:=\max \left\{x \in(a, b): x \leq x_{0} \text { and there exists } y \text { s.t. }(x, y) \in \Gamma \cap \partial B\left(\mathbf{z}_{0}, r\right)\right\}, \\
& x_{r}^{\prime \prime}:=\min \left\{x \in(a, b): x \geq x_{0}, \text { and there exists } y \text { s.t. }(x, y) \in \Gamma \cap \partial B\left(\mathbf{z}_{0}, r\right)\right\},
\end{aligned}
$$

and we let $\left(x_{r}^{\prime}, h\left(x_{r}^{\prime}\right)\right)$ and $\left(x_{r}^{\prime \prime}, h\left(x_{r}^{\prime \prime}\right)\right)$ be the corresponding points on $\Gamma \cap \partial B\left(\mathbf{z}_{0}, r\right)$. Construct $h_{r}$ as the greatest lower semicontinuous function coinciding with $h$ outside $\left[x_{r}^{\prime}, x_{r}^{\prime \prime}\right]$ and with the affine function

$$
x \mapsto h\left(x_{r}^{\prime}\right)+\frac{h\left(x_{r}^{\prime \prime}\right)-h\left(x_{r}^{\prime}\right)}{x_{r}^{\prime \prime}-x_{r}^{\prime}}\left(x-x_{r}^{\prime}\right)
$$

in $\left(x_{r}^{\prime}, x_{r}^{\prime \prime}\right)$. It is easy to see that for $r>0$ sufficiently small $\left(\mathbf{u}, \Omega_{h_{r}}\right)$ is admissible for the penalized minimization problem (3.3). Hence, by (3.68),

$$
F_{\infty}(\mathbf{u}, \Omega) \leq F_{\infty}\left(\mathbf{u}, \Omega_{h_{r}}\right)+\ell_{0}|d-| \Omega_{h_{r}}^{+}|| \leq F_{\infty}\left(\mathbf{u}, \Omega_{h_{r}}\right)+c r^{2},
$$


and, in turn, using the estimates (3.57) and (3.67),

$$
\begin{aligned}
\int_{x_{r}^{\prime}}^{x_{r}^{\prime \prime}} \sqrt{1+\left(h^{\prime}\right)^{2}} d x & \leq \int_{x_{r}^{\prime}}^{x_{r}^{\prime \prime}} \sqrt{1+\left(h_{r}^{\prime}\right)^{2}} d x+c \int_{B\left(\mathbf{z}_{0}, r\right)}|\nabla \mathbf{u}|^{2} d \mathbf{x}+c r^{2} \\
& \leq \int_{x_{r}^{\prime}}^{x_{r}^{\prime \prime}} \sqrt{1+\left(h_{r}^{\prime}\right)^{2}} d x+c r^{2 \alpha}
\end{aligned}
$$

for $r$ small enough. Recall that by Proposition 3.5 the right and the left derivatives $h_{+}^{\prime}$ and $h_{-}^{\prime}$ exist and are continuous in a neighborhood of $x_{0}$. In particular, for $x>x_{0}$ we have $h(x)=$ $h\left(x_{0}\right)+h_{+}^{\prime}\left(x_{0}\right)\left(x-x_{0}\right)+o\left(x-x_{0}\right)$. Since

$$
r=\left|\left(x_{r}^{\prime \prime}, h\left(x_{r}^{\prime \prime}\right)\right)-\left(x_{0}, h\left(x_{0}\right)\right)\right|=\left(x_{r}^{\prime \prime}-x_{0}\right) \sqrt{1+\left(h_{+}^{\prime}\left(x_{0}\right)+\frac{o\left(x_{r}^{\prime \prime}-x_{0}\right)}{x_{r}^{\prime \prime}-x_{0}}\right)^{2}},
$$

we get that

$$
x_{r}^{\prime \prime}=x_{0}+\frac{r}{\sqrt{1+\left(h_{+}^{\prime}\left(x_{0}\right)+\frac{o\left(x_{\prime \prime}^{\prime \prime}-x_{0}\right)}{x_{r}^{\prime \prime}-x_{0}}\right)^{2}}} .
$$

Similarly, we obtain

$$
x_{r}^{\prime}=x_{0}-\frac{r}{\sqrt{1+\left(h_{-}^{\prime}\left(x_{0}\right)+\frac{o\left(x_{r}^{\prime}-x_{0}\right)}{x_{0}-x_{r}^{\prime}}\right)^{2}}} .
$$

Plugging (3.70) and (3.71) in estimate (3.69), dividing both sides by $r$ and letting $r$ go to zero, we immediately get, taking into account the right and left continuity at $x_{0}$ of $h_{+}^{\prime}$ and $h_{-}^{\prime}$ respectively,

$$
2 \leq 2 \sin (\vartheta / 2)
$$

which is impossible.

As an immediate corollary of the previous theorem we have a rigorous proof of the zero contactangle condition between wetting layer and island (see [26] for a discussion on this matter).

Corollary 3.14 (Zero Contact-Angle) Let $(\mathbf{u}, \Omega) \in X$ be a local minimizer for the functional $F_{\infty}$ defined in (3.1). If $\mathbf{z}_{0}=\left(x_{0}, 0\right) \in \Gamma \backslash \Gamma_{\text {sing }}$ then $h^{\prime}\left(x_{0}\right)=0$.

Next we seek to prove $C^{1, \sigma}$ regularity of $\Gamma$ away from the cusp points. To this purpose we need a uniform version of the decay estimate (3.57) .

Theorem 3.15 (Decay Estimate II) Let $(\mathbf{u}, \Omega) \in X$ be a local minimizer for the functional $F_{\infty}$ defined in (3.1). Then for every closed subarc $\Gamma^{\prime} \subset \Gamma \backslash\left(\Gamma_{\text {cusps }} \cup \Gamma_{\text {cuts }}\right)$ and for every $0<\sigma<1$ there exist a constant $c>0$ and a radius $r_{0}$ such that for all $0<r \leq r_{0}$ and for all $\mathbf{z}_{0} \in \Gamma^{\prime}$

$$
\int_{B\left(\mathbf{z}_{0}, r\right) \cap \Omega}|\nabla \mathbf{u}|^{2} d \mathbf{x} \leq c r^{2 \sigma} .
$$

Proof. The proof is very similar to the one of Theorem 3.12 so we only indicate the main changes. We begin by showing that there exist two constants $c>0, \tau_{0} \in(0,1)$ such that for all $\tau \in\left(0, \tau_{0}\right)$ there exists a radius $r_{\tau}>0$ such that for all $0<r \leq r_{\tau}$ and for all $\mathbf{z}_{0} \in \Gamma^{\prime}$

$$
\int_{C\left(\mathbf{z}_{0}, \tau r\right)}|\nabla \mathbf{u}|^{2} d \mathbf{x} \leq c \tau^{2} \int_{C\left(\mathbf{z}_{0}, r\right)}\left(1+|\nabla \mathbf{u}|^{2}\right) d \mathbf{x},
$$

where $C\left(\mathbf{z}_{0}, r\right)$ is defined in (3.59) and $\mathbf{e}_{1}, \mathbf{e}_{2}$ are respectively the unit tangent vector and the normal to the curve $\Gamma$ at $\mathbf{z}_{0}$. 
We fix $c>0, \tau_{0} \in(0,1)$ to be determined later and we assume by contradiction that the corresponding estimate $(3.73)$ is false for some $\tau \in\left(0, \tau_{0}\right)$. Hence we may find a sequence of radii $\left\{r_{n}\right\}$ converging to zero and a sequence $\left\{\mathbf{z}_{n}\right\} \subset \Gamma^{\prime}$ converging to some $\mathbf{z}_{0} \in \Gamma^{\prime}$ such that

$$
\int_{C\left(\mathbf{z}_{n}, \tau r_{n}\right)}|\nabla \mathbf{u}|^{2} d \mathbf{x}>c \tau^{2} \int_{C\left(\mathbf{z}_{n}, r_{n}\right)}\left(1+|\nabla \mathbf{u}|^{2}\right) d \mathbf{x}
$$

Define the sets

$$
C_{n}:=\frac{1}{r_{n}}\left(-\mathbf{z}_{n}+C\left(\mathbf{z}_{n}, r_{n}\right)\right)
$$

Using the fact that, since $\Gamma \backslash\left(\Gamma_{\text {cusps }} \cup \Gamma_{\text {cuts }}\right)$ is of class $C^{1}$, the unit tangent and normal vector to $\Gamma^{\prime}$ vary with continuity one can show that $\chi_{C_{n}}$ converges a.e. to the $\chi_{C_{\infty}}$ where

$$
C_{\infty}:=\left\{s \mathbf{e}_{1}+t \mathbf{e}_{2}:-1<s<1,-4 L<t<g_{\infty}(s)\right\}
$$

with $g_{\infty}(s):=g^{\prime}(0) s$. We rescale accordingly also the function $\mathbf{u}$ by setting

$$
\mathbf{u}_{n}(\mathbf{z}):=\frac{\mathbf{u}\left(\mathbf{z}_{n}+r_{n} \mathbf{z}\right)-\mathbf{a}_{n}}{\lambda_{n} r_{n}},
$$

where $\mathbf{z}:=\frac{\mathbf{x}-\mathbf{z}_{n}}{r_{n}}$,

$$
\mathbf{a}_{n}:=\frac{1}{\left|C\left(x_{n}, r_{n}\right)\right|} \int_{C\left(x_{n}, r_{n}\right)} \mathbf{u} d \mathbf{z}, \quad \lambda_{n}^{2}:=\frac{1}{\left|C\left(x_{n}, r_{n}\right)\right|} \int_{C\left(x_{n}, r_{n}\right)}|\nabla \mathbf{u}|^{2} d \mathbf{z} .
$$

As in the proof of Step 1 of Theorem 3.12 we may assume that $\mathbf{u}_{n}$ (extended to the rectangle $R$ ) weakly converges to some function $\mathbf{u}_{\infty} \in W^{1,2}\left(R ; \mathbb{R}^{2}\right)$ and that

$$
\lambda_{n} \rightarrow \lambda_{\infty} \in(0, \infty] .
$$

The proof of Step 2 of Theorem 3.12 continues to hold while Step 3 can be simplified.

Indeed, if $\lambda_{\infty}=\infty$ then $\mathbf{u}_{\infty}$ is a weak solution of the problem

$$
\begin{aligned}
\mu \Delta \mathbf{u}_{\infty}+(\lambda+\mu) \nabla\left(\operatorname{div} \mathbf{u}_{\infty}\right)=\mathbf{0} & \text { in } C_{\infty}, \\
{\left[\mu\left(\nabla \mathbf{u}_{\infty}+\nabla \mathbf{u}_{\infty}^{T}\right)+\lambda\left(\operatorname{div} \mathbf{u}_{\infty}\right) I\right] \nu=\mathbf{0} } & \text { on } \Gamma_{g_{\infty}} .
\end{aligned}
$$

Therefore by Theorem 3.7 we have that if $0<r<\frac{1}{2}$ then

$$
\int_{B(\mathbf{0}, r) \cap C_{\infty}}\left|\nabla \mathbf{u}_{\infty}\right|^{2} d \mathbf{z} \leq C r^{2} \sup _{B(0, r) \cap C_{\infty}}\left|\nabla \mathbf{u}_{\infty}\right|^{2} \leq C r^{2} \int_{B(\mathbf{0}, 1) \cap C_{\infty}}\left|\nabla \mathbf{u}_{\infty}\right|^{2} d \mathbf{z} \leq C r^{2} .
$$

We may now proceed as before to obtain a contradiction.

If $\lambda_{\infty}<\infty$ then as in Step 4 of Theorem 3.12 we set

$$
\mathbf{v}_{\infty}(\mathbf{z}):=\mathbf{u}_{\infty}(\mathbf{z})-\frac{\left(e_{0} z_{1}, 0\right)}{\lambda_{\infty}}
$$

Then $\mathbf{v}_{\infty}$ is a weak solution of the problem

$$
\begin{aligned}
\mu \Delta \mathbf{v}_{\infty}+(\lambda+\mu) \nabla\left(\operatorname{div} \mathbf{v}_{\infty}\right)=\mathbf{0} & \text { in } C_{\infty} \\
{\left[\mu\left(\nabla \mathbf{v}_{\infty}+\nabla \mathbf{v}_{\infty}^{T}\right)+\lambda\left(\operatorname{div} \mathbf{v}_{\infty}\right) I\right] \nu=\mathbf{0} } & \text { on } \Gamma_{g_{\infty}}
\end{aligned}
$$

and thus, as in the previous step, for all $0<r<\frac{1}{2}$ we have

$$
\int_{B(\mathbf{0}, r) \cap C_{\infty}}\left|\nabla \mathbf{v}_{\infty}\right|^{2} d \mathbf{z} \leq C r^{2}
$$

and the rest of the proof is analogous.

Steps 5 and 6 are also similar, we omit the details.

We are now in a position to show that $\Gamma$ is of class $C^{1, \sigma}$ away from the finite singular set $\Gamma_{\text {sing }}$. 
Theorem $3.16\left(C^{1, \sigma}\right.$ Regularity of $\left.\Gamma\right)$ Let $(\mathbf{u}, \Omega) \in X$ be a $\delta$-local minimizer for the functional $F_{\infty}$ defined in (3.1). Then $\Gamma \backslash \Gamma_{\text {sing }}$ is of class $C^{1, \sigma}$ for all $0<\sigma<\frac{1}{2}$.

Proof. As in the proof of Theorem 3.13 it is enough to consider $\Gamma \backslash\left(\Gamma_{\text {cusps }} \cup \Gamma_{\text {cuts }}\right)$. Fix a closed subarc $\Gamma^{\prime} \subset \Gamma \backslash\left(\Gamma_{\text {cusps }} \cup \Gamma_{\text {cuts }}\right), \frac{1}{2}<\sigma_{0}<1$, and a point $\mathbf{z}_{0}=\left(x_{0}, y_{0}\right)$ in $\Gamma^{\prime}$. As in the proof of Theorem 3.13, for all $0<r \leq r_{1}$ we may extend $\mathbf{u}$ to the ball $B\left(\mathbf{z}_{0}, r\right)$ in such a way that (3.67) holds. Moreover, since $\Gamma \backslash\left(\Gamma_{\text {cusps }} \cup \Gamma_{\text {cuts }}\right)$ is of class $C^{1}$, we may assume that constants $r_{1}, L$ and $c(L)$ in (3.67) are independent of $\mathbf{z}_{0}$. Hence also by (3.72) there exist $c, r_{0}>0$ indepedent of $\mathbf{z}_{0}$ such that

$$
\int_{B\left(\mathbf{z}_{0}, r\right)}|\nabla \mathbf{u}|^{2} d \mathbf{x} \leq c r^{2 \sigma_{0}}
$$

for all $0<r<r_{0}$.

Owing to the $C^{1}$ regularity and taking $r_{0}$ smaller (and again independently of $\mathbf{z}_{0}$ ), we can also assume that for all $0<r<r_{0}$, the curve $\Gamma$ crosses transversally $\partial B\left(\mathbf{z}_{0}, r\right)$ at exactly two points.

Let $\left(x_{r}^{\prime}, y_{r}^{\prime}\right)$ and $\left(x_{r}^{\prime \prime}, y_{r}^{\prime \prime}\right)$ be two points in $\partial B\left(\mathbf{z}_{0}, r\right) \cap \Gamma$. We redefine $\Gamma$ inside the ball $B\left(\mathbf{z}_{0}, r\right)$ as the polygonal path of length $2 r$ connecting $\left(x_{r}^{\prime}, y_{r}^{\prime}\right)$ with $\mathbf{z}_{0}$ and $\mathbf{z}_{0}$ with $\left(x_{r}^{\prime \prime}, y_{r}^{\prime \prime}\right)$ and we denote the corresponding function by $h_{r}$.

Using Proposition 3.1 and the fact that if $r_{0}$ is small enough the new pair $\left(\mathbf{u}, \Omega_{h_{r}}\right)$ is admissible for problem (3.3), we can estimate

$$
F_{\infty}(\mathbf{u}, \Omega) \leq F_{\infty}\left(\mathbf{u}, \Omega_{h_{r}}\right)+\ell_{0}|d-| \Omega_{h_{r}}^{+}|| \leq F_{\infty}\left(\mathbf{u}, \Omega_{h_{r}}\right)+c r^{2},
$$

which, together with (3.74), yields

$$
\mathcal{H}^{1}\left(\Gamma_{h} \cap B(\mathbf{z}, r)\right)-2 r \leq c \int_{B(\mathbf{z}, r)}|\nabla \mathbf{u}|^{2} d \mathbf{x}+c r^{2} \leq c_{1} r^{2 \sigma_{0}} .
$$

Note that the previous inequality holds for all $\mathbf{z} \in \Gamma^{\prime}$ and $0<r<r_{0}$, with $c_{1}$ independent of $\mathbf{z}$ and $r$. It follows that $\Gamma^{\prime}$ is of class $C^{1, \sigma}$, where $\sigma:=\sigma_{0}-\frac{1}{2}$ (see Proposition 6.4 and the proof of Theorem 6.1 in [3]).

In view of the previous theorem and Remark 3.6 we can partition $(a, b)$ as

$$
a=: x_{0}<x_{1}<\ldots<x_{m-1}<x_{m}:=b
$$

in such a way $\Gamma \cap\left(\left(x_{i-1}, x_{i}\right) \times[0, \infty)\right)$ is of class $C^{1, \sigma}$ for all $0<\sigma<\frac{1}{2}$ for each $i=1, \ldots, m$.

Theorem $3.17\left(C^{1, \sigma}\right.$ Regularity of $\left.\mathbf{u}\right)$ Let $(\mathbf{u}, \Omega) \in X$ be a $\delta$-local minimizer for the functional $F_{\infty}$ defined in (3.1). Then for any $\left[a^{\prime}, b^{\prime}\right] \subset\left(x_{i-1}, x_{i}\right), i=1, \ldots, m$, we have that $\mathbf{u}-\mathbf{w}_{\infty} \in$ $C^{1, \sigma_{1}}\left(\bar{\Omega} \cap\left(\left[a^{\prime}, b^{\prime}\right] \times \mathbb{R}\right) ; \mathbb{R}^{2}\right)$, for some $\sigma_{1}>0$, where

$$
\mathbf{w}_{\infty}(\mathbf{x}):=e_{0}\left(x,-\min \left\{0, \frac{\lambda y}{2 \mu+\lambda}\right\}\right) .
$$

In particular, $\mathbf{u} \in C^{1, \sigma_{1}}\left(\bar{\Omega} \cap\left(\left[a^{\prime}, b^{\prime}\right] \times \mathbb{R} \backslash\{0\}\right) ; \mathbb{R}^{2}\right)$.

Proof. As shown in Step 5 of the proof of Theorem 3.12 the function $\mathbf{u}-\mathbf{w}_{\infty}$ satisfies

$$
\int_{\Omega} \mathbf{E}(\varphi) \cdot C\left[\mathbf{E}\left(\mathbf{u}-\mathbf{w}_{\infty}\right)\right] d \mathbf{x}=0
$$

for every $\varphi \in C_{c}^{1}\left(\left(a^{\prime}, b^{\prime}\right) \times \mathbb{R} ; \mathbb{R}^{2}\right)$. We now argue as in the proof of Theorem 7.53 in [2] to which we refer for some details of the proof that will be omitted here.

By straightening the boundary $\Gamma$ with a diffeomorphism of class $C^{1, \sigma}$ for every $0<\sigma<\frac{1}{2}$, the function $\mathbf{u}-\mathbf{w}_{\infty}$ is transformed in a $H^{1}$ function $\mathbf{v}$ satisfying a linear system of the form

$$
\int_{B^{-}} \nabla \varphi A(\mathbf{x}) D \mathbf{v} d \mathbf{x}=0
$$


for all $\varphi \in C^{1}\left(B^{-} ; \mathbb{R}^{2}\right)$ vanishing in a neighborhood of $\left.\partial B^{-} \cap\{y<0\}\right)$, where $B^{-}$is a half ball centered at the origin contained in $\{y<0\}$ and the coefficients $A_{i, j}^{h, k}$ of the matrix $A$ are of class $C^{0, \sigma}$ for $0<\sigma<\frac{1}{2}$. Moreover, since $\lambda+\mu>0$ and $\mu>0$, the matrix $A$ satisfies the strong Legendre-Hadamard ellipticity condition. Let us extend the function $\mathbf{v}$ to the whole ball $B$ by setting $\mathbf{v}(x, y)=\mathbf{v}(x,-y)$ for $y>0$. It is easy to check that the new function $\mathbf{v}$ satisfies the equation

$$
\int_{B} \nabla \varphi A(x) \nabla \mathbf{v} d \mathbf{x}=0
$$

for all $\varphi \in C_{0}^{1}\left(B ; \mathbb{R}^{2}\right)$, where the coefficients of $A$ are also extended to the upper ball $B^{+}$by setting, for $y>0$,

$$
A_{i, j}^{h, k}(x, y)=\left\{\begin{array}{cc}
A_{i, j}^{h, k}(x,-y) & \text { if } h=k=1 \text { or } h=k=2 \\
-A_{i, j}^{h, k}(x,-y) & \text { otherwise. }
\end{array}\right.
$$

Let us fix $\left(x_{0}, 0\right) \in B$ and $B\left(\left(x_{0}, 0\right), R\right) \subset B$. We denote by $\mathbf{w}$ the weak solution of the Dirichlet problem

$$
\left\{\begin{array}{cl}
\int_{B\left(\left(x_{0}, 0\right), R\right)} \nabla \varphi \bar{A} \nabla \mathbf{w} d \mathbf{x}=0, & \\
\mathbf{w}=\mathbf{v} & \text { on } \partial B\left(\left(x_{0}, 0\right), R\right)
\end{array}\right.
$$

where

$$
\bar{A}(\mathbf{x})= \begin{cases}A^{+}=\frac{1}{\left|B^{+}\right|} \int_{B^{+}} A(\mathbf{x}) d \mathbf{x} & \text { in } B^{+} \\ A^{-}=\frac{1}{\left|B^{-}\right|} \int_{B^{-}} A(\mathbf{x}) d \mathbf{x} & \text { in } B^{-} .\end{cases}
$$

By the standard difference quotient argument and Korn's inequality, one can show that $\frac{\partial \mathbf{w}}{\partial x}$ is a $W_{\text {loc }}^{1,2}$ weak solution of

$$
\int_{B\left(\left(x_{0}, 0\right), R\right)} \nabla \varphi \bar{A} \nabla\left(\frac{\partial \mathbf{w}}{\partial x}\right) d \mathbf{x}=0 .
$$

Therefore, by standard regularity estimates, we get that for every ball $B(\mathbf{z}, \varrho) \subset B\left(\left(x_{0}, 0\right), R\right)$

$$
\int_{B(\mathbf{z}, \varrho / 2)}\left|\nabla\left(\frac{\partial \mathbf{w}}{\partial x}\right)\right|^{2} d \mathbf{x} \leq \frac{c}{\varrho^{2}} \int_{B(\mathbf{z}, \varrho)}\left|\left(\frac{\partial \mathbf{w}}{\partial x}\right)-\left(\frac{\partial \mathbf{w}}{\partial x}\right)_{\mathbf{z}, \varrho}\right|^{2} d \mathbf{x},
$$

where $\left(\frac{\partial \mathbf{w}}{\partial x}\right)_{\mathbf{z}, \varrho}$ denotes the average of the function $\frac{\partial \mathbf{w}}{\partial x}$ in the ball $B_{\varrho}(\mathbf{z})$. From inequality $(3.77)$ and Poincaré inequality we get

$$
\frac{1}{|B(\mathbf{z}, \varrho / 2)|} \int_{B(\mathbf{z}, \varrho / 2)}\left|\nabla\left(\frac{\partial \mathbf{w}}{\partial x}\right)\right|^{2} d \mathbf{x} \leq c\left(\frac{1}{|B(\mathbf{z}, \varrho)|} \int_{B(\mathbf{z}, \varrho)}\left|\nabla\left(\frac{\partial \mathbf{w}}{\partial x}\right)\right|^{2} d \mathbf{x}\right)^{2} .
$$

From this inequality, by using Gehring's lemma (see for instance [15], Proposition 1.1, Chap. V) we get that $\frac{\partial \mathbf{w}}{\partial x} \in W_{\text {loc }}^{1, p}\left(B ; \mathbb{R}^{2}\right)$ for some $p>2$ and for all $B(\mathbf{z}, \varrho) \subset B\left(\left(x_{0}, 0\right), R\right)$

$$
\left(\frac{1}{|B(\mathbf{z}, \varrho / 2)|} \int_{B(\mathbf{z}, \varrho / 2)}\left|\nabla\left(\frac{\partial \mathbf{w}}{\partial x}\right)\right|^{p} d \mathbf{x}\right)^{1 / p} \leq c_{1}\left(\frac{1}{|B(\mathbf{z}, \varrho)|} \int_{B(\mathbf{z}, \varrho)}\left|\nabla\left(\frac{\partial \mathbf{w}}{\partial x}\right)\right|^{2} d \mathbf{x}\right)^{1 / 2}
$$

for some constant $c_{1}$ independent of the ball $B(\mathbf{z}, \varrho)$. Note that, since $p>2$, we may conclude that $\frac{\partial \mathbf{w}}{\partial x}$ is locally $\gamma$-Hölder continuous for $\gamma=1-2 / p$. Moreover, from Sobolev Embedding Theorem and (3.78) we obtain that

$$
\max _{B(\mathbf{z}, \varrho / 2)}\left|\frac{\partial \mathbf{w}}{\partial x}\right|^{2} \leq \frac{c}{|B(\mathbf{z}, \varrho)|} \int_{B(\mathbf{z}, \varrho)}\left|\frac{\partial \mathbf{w}}{\partial x}\right|^{2} d \mathbf{x}+\frac{c \varrho^{2}}{|B(\mathbf{z}, \varrho)|} \int_{B(\mathbf{z}, \varrho)}\left|\nabla\left(\frac{\partial \mathbf{w}}{\partial x}\right)\right|^{2} d \mathbf{x} .
$$


This inequality and (3.77) yield

$$
\max _{B(\mathbf{z}, \varrho / 2)}\left|\frac{\partial \mathbf{w}}{\partial x}\right|^{2} \leq \frac{c}{|B(\mathbf{z}, 2 \varrho)|} \int_{B(\mathbf{z}, 2 \varrho)}\left|\frac{\partial \mathbf{w}}{\partial x}\right|^{2} d \mathbf{x} .
$$

Denoting by $\left[\frac{\partial \mathbf{w}}{\partial x}\right]_{\gamma, \mathbf{z}, r}$ the Hölder seminorm of $\frac{\partial \mathbf{w}}{\partial x}$ in the ball $B(\mathbf{z}, r)$, from Sobolev embedding theorem, (3.78) and (3.77) we get that if $B(\mathbf{z}, 4 r) \subset B\left(\left(x_{0}, 0\right), R\right)$ and $\varrho<r$

$$
\begin{aligned}
\int_{B(\mathbf{z}, \varrho)}\left|\frac{\partial \mathbf{w}}{\partial x}-\left(\frac{\partial \mathbf{w}}{\partial x}\right)_{\mathbf{z}, \varrho}\right|^{2} d \mathbf{x} \leq c \varrho^{2+2 \gamma}\left[\frac{\partial \mathbf{w}}{\partial x}\right]_{\gamma, \mathbf{z}, r}^{2} \\
\leq c \varrho^{2+2 \gamma} r^{4 / p}\left(\frac{c}{|B(\mathbf{z}, r)|} \int_{B(\mathbf{z}, r)}\left|\nabla\left(\frac{\partial \mathbf{w}}{\partial x}\right)\right|^{p} d \mathbf{x}\right)^{2 / p} \\
\leq c \varrho^{2+2 \gamma} r^{\frac{4}{p}-2} \int_{B(\mathbf{z}, 2 r)}\left|\nabla\left(\frac{\partial \mathbf{w}}{\partial x}\right)\right|^{2} d \mathbf{x} \\
\leq c\left(\frac{\varrho}{r}\right)^{2+2 \gamma} \int_{B(\mathbf{z}, 4 r)}\left|\frac{\partial \mathbf{w}}{\partial x}-\left(\frac{\partial \mathbf{w}}{\partial x}\right)_{\mathbf{z}, 4 r}\right|^{2} d \mathbf{x} .
\end{aligned}
$$

Following the proof of Theorem 7.53 in [2], we introduce the vector $\bar{D}_{c} \mathbf{w}$ whose components are

$$
\left(\bar{D}_{c} \mathbf{w}\right)_{j}=\sum_{h=1}^{2} \sum_{i=1}^{2} \bar{A}_{i j}^{h 2} \frac{\partial w_{i}}{\partial x_{h}}
$$

for $j=1,2$, where for convenience we used the notation $x_{1}=x, x_{2}=y$. From the equation satisfied by $\mathbf{w}$ we get that for $j=1,2$

$$
\frac{\partial\left(\left(\bar{D}_{c} \mathbf{w}\right)_{j}\right)}{\partial x_{2}}=-\sum_{h=1}^{2} \sum_{i=1}^{2} \bar{A}_{i j}^{h 1} \frac{\partial^{2} w_{i}}{\partial x_{1} \partial x_{h}} .
$$

Therefore we may conclude that $\nabla\left(\bar{D}_{c} \mathbf{w}\right)$ is locally in $L^{2}$ in $B\left(\left(x_{0}, 0\right), R\right)$ and

$$
\left|\nabla\left(\bar{D}_{c} \mathbf{w}\right)\right| \leq c\left|\nabla\left(\frac{\partial \mathbf{w}}{\partial x_{1}}\right)\right|
$$

From now on the proof proceeds exactly as in Theorem 7.53 in [2] with the conclusion that $\nabla \mathbf{v}$ is locally $\sigma$-Hölder continuous for all $\sigma<\min \left\{\gamma, \frac{1}{2}\right\}$.

We will finally prove that $\Gamma$ is analytic outside a bigger singular set $\Sigma$. Consider the contact set $Z:=\Gamma \cap\{y=0\}$ and its inner regularization $Z_{\mathrm{reg}}:=\overline{\stackrel{\circ}{\circ}}$, where the interior part and the closure are with respect to the relative topology. We set

$$
\Sigma:=\Gamma_{\text {cusp }} \cup \Gamma_{\text {vert }} \cup \Gamma_{\text {cuts }} \cup Z_{\text {reg }},
$$

where, we recall, $\Gamma_{\text {cusp }}$ and $\Gamma_{\text {vert }}$ are the sets defined in (2.14) and (3.43), respectively. Note that by construction $\Sigma$ is a closed set.

Theorem 3.18 (Analyticity) Let $(\mathbf{u}, \Omega) \in X$ be a $\delta$-local minimizer for the functional $F_{\infty}$. Then $\Gamma \backslash \Sigma$ is analytic and satisfies the Euler-Lagrange equation

$$
\gamma_{\text {film }} \operatorname{curv} \Gamma(\mathbf{z})=W\left(\mathbf{E}(\mathbf{u})(\mathbf{z})-\mathbf{E}_{0}(y)\right)+\lambda_{0} \quad \text { for all } \mathbf{z} \in \Gamma \backslash \Sigma,
$$

where $\lambda_{0}$ is a suitable Lagrange multiplier and $\operatorname{curv} \Gamma(\mathbf{z})$ denotes the curvature of $\Gamma$ at the point $\mathbf{z}$. 
Proof. Consider the relatively open subset $\Gamma \backslash(\Sigma \cup Z)$ and denote by $A$ its projection on the $x$-axis. By considering variations in $h$ of the form $h+\varepsilon \varphi$, where $\varphi$ has compact support in $A$ and $\int_{A} \varphi d x=0$, we easily get that $\Gamma \backslash(\Sigma \cup Z)$ is weak solution of (3.79). From the previous theorem and this equation we immediately obtain that $\Gamma_{\text {graph }} \backslash(\Sigma \cup Z)$ is of class $C^{2, \sigma}$ for some $\sigma>0$ and thus it is a classical solution of (3.79). Since every point of $\Gamma \backslash \Sigma$ is in the closure of $\Gamma \backslash(\Sigma \cup Z)$, by approximation we see that (3.79) is satisfied classically at every point of $\Gamma \backslash \Sigma$.

Let $\mathbf{w}_{\infty}$ be the function introduced in Theorem 3.17, set $\tilde{\mathbf{u}}:=\mathbf{u}-\mathbf{w}_{\infty}$, and denote by $\mathbf{E}_{\infty}$ the constant value of $\mathbf{E}\left(\mathbf{w}_{\infty}\right)$ on the half space $\{y>0\}$. Taking into account $(3.75)$, it is easy to see that the pair $(\tilde{\mathbf{u}}, \Gamma \backslash \Sigma)$ is a $C^{2}$ solution of the following overdetermined system:

$$
\begin{gathered}
\operatorname{div} C[\mathbf{E}(\tilde{\mathbf{u}})]=0 \quad \text { in } \Omega, \\
C[\mathbf{E}(\tilde{\mathbf{u}})] \cdot \nu=0 \quad \text { on } \Gamma \backslash \Sigma, \\
\gamma_{\text {film }} \operatorname{curv} \Gamma=W(\mathbf{E}(\tilde{\mathbf{u}}))+\left(\mathbf{E}_{\infty}-e_{0} \mathbf{i} \otimes \mathbf{i}\right) \cdot C[\mathbf{E}(\tilde{\mathbf{u}})]+W\left(\mathbf{E}_{\infty}-e_{0} \mathbf{i} \otimes \mathbf{i}\right)+\lambda_{0} \quad \text { on } \Gamma \backslash \Sigma .
\end{gathered}
$$

A standard bootstrap argument now yields that $\Gamma \backslash \Sigma$ is of class $C^{\infty}$ and that $\mathbf{u}-\mathbf{w}_{\infty}$ is $C^{\infty}$ up to $\Gamma \backslash \Sigma$. The analytic regularity follows from Theorem 4.9 and the remarks at the end of Section 4.2 in $[19]$.

Remark 3.19 Denote by $\pi\left(\Sigma \backslash Z_{\text {reg }}\right)$ the projection of $\Sigma \backslash Z_{\text {reg }}$ on the $x$-axis. By the definition of $\Sigma$ and the structure of $\Gamma$ it is easy to see that $\pi\left(\Sigma \backslash Z_{\text {reg }}\right)$ is contained in the set where $h$ is non differentiable. Hence $\mathcal{L}^{1}\left(\pi\left(\Sigma \backslash Z_{\text {reg }}\right)\right)=0$. In particular if we set

$$
U:=\{x \in(a, b): h(x)>0\},
$$

it follows from Theorem 3.18 that $h$ is analytic on an open set of full measure in $U$.

We conclude by proving the following corollary on the structure of the contact set $Z$.

Corollary 3.20 (Contact Set) Let $(\mathbf{u}, \Omega) \in X$ be a $\delta$-local minimizer for the functional $F_{\infty}$ and let $Z=\Gamma \cap\{y=0\}$ be the contact set of $\Gamma$ with the $x$-axis. Then for all open intervals $I \subset(a, b)$ the set $(I \times\{0\}) \cap Z$ is either discrete or with nonempty relative interior part.

Proof. Assume by contradiction that $(I \times\{0\}) \cap Z$ has no relative interior part and admits a cluster point $\mathbf{z}_{0}=\left(x_{0}, 0\right)$. Taking into account the definition of $\Sigma$ and the structure of $\Gamma$ it is easy to see that under these circumstances $\Sigma \cap\left(\left(x_{0}-\varepsilon, x_{0}+\varepsilon\right) \times \mathbb{R}\right)=\emptyset$ if $\varepsilon$ is small enough. It follows from Theorem 3.18 that $h$ is analytic in $\left(x_{0}-\varepsilon, x_{0}+\varepsilon\right)$ and its zero-set has a cluster point at $x_{0}$, a contradiction.

Remark 3.21 It would be interesting to show that $Z$ is in fact the finite union of (possibly degenerate) closed intervals.

\section{Appendix: Korn's Inequalities}

We begin by recalling a classical version of Korn's inequality which may be found in [23], [24].

Theorem 4.1 (Korn's inequality I) Let $M>0$ and let $\Omega \subset \mathbb{R}^{N}$ be an open bounded domain starshaped with respect to a given ball $B\left(\mathbf{x}_{0}, r\right) \subset \Omega$ and such that $\operatorname{diam} \Omega \leq M$. Then there exists a constant $c=c(p, N, r, M)>0$ such that for all $\mathbf{u} \in W^{1, p}\left(\Omega ; \mathbb{R}^{N}\right), p>1$,

$$
\int_{\Omega}|\nabla \mathbf{u}|^{p} d \mathbf{x} \leq c\left(\int_{\Omega}|\mathbf{u}|^{p} d \mathbf{x}+\int_{\Omega}|\mathbf{E}(\mathbf{u})|^{p} d \mathbf{x}\right) .
$$


As a consequence of this theorem we establish the following Korn-type inequality for subgraphs of Lipschitz functions.

Theorem 4.2 (Korn's Inequality II: subgraphs of Lipschitz functions) Let $B_{N-1}(\mathbf{0}, 1)$ be the unit ball in $\mathbb{R}^{N-1}$ and let $h: B_{N-1}(\mathbf{0}, 1) \rightarrow[-L, L]$ be a Lipschitz function with $\operatorname{Lip} h \leq L$ for some $L>0$. Define

$$
R_{h}:=\left\{\left(\mathbf{x}^{\prime}, x_{N}\right) \in \mathbb{R}^{N-1} \times \mathbb{R}: \mathbf{x}^{\prime} \in B_{N-1}(\mathbf{0}, 1),-4 L<x_{N}<h\left(\mathbf{x}^{\prime}\right)\right\} .
$$

Then there exists a constant $c$ depending only on $N, p$, and $L$ such that

$$
\int_{R_{h}}|\nabla \mathbf{u}|^{p} d \mathbf{x} \leq c\left(\int_{R_{h}}|\mathbf{u}|^{p} d \mathbf{x}+\int_{R_{h}}|\mathbf{E}(\mathbf{u})|^{p} d \mathbf{x}\right)
$$

for all $\mathbf{u} \in W^{1, p}\left(R_{h} ; \mathbb{R}^{N}\right), p>1$. Moreover for any ball $B$ compactly contained in $B_{N-1}(\mathbf{0}, 1) \times$ $(-4 L,-3 L)$ there exists a constant $c_{1}$ depending only on $N, p, L$ and on the radius of $B$ such that

$$
\int_{R_{h}}|\nabla \mathbf{u}|^{p} d \mathbf{x} \leq c_{1} \int_{R_{h}}|\mathbf{E}(\mathbf{u})|^{p} d \mathbf{x}
$$

for all $\mathbf{u} \in W^{1, p}\left(R_{h} ; \mathbb{R}^{N}\right)$ with

$$
\int_{B}\left(\nabla \mathbf{u}-(\nabla \mathbf{u})^{T}\right) d \mathbf{x}=\mathbf{0}
$$

Proof. In view of the previous theorem, to prove the first part of the statement it is enough to show that $R_{h}$ is starshaped with respect to any ball $B$ compactly contained in $B_{N-1}(\mathbf{0}, 1) \times$ $(-4 L,-3 L)$. To this aim, let $\mathbf{x}=\left(\mathbf{x}^{\prime}, x_{N}\right) \in R_{h}$ and $\mathbf{y} \in B$. If $x_{N} \leq-L$ then the segment joining $\mathbf{x}$ with $\mathbf{y}$ is clearly contained in $R_{h}$. If $x_{N}>-L$ then the straight line passing through $\mathbf{x}$ and $\mathbf{y}$ has slope greater than $L$ therefore it cannot intersect the graph of the function $h$ in more than one point, that is, the segment joining $\mathbf{x}$ with $\mathbf{y}$ is contained in $R_{h}$.

To prove the second part of the theorem fix a ball $B$ contained in $B_{N-1}(\mathbf{0}, 1) \times(-4 L,-3 L)$. Clearly it suffices to prove (4.1) for all functions $\mathbf{u} \in W^{1, p}\left(R_{h} ; \mathbb{R}^{N}\right)$ which satisfy (4.2) and

$$
\int_{B} \mathbf{u} d \mathbf{x}=\mathbf{0}
$$

By the first part it will be enough to prove that there exists a constant $c_{2}$ depending only on $L$ and $B$ such that

$$
\int_{R_{h}}|\mathbf{u}|^{p} d \mathbf{x} \leq c_{2} \int_{R_{h}}|\mathbf{E}(\mathbf{u})|^{p} d \mathbf{x}
$$

for all $\mathbf{u} \in W^{1, p}\left(R_{h} ; \mathbb{R}^{N}\right)$ satisfying (4.2). Assume by contradiction that (4.3) fails. Then there exist a sequence of functions $\left\{h_{n}\right\}$ as in the statement and a sequence $\left\{\mathbf{u}_{n}\right\}$ of functions with $\mathbf{u}_{n} \in W^{1, p}\left(R_{h_{n}} ; \mathbb{R}^{N}\right)$ such that

$$
\int_{B} \mathbf{u}_{n} d \mathbf{x}=\mathbf{0}, \quad \int_{B}\left(\nabla \mathbf{u}_{n}-\left(\nabla \mathbf{u}_{n}\right)^{T}\right) d \mathbf{x}=\mathbf{0}
$$

and

$$
\int_{R_{h_{n}}}\left|\mathbf{u}_{n}\right|^{p} d \mathbf{x}=1, \quad \lim _{n \rightarrow \infty} \int_{R_{h_{n}}}\left|\mathbf{E}\left(\mathbf{u}_{n}\right)\right|^{p} d \mathbf{x}=0 .
$$

Since $\operatorname{Lip} h_{n} \leq L$ up to a subsequence we may assume that $\left\{h_{n}\right\}$ converges uniformly to a function $h_{\infty}$ with $\operatorname{Lip} h_{\infty} \leq L$. By the first part of the theorem we have that

$$
\sup _{n} \int_{R_{h_{n}}}\left|\nabla \mathbf{u}_{n}\right|^{p} d \mathbf{x} \leq c .
$$


A straightforward extension argument by reflection allows us to extend the functions $\mathbf{u}_{n}$ to the cylinder $C_{L}:=B_{N-1}(\mathbf{0}, 1) \times(-4 L, 4 L)$ in such a way that the resulting functions, still denoted by $\mathbf{u}_{n}$, are equibounded in $W^{1, p}\left(C_{L} ; \mathbb{R}^{N}\right)$. Hence, without loss of generality, we may assume that the sequence $\left\{\mathbf{u}_{n}\right\}$ converges weakly in $W^{1, p}\left(C_{L} ; \mathbb{R}^{N}\right)$ to some function $\mathbf{u}_{\infty}$. We claim that

$$
\int_{R_{h_{\infty}}}\left|\mathbf{u}_{\infty}\right|^{p} d \mathbf{x}=1
$$

Indeed, since $\left\{h_{n}\right\}$ converges uniformly to $h_{\infty}$ it is clear that $\chi_{R_{h_{n}}}$ converges to $\chi_{R_{h_{\infty}}}$ pointwise a.e. in $C_{L}$. Hence by Lebesgue Dominated Convergence Theorem $\left\{\mathbf{u}_{n} \chi_{R_{h_{n}}}\right\}$ converges in $L^{p}$ to $\mathbf{u}_{\infty} \chi_{R_{h_{\infty}}}$. The claim now follows from (4.5).

Moreover, by lower semicontinuity, it is easy to see that

$$
\int_{R_{h_{\infty}}}\left|\mathbf{E}\left(\mathbf{u}_{\infty}\right)\right|^{p} d \mathbf{x} \leq \lim _{n \rightarrow \infty} \int_{R_{h_{n}}}\left|\mathbf{E}\left(\mathbf{u}_{n}\right)\right|^{p} d \mathbf{x}=0
$$

and so $\mathbf{E}\left(\mathbf{u}_{\infty}\right)=\mathbf{0}$ a.e. in $R_{h_{\infty}}$. Since the domain $R_{h_{\infty}}$ is connected this implies that $\mathbf{u}_{\infty}(\mathbf{x})=$ $\mathbf{a}+\mathbf{B x}$ for some constant $\mathbf{a} \in \mathbb{R}^{N}$ and some skew-symmetric matrix $\mathbf{B} \in \mathbb{R}^{N \times N}$. On the other hand, it follows from (4.4) that

$$
\int_{B} \mathbf{u}_{\infty} d \mathbf{x}=\mathbf{0}, \quad \int_{B}\left(\nabla \mathbf{u}_{\infty}-\left(\nabla \mathbf{u}_{\infty}\right)^{T}\right) d \mathbf{x}=\mathbf{0}
$$

and so $\mathbf{a}=\mathbf{0}$ and $\mathbf{B}=\mathbf{0}$, which contradicts (4.6).

We next extend the above theorem to a different class of two-dimensional domains which appear in the proof of Proposition 3.1.

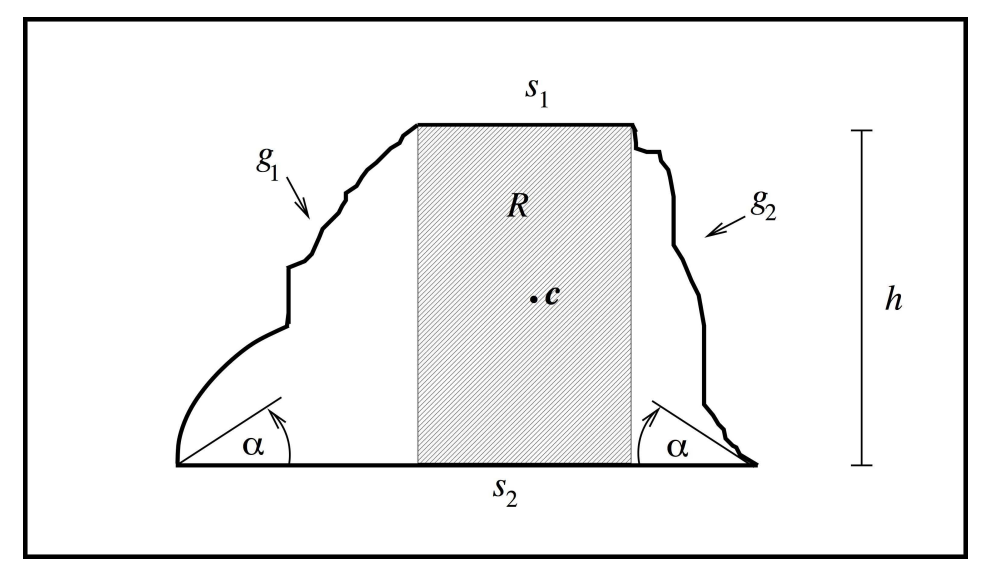

Figure 2: A generalized trapezoid.

A generalized trapezoid $T \subset \mathbb{R}^{2}$ is the open region enclosed by two parallel (horizontal) straight line segments $s_{1}, s_{2}$ and a pair $\gamma_{1}, \gamma_{2}$ of arcs connecting the endpoints of $s_{1}$ and $s_{2}$ with the following properties (see the picture above):

(i) assuming without loss of generality that the upper side $s_{1}$ is shorter than $s_{2}$ and denoting by $\pi\left(s_{1}\right)$ the projection of $s_{1}$ on the straight line containing $s_{2}$, there holds $\pi\left(s_{1}\right) \subset s_{2}$;

(ii) each $\gamma_{i}, i=1,2$, is either the extended graph of a monotone function $g_{i}, i=1$, 2 , or a vertical segment (in the latter case with an abuse of language we say that $\gamma_{i}$ coincides with the graph of a degenerate monotone function $g_{i}$ ); 
(iii) denoting by $\mathbf{a}_{1}$ and $\mathbf{a}_{2}$ the endpoints of the lower side $s_{2}$, there exists $\alpha>0$ and $0<r_{1} \leq l_{2}$ such that

$$
B\left(\mathbf{a}_{1}, r_{1}\right) \cap T \text { and } B\left(\mathbf{a}_{2}, r_{1}\right) \cap T \text { contain a sector of angle } \alpha .
$$

Note that one of the two monotone functions, say $g_{1}$, is non-decreasing while $g_{2}$ is nonincreasing. We denote by $l_{1}, l_{2}$ the length of $s_{1}, s_{2}$ respectively, and we call the distance $h$ between $s_{1}$ and $s_{2}$ the height of the generalized trapezoid $T$. Finally, let $R$ be the maximal rectangle contained in $T$ with one side coinciding with the shorter side $s_{1}$. The center $\mathbf{c}$ of such a rectangle will be called the center of the generalized trapezoid $T$.

Theorem 4.3 (Korn's Inequality III: generalized trapezoids) Let $p>1$ and let $T$ be a generalized trapezoid with center $\mathbf{c}$, height $h$, and parallel sides $s_{1}, s_{2}$ of length $l_{1}, l_{2}$ respectively (and without loss of generality $l_{1} \leq l_{2}$ ). Assume that the non-degeneracy condition (4.7) holds for some $\alpha>0$ and for some $0<r_{1} \leq l_{2}$ and let $0<m_{1}<m_{2}<+\infty$ satisfy

$$
m_{1} \leq \min \left\{\frac{l_{1}}{l_{2}}, \frac{r_{1}}{l_{2}}, \frac{h}{l_{2}}\right\} \leq \max \left\{\frac{l_{1}}{l_{2}}, \frac{r_{1}}{l_{2}}, \frac{h}{l_{2}}\right\} \leq m_{2} .
$$

Then there exists a constant $c>0$ depending only on $p, m_{1}, m_{2}$, and $\alpha$ such that

$$
\int_{T}|\nabla \mathbf{u}|^{p} d \mathbf{x} \leq c \int_{T}|\mathbf{E}(\mathbf{u})|^{p} d \mathbf{x}
$$

for all $\mathbf{u} \in W^{1, p}\left(T ; \mathbb{R}^{2}\right)$ with

$$
\int_{B}\left(\nabla \mathbf{u}-(\nabla \mathbf{u})^{T}\right) d \mathbf{x}=\mathbf{0}
$$

where $B$ is the (open) ball centered at $\mathbf{c}$ with radius $m_{1} l_{2} / 2$.

Proof. Since for every $\lambda>0$ and every vector $\mathbf{u}_{0} \in \mathbb{R}^{2}$ inequality (4.8) is invariant under the transformation

$$
\mathbf{u} \in W^{1, p}\left(T ; \mathbb{R}^{2}\right) \longrightarrow \mathbf{u} \circ \mathbf{r}_{\lambda}+\mathbf{u}_{0} \in W^{1, p}\left(\lambda T ; \mathbb{R}^{2}\right),
$$

where $\mathbf{r}_{\lambda}$ denotes the dilation map $\mathbf{x} \mapsto \mathbf{x} / \lambda$, we may assume that

$$
l_{2}=1, \quad m_{1} \leq \min \left\{l_{1}, r_{1}, h\right\} \leq \max \left\{l_{1}, r_{1}, h\right\} \leq m_{2}, \quad \operatorname{diam} T \leq \sqrt{1+m_{2}^{2}},
$$

and

$$
\int_{B} \mathbf{u} d \mathbf{x}=0
$$

We can also assume that the lower side $s_{2}$ coincides with the segment $[0,1] \times\{0\}$. We first claim that there exists a constant $c_{1}=c_{1}\left(p, m_{1}, m_{2}, \alpha\right)>0$ such that for all $\mathbf{u} \in W^{1, p}\left(T ; \mathbb{R}^{2}\right)$

$$
\int_{T}|\nabla \mathbf{u}|^{p} d \mathbf{x} \leq c_{1}\left(\int_{T}|\mathbf{u}|^{p} d \mathbf{x}+\int_{T}|\mathbf{E}(\mathbf{u})|^{p} d \mathbf{x}\right) .
$$

To this purpose observe that by (4.7) and (4.10) the isosceles trapezoid $T_{1}$ (with base angles equal to $\alpha$ and left and right sides equal to $m_{1}$ ) depicted in Figure 3 is contained in $T$. Consider also the rectangle $R_{1}=(0,1) \times\left(-m_{2}, 0\right]$ and note that any $\mathbf{u} \in W^{1, p}\left(T ; \mathbb{R}^{2}\right)$ can be extended to a function $\mathbf{w} \in W^{1, p}\left(T \cup R_{1} ; \mathbb{R}^{2}\right)$ so that

$$
\int_{R_{1}}|\mathbf{w}|^{p} d \mathbf{x} \leq c_{0} \int_{T_{1}}|\mathbf{u}|^{p} d \mathbf{x} \quad \text { and } \quad \int_{R_{1}}|\nabla \mathbf{w}|^{p} d \mathbf{x} \leq c_{0} \int_{T_{1}}|\nabla \mathbf{u}|^{p} d \mathbf{x},
$$

where the constant $c_{0}>0$ depends only on $T_{1}$, that is, on $m_{1}$ and $\alpha$. Let $\hat{\mathbf{c}}$ be the symmetric point to $\mathbf{c}$ with respect to segment $s_{2}$ and call $B^{\prime}$ the ball centered at $\hat{\mathbf{c}}$ with radius $m_{1} / 2$. Then 
it is easy to see that $T \cup R_{1}$ is starshaped with respect to $B^{\prime}$. Hence by Theorem 4.1 there exists a constant $c^{\prime}=c^{\prime}\left(p, m_{1}, m_{2}\right)$ such that

$$
\int_{T \cup R_{1}}|\nabla \mathbf{w}|^{p} d \mathbf{x} \leq c^{\prime}\left(\int_{T \cup R_{1}}|\mathbf{w}|^{p} d \mathbf{x}+\int_{T \cup R_{1}}|\mathbf{E}(\mathbf{w})|^{p} d \mathbf{x}\right) .
$$

Taking into account (4.13) we deduce from the last inequality that

$$
\int_{T}|\nabla \mathbf{u}|^{p} d \mathbf{x} \leq \int_{T \cup R_{1}}|\nabla \mathbf{w}|^{p} d \mathbf{x} \leq c^{\prime}\left(c_{0}+1\right) \int_{T}|\mathbf{u}|^{p} d \mathbf{x}+c^{\prime} \int_{T \cup R_{1}}|\mathbf{E}(\mathbf{w})|^{p} d \mathbf{x} .
$$

Finally by (4.13) and by applying Theorem 4.1 in $T_{1}$ we obtain

$$
\int_{R_{1}}|\mathbf{E}(\mathbf{w})|^{p} d \mathbf{x} \leq c_{0} \int_{T_{1}}|\nabla \mathbf{u}|^{p} d \mathbf{x} \leq c^{\prime \prime}\left(\int_{T}|\mathbf{u}|^{p} d \mathbf{x}+\int_{T}|\mathbf{E}(\mathbf{u})|^{p} d \mathbf{x}\right)
$$

for some $c^{\prime \prime}>0$ depending only on $\alpha$ and $m_{1}$. Inequality (4.12) follows now from (4.14) and (4.15)

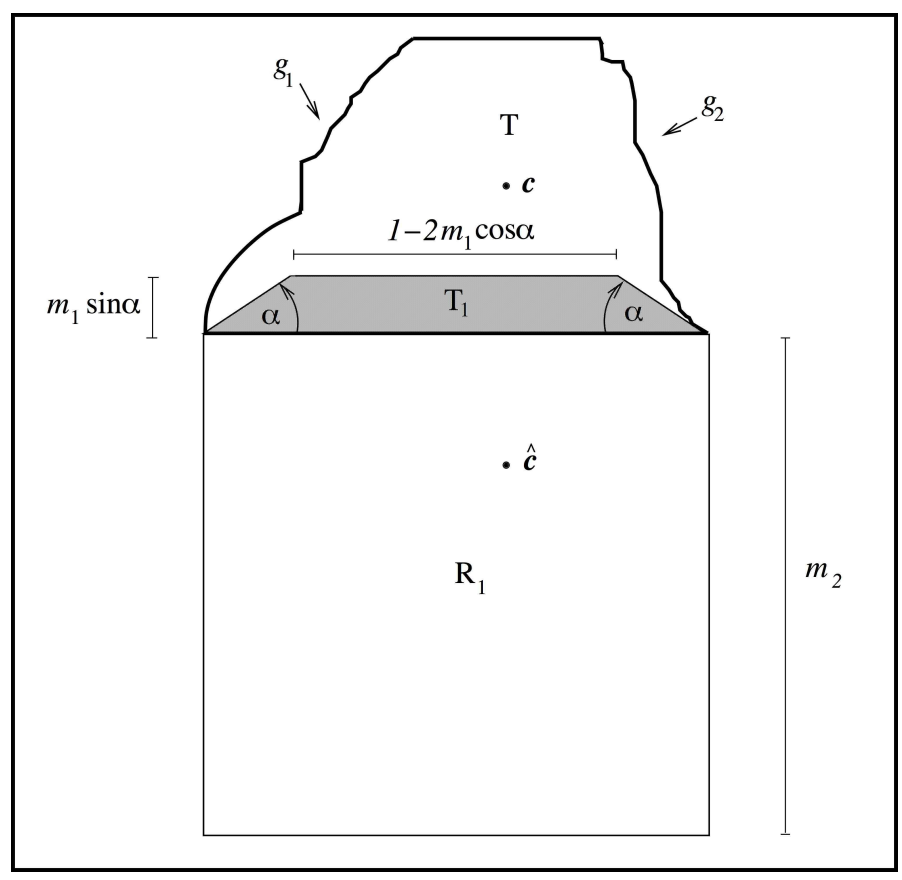

Figure 3: The sets $T_{1}$ and $R_{1}$.

As in the proof of Theorem 4.2 it is now sufficient to show the existence of a constant $c_{2}=$ $c_{2}\left(p, m_{1}, m_{2}\right)>0$ such that

$$
\int_{T}|\mathbf{u}|^{p} d \mathbf{x} \leq c_{2} \int_{T}|\mathbf{E}(\mathbf{u})|^{p} d \mathbf{x}
$$

for all $\mathbf{u} \in W^{1, p}\left(T ; \mathbb{R}^{2}\right)$ satisfying (4.9) and (4.11) . Assume by contradiction that (4.16) fails. Then there exists a sequence of generalized trapezoid $T_{n}$ as in the statement and a sequence $\mathbf{u}_{n} \in W^{1, p}\left(T_{n} ; \mathbb{R}^{2}\right)$ such that

$$
\int_{B_{n}} \mathbf{u}_{n} d \mathbf{x}=\mathbf{0}, \quad \int_{B_{n}}\left(\nabla \mathbf{u}_{n}-\left(\nabla \mathbf{u}_{n}\right)^{T}\right) d \mathbf{x}=\mathbf{0}
$$


and

$$
\int_{T_{n}}\left|\mathbf{u}_{n}\right|^{p} d \mathbf{x}=1, \quad \lim _{n \rightarrow \infty} \int_{T_{n}}\left|\mathbf{E}\left(\mathbf{u}_{n}\right)\right|^{p} d \mathbf{x}=0 .
$$

By rescaling and translating we may assume that for every $n$ the generalized trapezoid $T_{n}$ is centered at $\mathbf{c}$, satisfies (4.10), and is contained in a fixed rectangle $R_{2}$. In particular we may assume that $B_{n}=B$ for every $n$. From (4.12) and (4.17) we deduce that

$$
\sup _{n} \int_{T_{n}}\left|\nabla \mathbf{u}_{n}\right|^{p} d \mathbf{x}<+\infty .
$$

Let $g_{1, n}, g_{2, n}$ be the two monotone (possibly degenerate) functions associated with $T_{n}$. It is well known (see for instance [1]) that a rotation of $\pi / 4$ transforms the extended graph of $g_{i, n}$ into the graph of a 1-Lipschitz function (the rotation should be clockwise for the non-decreasing function $g_{1, n}$ and counterclockwise for the non-increasing function $g_{2, n}$ ). Exploiting this observation and (4.7) one can see that it is possible to extend the functions $\mathbf{u}_{n}$ to the rectangle $R_{2}$ in such a way that the resulting functions, still denoted by $\mathbf{u}_{n}$, are equibounded in $W^{1, p}\left(R_{2} ; \mathbb{R}^{2}\right)$. Using Helly's theorem for monotone functions, it is also easy to see that we can find a generalized trapezoid $T_{\infty}$, still contained in $R_{2}$, such that, up to a subsequence, $\chi_{T_{n}} \rightarrow \chi_{T_{\infty}}$ a.e.. We can now proceed exactly as in the last part of the proof of Theorem 4.2.

Remark 4.4 The final part of the previous proof shows in particular that $T$ is a Lipschitz domain and so one can extend from the very beginning the function $\mathbf{u}$ to a fixed rectangle. However, it is not possible to control the $L^{p}$ norm of the symmetrized gradient of the extended function with the $L^{p}$ norm of the symmetrized gradient of $\mathbf{u}$. This explains why we had to use a more involved argument.

A simple mollification argument shows the following result:

Corollary 4.5 Let $T$ and $B$ as in the previous theorem. Then for every $\mathbf{u} \in W_{l o c}^{1, p}\left(T ; \mathbb{R}^{2}\right)$ satisfying (4.9) and

$$
\int_{T}|\mathbf{E}(\mathbf{u})|^{p} d \mathbf{x}<+\infty
$$

it follows that $\mathbf{u} \in W^{1, p}\left(T ; \mathbb{R}^{2}\right)$ and (4.8) holds.

\section{Acknowledgment}

The authors are profoundly indebted to Robert Kohn for multiple discussions on the subject of this work and for his guidance toward relevant literature. Also, numerous fruitful conversations were carried out with P. Cermelli, A. Chambolle, M. Gurtin, V. Millot and B.J. Spencer. The authors thank the Center for Nonlinear Analysis (NSF Grant No. DMS-0405343) for its support during the preparation of this paper. The research of I. Fonseca was partially supported by the National Science Foundation under Grant No. DMS-040171 and that of G. Leoni under Grant No. DMS-0405423.

\section{References}

[1] Alberti G.; Ambrosio L., A geometrical approach to monotone functions in $R^{n}$. Math. Z. 230 (1999), 259-316.

[2] Ambrosio L.; Fusco N.; Pallara D., Functions of bounded variation and free discontinuity problems. Oxford Mathematical Monographs. The Clarendon Press, Oxford University Press, New York, 2000. 
[3] Bonnet A., On the regularity of edges in image segmentation. Ann. Inst. H. Poincaré Anal. Non Linéaire 13 (1996), 485-528.

[4] Bonnetier E..; Chambolle A., Computing the equilibrium configuration of epitaxially strained crystalline films. SIAM J. Appl. Math. 62 (2002), 1093-1121.

[5] Braides A., Г-Convergence for beginners, Oxford University Press, 2002.

[6] Braides A.; Chambolle A.; Solci M., A relaxation result for energies defined on pairs set-function and applications, preprint.

[7] Chambolle A.; Larsen C. J., $C^{\infty}$ regularity of the free boundary for a two-dimensional optimal compliance problem. Calc. Var. Partial Differential Equations 18 (2003), 77-94.

[8] Chambolle A.; Solci M., Interaction of a bulk and a surface energy with a geometrical constraint, preprint.

[9] Chen K. M.; Jesson D. E.; Pennycook S. J.; Thundat T.; Warmack R. J., Cuspidal pit formation during the growth of $S i_{x} G e_{1-x}$ strained films. Applied Physics Letters 66 (1995), 34-36.

[10] Chiu, C.-H.; GaO, H. Stress Singularity along a cycloid rough surface. Int. J. Solids Structures 30 (1993), 2983-3012.

[11] Dal Maso G., An Introduction to $\Gamma$-Convergence, Birkhaüser, 1993.

[12] DiBenedetto E., Real analysis. Birkhäuser Advanced Texts: Basler Lehrbücher. Birkhäuser Boston, Inc., Boston, MA, 2002.

[13] Federer H., Geometric measure theory, Springer, Berlin, 1969.

[14] Gao H.; Nix W.D., Surface Roughening of Heteroepitaxial Thin Films. Annual Review of Materials Science, 29 (1999), 173-209.

[15] Giaquinta M, Multiple integrals in the Calculus of Variations and Nonlinear Elliptic Systems. Annals of Mathematics Studies, 105. Princeton University Press, Princeton, NJ, 1983.

[16] Grinfeld M. A., Stress driven instabilities in crystals: mathematical models and physical manifestation, J. Nonlinear Sci. 3 (1993), 35-83.

[17] Grisvard P., Elliptic problems in nonsmooth domains. Monographs and Studies in Mathematics, 24. Pitman (Advanced Publishing Program), Boston, MA, 1985.

[18] Grisvard P., Singularités en elasticité. Arch. Rational Mech. Anal. 107 (1989), 157-180.

[19] Koch H.; Leoni G.; Morini M., On Optimal regularity of Free Boundary Problems and a Conjecture of De Giorgi. Comm. Pure Applied Math. 58 (2005), 1051-1076.

[20] Kukta R.V.; Freund L.B., Minimum energy configurations of epitaxial material clusters on a lattice-mismathched substrate. J. Mech. Phys. Solids 45 (1997), 1835-1860.

[21] LeOni G.; Morini M., Necessary and sufficient conditions for the chain rule in $W_{\text {loc }}^{1,1}\left(\mathbb{R}^{N} ; \mathbb{R}^{d}\right)$ and $B V_{\text {loc }}\left(\mathbb{R}^{N} ; \mathbb{R}^{d}\right)$, to appear on J. Eur. Math. Soc.

[22] Lieb E. H.; Loss M., Analysis. Second edition. Graduate Studies in Mathematics, 14. American Mathematical Society, Providence, RI, 2001.

[23] Mosolov P. P.; Muasnikov V. P., A proof of Korn's inequality. (Russian) Dokl. Akad. Nauk SSSR 201 (1971), 36-39. 
[24] NeČAs J.; HlaváČEK I., Mathematical theory of elastic and elasto-plastic bodies: an introduction. Studies in Applied Mechanics, 3. Elsevier Scientific Publishing Co., Amsterdam-New York, 1980.

[25] NiCAise S., About the Lamé system in a polygonal or a polyhedral domain and a coupled problem between the Lamé system and the plate equation. I. Regularity of the solutions. Ann. Scuola Norm. Sup. Pisa Cl. Sci. (4) 19 (1992), 327-361.

[26] Spencer B. J., Asymptotic derivation of the glued-wetting-layer model and contact-angle condition for Stranski-Krastanow islands. Physical Review B 59 (1999), no. 3, 2011-2017.

[27] Spencer B. J.; Meiron D.I., Nonlinear Evolution of the Stress-Driven Morphological Instability in a Two-Dimensional Semi-Infinite Solid. Acta Metallurgica et Materialia 42 (1994), 3629-3641.

[28] Spencer B. J.; Tersoff J., Equilibrium shapes and properties of epitaxially strained islands. Physical Review Letters 79 (1997), 4858-4861. 